\title{
Small GTPases
}

\author{
David J. Reiner ${ }^{1,2 \S}$ and Erik A. Lundquist ${ }^{3 \S}$ \\ ${ }^{1}$ Institute of Biosciences and Technology, Texas A\&M Health Science Center, Houston, TX USA \\ ${ }^{2}$ Department of Medical Physiology, College of Medicine, Texas A\&M University Health Science \\ Center, Temple, TX USA \\ ${ }^{3}$ Department of Molecular Biosciences, University of Kansas, Lawrence, KS USA
}

\section{Table of Contents}

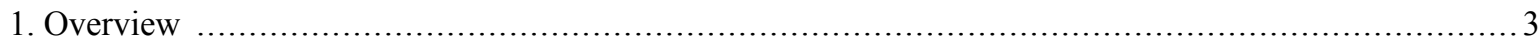

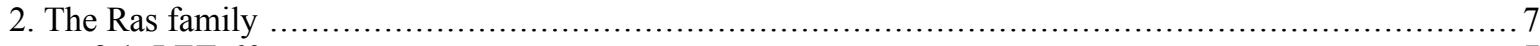

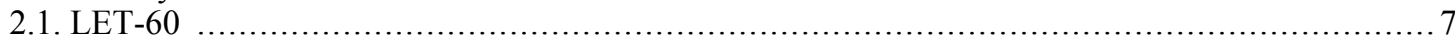

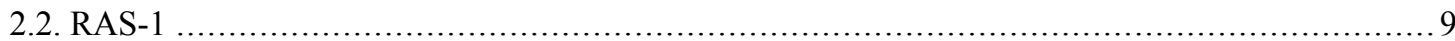

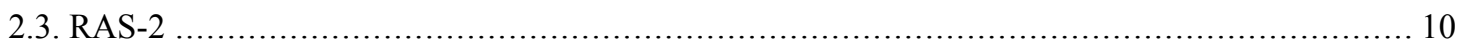

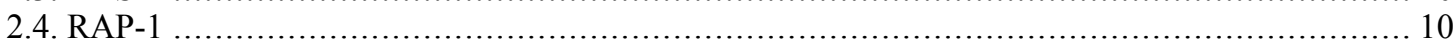

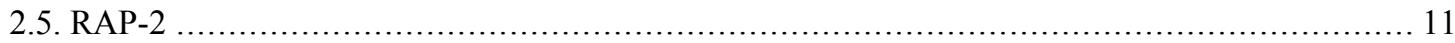

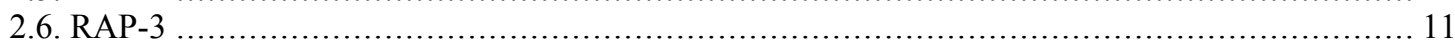

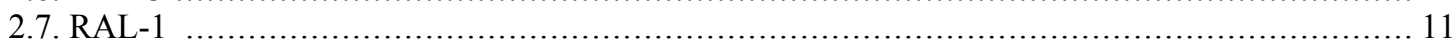

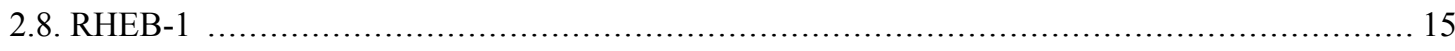

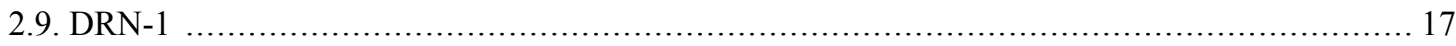

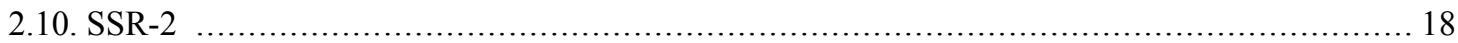

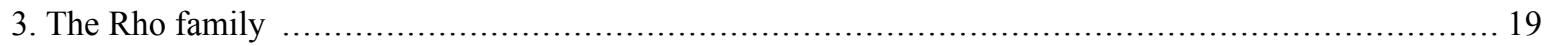

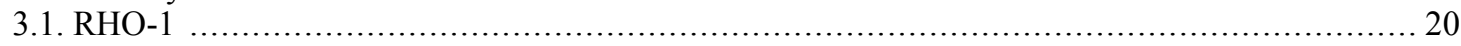

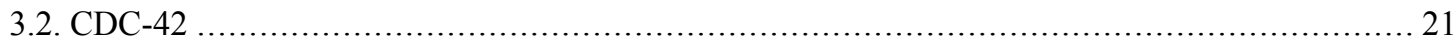

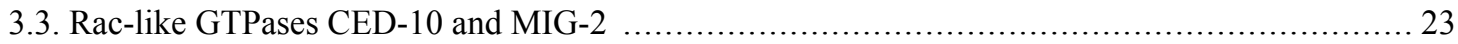

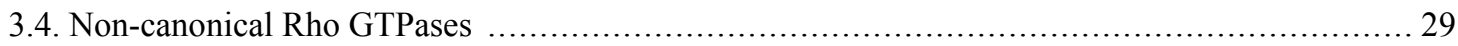

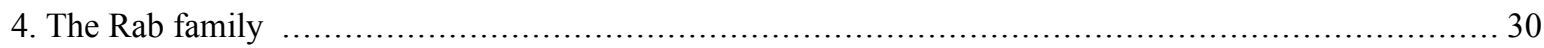

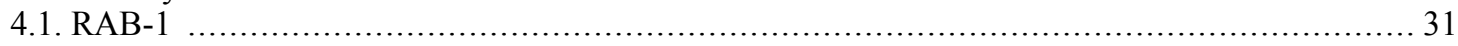

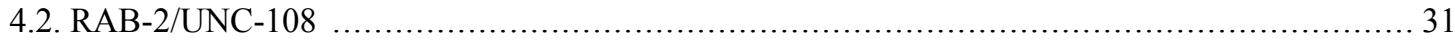

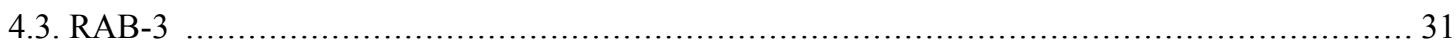

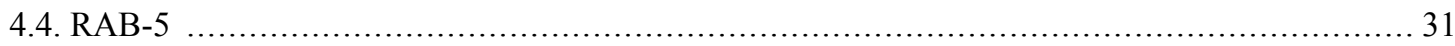

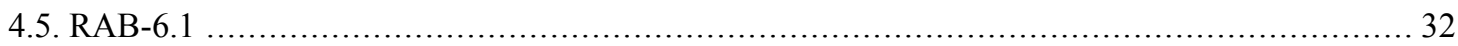

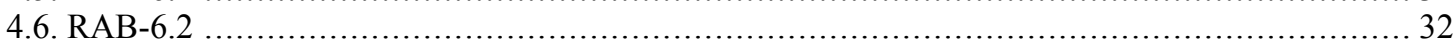

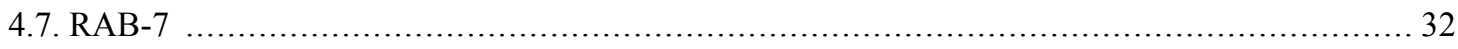

*Edited by Iva Greenwald. Last revised February 23, 2016. Published in its final form August 16, 2018. This chapter should be cited as: Reiner D. J., Lundquist E. A. Small GTPases. (August 16, 2018), WormBook, ed. The C. elegans Research Community, WormBook, doi/10.1895/wormbook.1.67.2, http://www.wormbook.org.

Copyright: (C) 2016 David J. Reiner and Erik A. Lundquist. This is an open-access article distributed under the terms of the Creative Commons Attribution License, which permits unrestricted use, distribution, and reproduction in any medium, provided the original author and source are credited.

${ }^{\S}$ To whom correspondence should be addressed. Email: dreiner@ibt.tamhsc.edu or erikl@ku.edu 


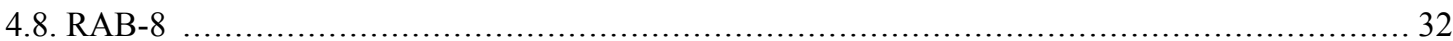

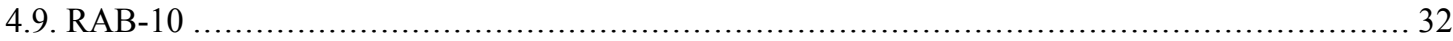

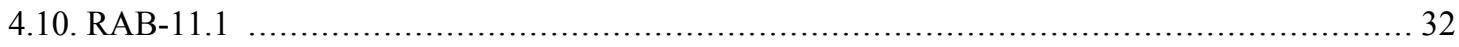

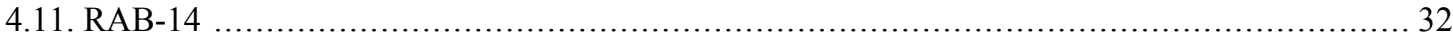

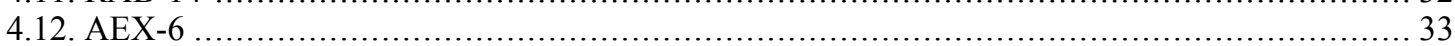

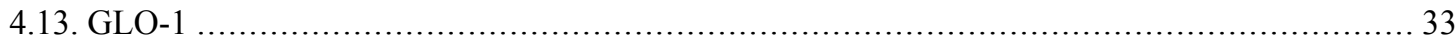

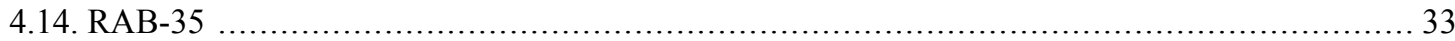

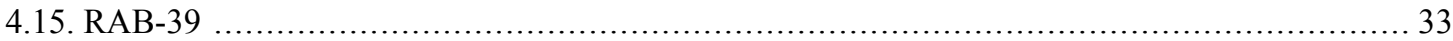

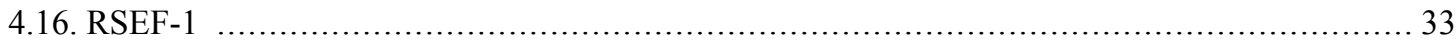

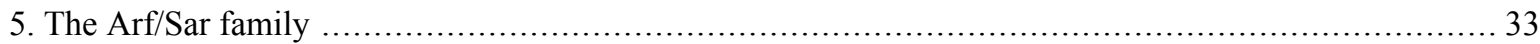

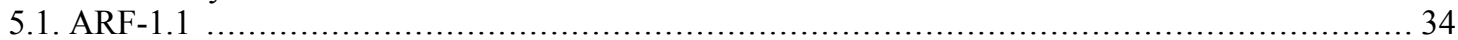

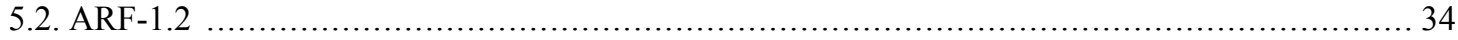

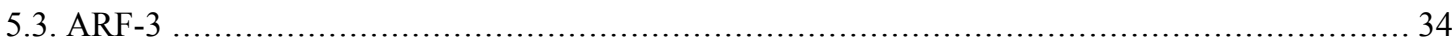

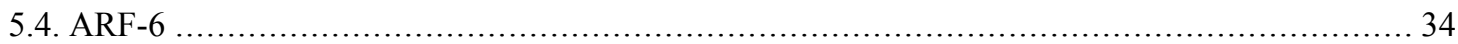

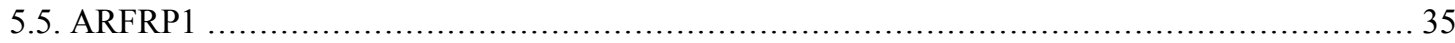

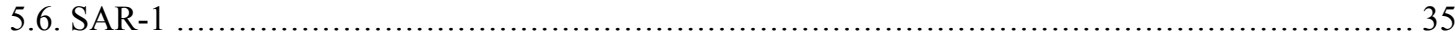

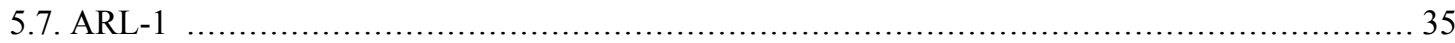

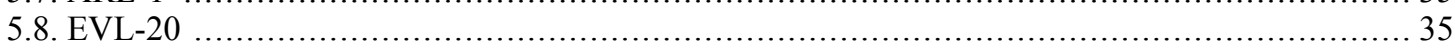

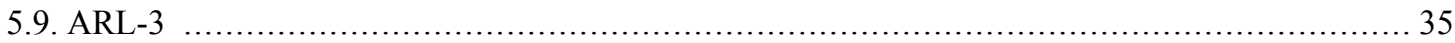

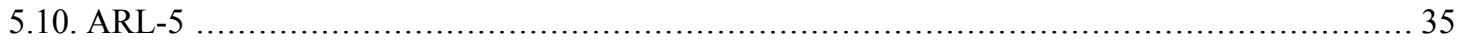

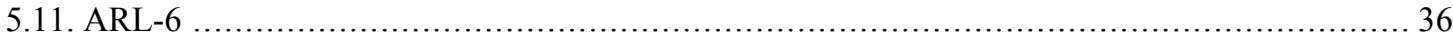

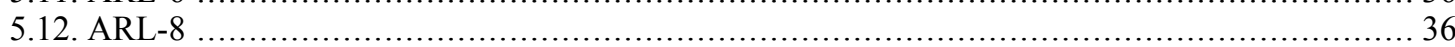

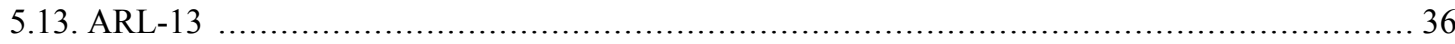

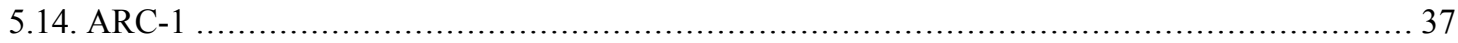

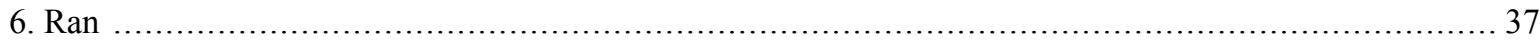

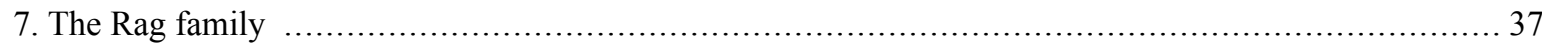

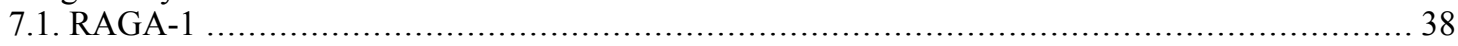

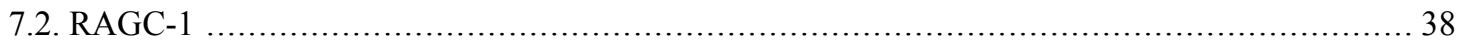

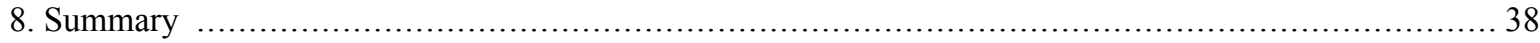

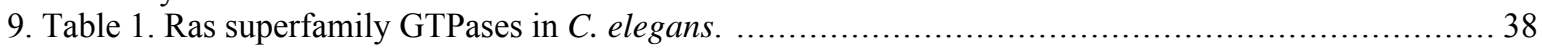

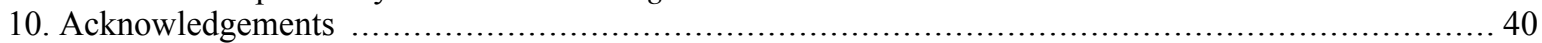

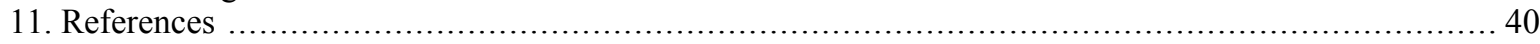

\begin{abstract}
Members of the protein superfamily of small guanosine triphosphatases, also known as small GTPases, small G-proteins, or the Ras superfamily, are involved in nearly every aspect of cell biology. Small GTPases are tightly regulated molecular switches that make binary on/off decisions through controlled loading of GTP (activation) and hydrolysis of GTP to GDP (inactivation). Small GTPases typically function as nodal points that integrate broad upstream regulatory inputs and disseminate broad effector outputs. The superfamily comprises five families that are conserved across eukaryotes: Ras, Rho, Rab, Arf, and Ran. Each family, besides Ran, has radiated functionally since our last common ancestor with fungi, and certain subfamilies persist throughout metazoa. The double genome duplication leading to vertebrates resulted in two to four genes for many subfamilies, plus some novel mammalian additions. Here we discuss general principles of small GTPase biology, survey the $C$. elegans complement of small GTPases and how they compare to their mammalian counterparts, and note atypical nematode members that do not fall into discrete subfamilies. We do not discuss the multitude of other proteins with catalytic guanosine triphosphatase domains that fall outside the small GTPase/Ras superfamily.
\end{abstract}




\section{Overview}

The Ras (Rat sarcoma) superfamily of small GTPases is an ancient group of molecular switches whose functions have radiated phylogenetically to encompass broad areas of cell biology. The founding members of the superfamily are the Ras proto-oncogenes, discovered in the early 1980s, which are conserved from yeast to $C$. elegans, Drosophila, and mammals. Ras itself, however, composes a tiny part of the overall signaling capabilities of the small GTPase superfamily. Members of the superfamily are key regulators of most processes in the cell, including differentiation, proliferation, vesicle and organelle dynamics and transport, nuclear dynamics, and regulation of the cytoskeleton. What they share in common is the GDP/GTP switch or, in some cases, just the structure of the GTPase domain, even if catalytically inactive.

In addition to being the founding member of the superfamily, Ras is also the reference protein of the Ras family of small GTPases - one of five families in the Ras superfamily: the others being Rho (Ras homology), Arf (ADP-ribosylation factor), Rab (Ras-like in brain), and Ran (Ras-like nuclear). (Figure 1). Ras family members generally participate in signal transduction, though, like many superfamily members, they perform functions outside of their stereotypical roles. Rho family members typically regulate cytoskeletal dynamics. The Rab family members generally control fusion and trafficking of vesicles and larger bodies. Functions of Arf family members partially overlap with those of Rabs, and are generally involved in vesicle biogenesis and recycling, intracellular trafficking, and cytoskeletal regulation. The single Ran controls nuclear translocation and structure.

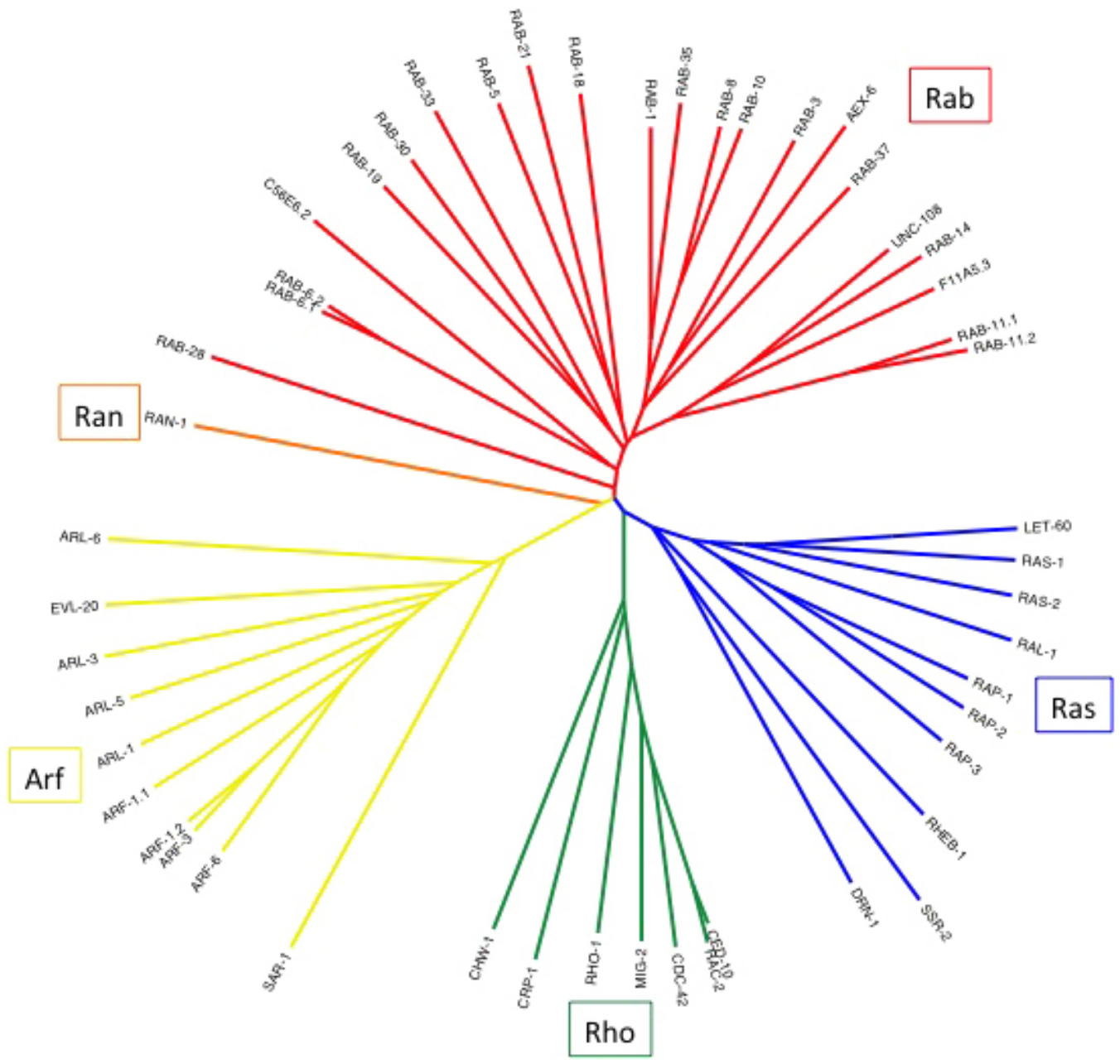

Figure 1. A dendrogram of the $\boldsymbol{C}$. elegans small GTPases. The Ras superfamily consists of the Ras, Rho, Rab, and Arf families, with Ran comprising its family of one. The core GTPase domain is the unifying feature of this group, while N-and C-terminal extensions are much less conserved and could distort alignments. Thus only the core GTPase domain was used for the alignment and generation of this rooted tree (via Clustal X, with trees generated in FigTree). 
Small GTPases cycle between two basic states: inactive GDP-bound (Ras•GDP), and active, GTP-bound (Ras•GTP) (Figure 2). Two major classes of regulators control the GTP binding state of small GTPases. Guanine nucleotide exchange factors (GEFs) eject GDP from Ras•GDP, and the much higher levels of free GTP than GDP in the cell favor GTP loading to produce Ras•GTP. The catalytic activity of intrinsic GTP hydrolysis is typically poor. But GTPase activating proteins (GAPs) stimulate GTP hydrolysis to convert the protein from Ras $\bullet$ GTP to Ras $\bullet$ GDP.

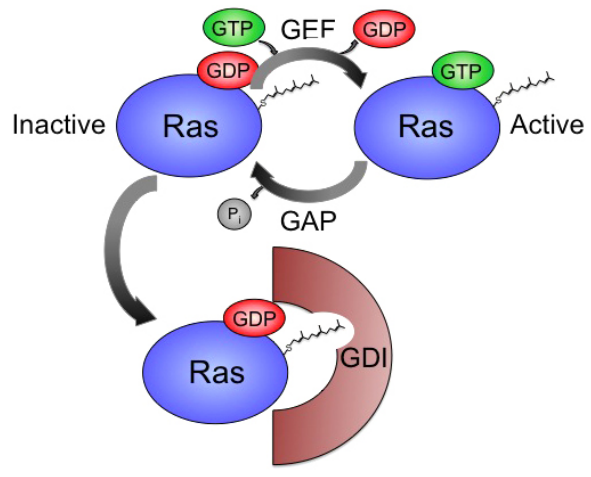

Figure 2. The small GTPase cycle. GEFs promote GTP loading to activate small GTPases, while GAPs stimulate intrinsic GTP hydrolysis to inactivate small GTPases. Guanine nucleotide dissociation inhibitors (GDIs) sequester GDP-bound, prenylated GTPases, and can sequester or chaperone them among plasma membrane and subcellular compartments.

GEFs and GAPs are multi-domain proteins that integrate diverse arrays of cellular inputs to confer precise spatiotemporal control of small GTPase activation (Bos et al., 2007). GEF catalytic domains vary widely; canonical types can be identified by homology, typically within GTPase families (e.g., S. cerevisiae CDC25 domains for the Ras family, DH-PH and DOCK domains for the Rho family, and DENN domains for the Rab family). Additionally, non-canonical GEFs are still being identified based on function rather than homology (Bos et al., 2007). GEF and GAP domains are typically unconserved between GTPase families. Distinctive GAP domains are specific to each GTPase family: RasGAP, RhoGAP, and TBC domains are diagnostic for the Ras, Rho, and Rab families, respectively (Barr and Lambright, 2010). GEFs and GAPs can be found with a wide diversity of accompanying domains, and it is thought that different GEFs and GAPs orchestrate activity of the same small GTPase in different tissues or in different subcellular compartments of the same tissue type. For example, 69 different mammalian DH-PH RhoGEFs and 11 DOCK-ELMO RhoGEFs have been identified to regulate 22 Rho family members (Rossman et al., 2005). The diversity of inputs to GEFs and GAPs encompasses all of cell biology- from direct binding by signaling proteins like heterotrimeric G-proteins, to second messengers like cAMP, to structural proteins (Bos et al., 2007).

The common structural and biochemical feature of the small GTPase superfamily is the approximately 170 residue $\mathrm{G}$ domain, which governs nucleotide binding, GTP hydrolysis, and effector interactions. The GTPase domain is defined by five G-boxes with certain structurally conserved residues across the superfamily: G1 (GxxxxGKS/T), G2 (T), G3 (DxxGQ/H/T), G4 (T/NKxD), and G5 (C/SAK/L/T) (Wennerberg et al., 2005) (Figure 3). Effector binding is governed by the Switch I and Switch II regions of the GTPase, which undergo a conformation change upon GTP binding, particularly the core effector-binding region in Switch I, around the G2 box (Holbrook and Kim, 1989). Additional N- and C-terminal extensions further regulate function, mostly through membrane association in defined subcellular compartments, typically the plasma membrane but extending to most organelles and vesicles.

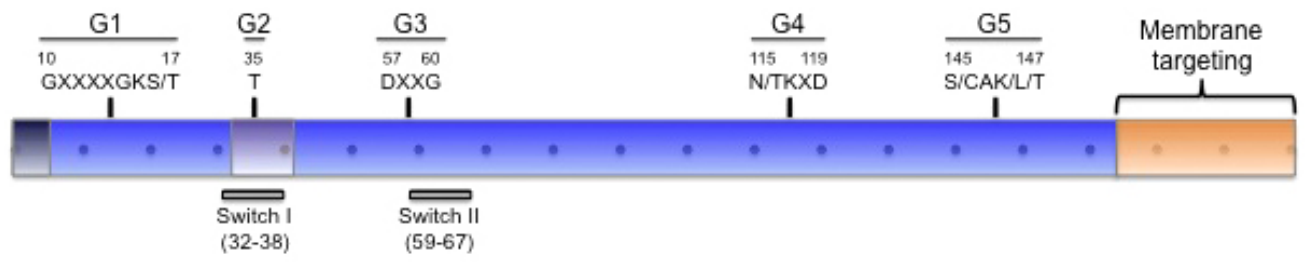

Figure 3. Functional domains of LET-60/Ras inferred from mammalian Ras. Blue indicates the G domain (also known as the GTPase domain; residues 5-166 in LET-60 and mammalian H/N/K-Ras), purple indicates the core effector-binding region that mediates direct interaction with effectors, and orange indicates the C-terminal hypervariable region and CAAX (HVR + CAAX) that is typically lipid modified and governs membrane localization. The five $\mathrm{G}$ motifs (also called $\mathrm{G}$ boxes) and consensus sequences among small GTPases are shown above the diagram with canonical $C$. elegans LET-60 and mammalian H/N/K-Ras numbering. Switch I and Switch II are regions whose conformation changes with the change from GDP loading (inactive) to GTP loading (active) states. Each dot represents 10 amino acids. This figure was adapted from (Wennerberg et al., 2005). 
An ironic feature of small GTPases, which frequently confuses newcomers, is that the enzymatic function of small GTPases - GTP hydrolysis to generate GDP and free inorganic phosphate-inactivates the protein. Thus selectively altering the catalytic function of a small GTPase without disrupting protein structure locks the protein in the "on" state, while promoting enzymatic function hastens inactivation. Disruption of protein structure with strong loss-of-function or null mutations abrogates signaling.

The highly conserved structure of the Ras superfamily G domain has allowed the characterization and broad application of genetic tools developed through biochemical studies and mostly validated in mammalian cell-based assays (Wennerberg et al., 2005). Based on mutations found in oncogenic Ras (and following Ras numbering), substitutions at positions 12, 13, and 61 result in constitutive activity: canonical mutations are G12V, G13E, and Q61L, with G13E causing only moderate activation. S17N causes a dominant-negative GTPase; this reagent should be used with caution, since the S17N GTPase sequesters interacting GEFs, thus blocking their activation of shared substrates and potentially yielding misleading results. The F28L or "fast-cycling" mutation confers a GEF-insensitive but GAP-sensitive gain of function, which is particularly useful in generating intermediate-level activation, or for GTPases where GDP-GTP cycling is more important than the actual nucleotide-bound state (Reinstein et al., 1991). Variations in applicability occur across the superfamily, so utilization of these tools for each of its mammalian ortholog(s) should be assessed before applying them to a $C$. elegans protein for the first time.

Most but not all Ras superfamily small GTPases are targeted to specific subcellular compartments by membrane-targeting sequences and lipid modification. Ras, Rho, and Rab family members are generally C-terminally modified via farnesyl, geranylgeranyl, or palmitoyl lipids covalently linked to C-terminal cysteines, followed by cleavage of the residues after the modified cysteine (Wennerberg and Der, 2004) (Figure 4). A myristoyl group generally N-terminally modifies Arf family members, and Ran is not modified. For unknown reasons, certain subfamilies in each family are unmodified. Where present, the lipid modification inserts into the membrane bilayer, tethering the small GTPase to the membrane. The typically strong subcellular localization of each small GTPase is collectively dictated by specific small GTPase lipid modification, composition of local membranes, C-terminal electrostatic properties (including post-translational modifications like phosphorylation), and trafficking by chaperone proteins. For most modified small GTPases the lipid modification is essential for targeting and activity. For example, Cys to Ser mutation of the C-terminal CAAX sequence to SAAX results in a non-functional protein, a good control for ectopic expression studies (as is the wild-type GTPase, which due to tight regulatory control is usually inactive when ectopically expressed). In the CRISPR-Cas9 genome-editing era, the SAAX mutation is a good tool to eliminate small GTPase function without disrupting gene structure and attendant stoichiometry of regulatory factors. The utility of this approach, as well as engineered catalytic site mutations for other proteins, is becoming evident as we learn more about the impact of non-canonical regulatory networks, particularly those of non-coding RNAs (Tay et al., 2014).

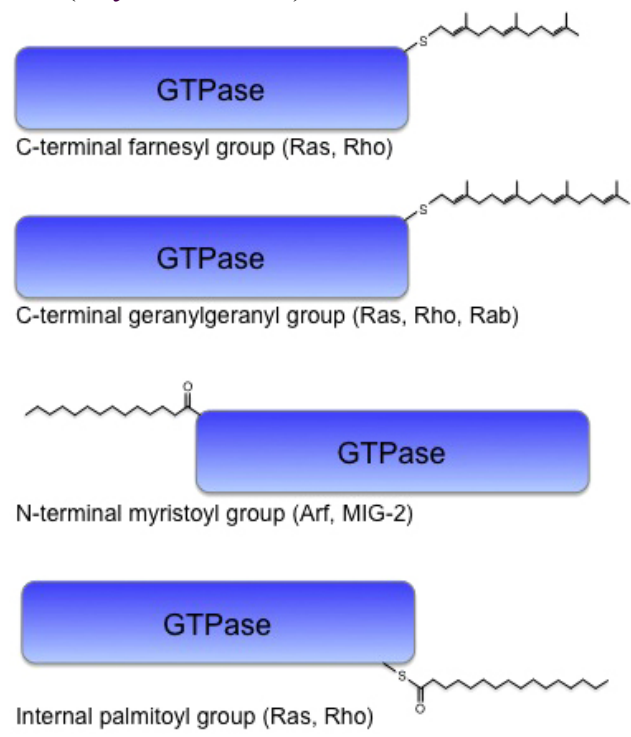

Figure 4. Lipid modifications of the Ras superfamily. Many Ras superfamily small GTPases across species are lipid modified. These examples are taken from mammalian benchmarks, but also include possible myristoylation of MIG-2. This figure is an adaptation of (Wennerberg et al., 2005). 
Yeast Ras is modified at the C-terminus by farnesyl isoprenoid lipid at the CAAX sequence and this observation was validated in mammalian cells (Casey et al., 1989; Jackson et al., 1990). This feature of Ras biology suggested that treatment with farnesyltransferase inhibitors (FTIs) could block oncogenic Ras signaling. This approach was defeated by two circumstances: first, there are many farnesylated proteins in the mammalian genome, and thus FTIs have substantial and unavoidable off-target effects; and second, when K-Ras4B farnesylation was blocked the protein was alternatively geranylgeranylated, thus bypassing blockade (Lerner et al., 1997; Sun et al., 1998). Otherwise, Ras and members of its superfamily are considered to be "undruggable," though certain highly specific covalent modifications have been successful (Ostrem et al., 2013).

Of the Ras superfamily members in C. elegans, only LET-60/Ras has been tested for lipid transferase inhibitor function: addition of the classic farnesyltransferase inhibitor (FTI) was shown to suppress the let-60 gain-of-function multivulva phenotype, consistent with inhibition of LET-60 membrane targeting (Hara and Han, 1995). HMG-CoA reductase inhibitors lovastatin and fluvastatin can generally block protein prenylation. While not validated with LET-60, these more general small molecule inhibitors should inhibit membrane localization of small GTPases, and have been used in C. elegans to guide dosing (Morck et al., 2009).

The C-terminal extension of the small GTPase after the G domain is called the "hyper-variable region" because it is poorly conserved even among paralogs in the same subfamily, though there may be conservation of total charge, post-translational sites, and of course the CAAX motif at the very C-terminus, which together influence protein function and signaling outcomes. In addition to lipid modification of the CAAX sequence targeting the protein, the hyper-variable region also contributes to subcellular localization through electrostatic charges that better fit the lipid composition of certain subcellular compartments (Cox et al., 2015). This phenomenon was most dramatically illustrated for the oncogenic human K-Ras4B splice variant, which has a lysine-rich and hence positively charged hyper-variable region. Compared to the alternatively spliced K-Ras4A or other Ras genes N-Ras and H-Ras, which have less basic hyper-variable regions, K-Ras4B plasma membrane targeting is altered and oncogenic potential is much greater (Hancock, 2003). C-terminal electrostatic charge is important enough to K-Ras4B activity that it is thought to explain the enigmatic role of plasma membrane voltage, and attendant mis-regulation of ion channels, in targeting of K-Ras to lipid rafts containing elevated phospholipids (phosphatidylserine and phosphatidylinositol 4,5-bisphosphate) (Zhou et al., 2015). Additionally, each phosphorylation event of a hyper-variable region introduces two negative charges, and alters the electrostatic charge and hence targeting to membrane compartments. The phenomenon was demonstrated in mammalian cells by PKC phosphorylation of K-Ras (Bivona et al., 2006), Aurora A phosphorylation and PP2A dephosphorylation of RalA in the Ras family (Wu et al., 2005; Sablina et al., 2007), and PKA phosphorylation of RhoA in the Rho family (Ellerbroek et al., 2003). In each of these cases the phosphorylation events physically re-targeted the small GTPase and also altered its functional output by altering encounters with effectors. Thus C-terminal phosphorylation may be a common mechanism for dynamically switching small GTPase output.

Another mechanism of small GTPase regulation is that of the chaperone, typically termed a guanine nucleotide dissociation inhibitor (GDI) (Schmick et al., 2015). GDIs have affinity for the GDP-bound, inactive form of the lipid-modified GTPase and thus can shield the hydrophobic lipid-modified tail of the small GTPase from the hydrophilic cytosol during travel between membrane compartments. The mechanisms of GDI action have been investigated for Rab, Rho, and Ras family GDIs (Garcia-Mata et al., 2011; Chandra et al., 2012; Wandinger-Ness and Zerial, 2014). This subject has not been investigated in C. elegans beyond the biochemical properties of the single RHI-1/RhoGDI (Yap et al., 1999). RHI-1 promotes increased lifespan of animals (Ha et al., 2006). $r h i-1(R N A i)$ in an RNAi-hypersensitive background confers locomotion defects (Simmer et al., 2003) as expected for general deregulation of Rho family members.

Small GTPase output is controlled through conformational shift of the Switch I and Switch II regions upon GTP binding (Colicelli, 2004). Switch I includes the effector-binding domain, a nine-residue core binding sequence that is frequently absolutely conserved between metazoan orthologs. This is not the sole sequence that dictates effector partners, since other sequences across the surface of the protein can influence stability of the GTPase-effector interface. But the region mediates activity-dependent effector interactions across the superfamily, and reasonably selective effector-binding mutations can be found within the core effector-binding domains that do not otherwise disrupt GTPase function. In many cases the different effectors have been identified, but the mechanisms by which the small GTPase switches effectors, or how multiple effectors with different functions are orchestrated, are poorly understood. In effectors the domains that mediate GTPase binding are substantially less conserved across species than are the GTPases that bind them. Thus, from an evolutionary perspective the exceptionally high conservation of effector-binding domains may reflect the use of multiple effectors. One GTPase 
and one effector could theoretically evolve together, resulting in substantial changes in sequence. But if a GTPase needs to interact with multiple binding partners, its effector binding sequence is much more constrained evolutionarily.

\section{The Ras family}

Since Ras oncoproteins are the most frequently mutated oncoproteins, with approximately $35 \%$ of all cancers harboring activating Ras mutations (Bos, 1989), Ras itself is an exceptionally well-studied protein. Additionally, Ras is the founding member of the Ras small GTPase superfamily, and all superfamily members have the fundamental Ras small GTPase architecture. Because of its status as a pioneer, many of the general mechanistic principles of small GTPase superfamily members were initially established in studies of Ras across eukaryotes from yeast to humans, including $C$. elegans. In this section, however, we focus just on immediate members of the Ras family. Ras family members share the trait that most of them function in some aspect of signal transduction. Core effector binding domains are probably the main determinants of binding partners, and are generally well conserved phylogenetically (Figure 5).

$\begin{array}{ll}\text { Rap1A } & \text { QGIFVEKYDPTIEDSYRKQVEVD-CQ } \\ \text { Rap1B } & \text { QGIFVEKYDPTIEDSYRKQVEVD-AQ } \\ \text { RAP-1 } & \text { QGIFVEKYDPTIEDSYRKQVEVD-GQ } \\ \text { Rap2A } & \text { TGTFIEKYDPTIEDFYRKEIEVD-SS } \\ \text { Rap2B } & \text { TGSFIEKYDPTIEDFYRKEIEVD-SS } \\ \text { RAP-2 } & \text { SSTFIEKYDPTIEDFYRKEIEVD-GQ } \\ \text { RAP-3 } & \text { QGIFVHTYDATIEDSYRKLSKVD-AE } \\ \text { M-RaS } & \text { QKIFVPDYDPTIEDSYLKHTEID-NQ } \\ \text { RAS-2 } & \text { QKQFVDYYDPTIEDOYIQHCEID-GN } \\ \text { R-Ras } & \text { QSYFVSDYDPTIEDSYTKICSVD-GI } \\ \text { R-Ras3 } & \text { QSYFVTDYDPTIEDSYTKQCVID-DR } \\ \text { RAS-1 } & \text { QRYFVQDYDPTIEDSYTKQCFVD-ED } \\ \text { RalA } & \text { YDEFVEDYEPTKADSYRKKVVLD-GE } \\ \text { RalB } & \text { YDEFVEDYEPTKADSYRKKVVLD-GE } \\ \text { RAL-1 } & \text { YDEFVEEYEPTKADSYRKKVVLD-GE } \\ \text { N-RaS } & \text { QNHFVDEYDPTIEDSYRKQVVID-GE } \\ \text { H-Ras } & \text { QNHFVDEYDPTIEDSYRKQVVID-GE } \\ \text { K-RaS } & \text { QNHFVDEYDPTIEDSYRKQVVID-GE } \\ \text { LET-60 } & \text { QNHFVEEYDPTIEDSYRKQVVID-GE } \\ \text { Rheb1 } & \text { EGEFSEGYDPTVETYSKIVTLG-KD } \\ \text { Rheb2 } & \text { EGQFVDSYDPTIENTFTKLITVN-GQ } \\ \text { RHEB-1 } & \text { QNIFPERYESTIEDOHSKHIAAF-HR } \\ \text { Di-Ras1 } & \text { KGTFRDTYIPTIEDTYRQVISCD-KS } \\ \text { Di-Ras2 } & \text { KGTFRESYIPTVEDTYRQVISCD-KS } \\ \text { Di-Ras3 } & \text { SGNFRHEYLPTIENTYCQLLGCS-HG } \\ \text { DRN-1 } & \text { KGTFNENYVPTIEDTYRQVISCNQKN }\end{array}$

Figure 5. Effector-binding region of human vs. C. elegans Ras family members. The entire effector-binding region of human and C. elegans proteins was aligned. The core sequence, thought to govern direct protein-protein interactions, is underlined. Core residues not conserved in the subfamily are bolded and blue, and the DRN-1 insertion is bolded and red. Human orthologs are listed above C. elegans orthologs. C. elegans protein names have a dash and number. Some human proteins have alternate names (see Table 1, Section 9).

\subsection{LET-60}

let-60 encodes the single $C$. elegans ortholog of mammalian H-, N- and K-Ras. The lysine-rich (and hence very basic) C-terminal hyper-variable region of LET-60 is most similar to K-Ras4B, the most oncogenic mammalian splice variant. The name derives from the lethality conferred by loss-of-function mutations.

The best-studied C. elegans function of LET-60/Ras is in transducing the EGF signal that patterns the vulval precursor cells (VPCs): many missense alleles were isolated based on defective patterning of the vulva (Ferguson et al., 1987). A signal originating in the anchor cell (AC) induces vulval fate specification (see WormBook chapter Vulval Development). The signal consists of EGF, which signals through the LET-23 EGF receptor (EGFR) (Aroian et al., 1990). The resulting signaling cascade is conserved throughout metazoans (Egan and Weinberg, 1993). A key link between upstream growth factor receptors like EGFR and downstream Ras was discovery of the SEM-5/Grb2 SH3-SH2-SH3 receptor tyrosine kinase (RTK) adaptor (Clark et al., 1992; Lowenstein et al., 1992; Pawson, 1992; 
Rozakis-Adcock et al., 1992). SEM-5/Grb2 in turn recruits the Sos/SOS-1 RasGEF to the RTK signaling complex (Bonfini et al., 1992; Chardin et al., 1993; Chen et al., 1997; Chang et al., 2000). Both activating and temperature-sensitive alleles of sos- 1 have been characterized (Rocheleau et al., 2002; Modzelewska et al., 2007).

LET-60/Ras is necessary and sufficient for vulval induction (Beitel et al., 1990; Han et al., 1990; Han and Sternberg, 1990). Reduced LET-60 signaling causes an under-induced vulvaless (Vul) phenotype, while gain of LET-60 function causes $1^{\circ}$ hyper-induction and multivulva (Muv) animals (Figure 6). Three putative RasGAPs have been identified, and GAP-1 and GAP-3 function redundantly to repress LET-60 activity during vulval development, while different combinations of GAPs regulate LET-60-dependent functions in other tissues (Hajnal et al., 1997; Stetak et al., 2008).

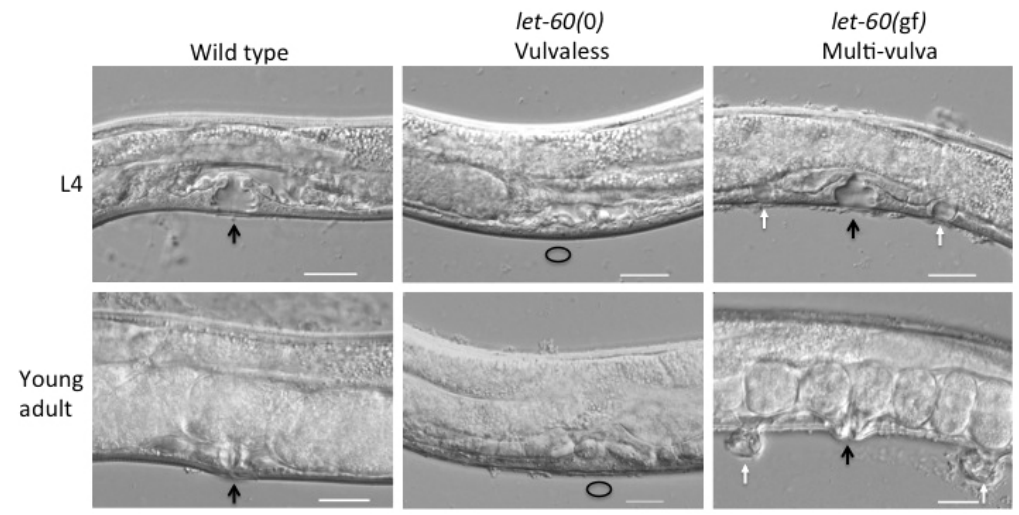

Figure 6. LET-60 functions as a binary switch controlling vulval induction. Vulval development is shown at the late L4 (top row) and adult (bottom row) stages. LET- 60 activated by EGF and EGFR induces a single $1^{\circ}$ cell flanked by two $2^{\circ} \mathrm{s}$ cells, which divide through stereotyped lineages and undergo morphogenesis to form the L4 "Christmas tree" invagination (above) and mature vulva (below). Black arrows indicate normal vulvae, white arrows indicate ectopic pseudovulvae, and circles represent missing vulvae. The let-60(dx16) null deletion allele confers a $100 \%$ vulvaless phenotype (middle) (Gumienny et al., 1999). The let-60(n1046gf) G13E moderately activating allele (right) induces ectopic $1^{\circ}$ cells, which go on to form ectopic pseudovulvae (scale bar $=20 \mu \mathrm{m}$ ). Image credit: Neal Rasmussen from the Reiner lab.

Most identified functions of LET-60 are associated with the LIN-45/Raf Ser/Thr kinase (Han et al., 1993), which, at around the same time, was defined as the central Ras effector in other systems, such as Drosophila R7 photoreceptor specification (Ambrosio et al., 1989) and mouse fibroblast oncogenic transformation, activating a MEK-ERK MAP kinase signaling cascade to regulate transcription (Kyriakis et al., 1992; Moodie and Wolfman, 1994).

LET-60 activation of LIN-45/Raf also plays a central role in sex myoblast migration (Sundaram et al., 1996), specification of the excretory duct cell (Yochem et al., 1997), P12 neuroectoblast induction (Jiang and Sternberg, 1998), excretory/fluid homeostasis (Schutzman et al., 2001), and olfaction (Hirotsu et al., 2000). LET-60 also functions non-autonomously to regulate neuroblast delamination (Parry and Sundaram, 2014), and, with LIN-12/Notch, controls development of the excretory tube (Abdus-Saboor et al,, 2011). LET-60 also triggers multiple ERK-dependent events in the germline (Eisenmann and Kim, 1997; Arur et al., 2009). For the cell fate patterning amongst groups of equipotent cells, LET-60 functions downstream of LET-23/EGFR; for fluid homeostasis LET-60 functions downstream of EGL-15/FGFR (see WormBook chapter Canonical RTK-Ras-ERK signaling and related alternative pathways).

The first Ras effector discovered was yeast adenylyl cyclase, and its Ras binding domain is a series of distinctive leucine-rich repeats (LRRs). Researchers initially thought that mammalian oncogenic Ras signaling would similarly be through adenylyl cyclase, but metazoan adenylyl cyclases do not have the LRR domain. Instead, the major Ras effectors in mammalian cells were found to be Raf kinase, and later PI3 Kinase, RalGEF, and others (Repasky et al., 2004). Nonetheless, LRR domains are still relevant for Ras signaling across phyla, and multiple Ras partners contain an LRR domain. A critical member of this group was discovered as a suppressor of activated Ras: SOC-2/SUR-8 (mammalian Shoc2) functions as a scaffold for Ras and Raf, and may be a major regulatory point for Ras signaling (Selfors et al., 1998; Sieburth et al., 1998). Additionally, missense mutation of Shoc2 causes defects in the RASopathy spectrum (Cordeddu et al., 2009). RASopathies are a series of heritable disorders in which derepression of the $\mathrm{Ras} \rightarrow \mathrm{Raf} \rightarrow \mathrm{MEK} \rightarrow \mathrm{ERK}$ cascade causes heritable birth defects, mental retardation, and increased risk of cancer (Ratner and Miller, 2015). 
Oncogenic activating mutations in Ras have mutations at positions 12, 13, and 61 as canonical activating mutations, with different alleles predominating in different tumor types (Pylayeva-Gupta et al., 2011). Mutations have also been developed to select for different effector usage. In the background of the mammalian Ras G12V activating mutation, the E37G mutation confers selectivity for RalGEF: the E37G compromises Ras binding to Raf and PI3K effectors, but leaves Ras $\rightarrow$ RalGEF interactions intact (White et al., 1995; Rodriguez-Viciana et al., 2004). The G12V,E37G mutant Ras has been well validated in subsequent cancer studies (Hamad et al., 2002), thus providing an excellent tool for dissecting Ras $\rightarrow$ RalGEF signaling (see Section 2.7). The let-60(s1124) (E37K) mutation was isolated as a strong loss of function allele, and mutates the same E37 residue as the RalGEF-selective allele, presumably blocking Raf binding. In mammals Ras T35S and Y40C are selective for Raf and PI3K activation, respectively (White et al., 1995); of these, T35S and E37G have been validated in C. elegans (Zand et al., 2011).

A series of loss-of-function, dominant-negative, and gain-of-function alleles defined the let-60 locus (also known as lin-34, from the gain-of-function alleles) (Beitel et al., 1990; Han and Sternberg, 1991). Isolated based on vulval development phenotypes (or lethality from strong alleles due to failed induction of the excretory duct cell, see below), this collection of let-60 alleles reflects the diversity of Ras alleles defined in other organisms.

The reference gain-of-function let-60 allele is $n 1046 g f$, which causes a G13E substitution. Oncogenic position 13 alleles in Ras are found in cancers and are moderately activating. Four additional G13E activating alleles have been found for let-60 ( n1700, n1849, sy103, sy130), and all cause similar phenotypes. Two non-canonical gain-of-function alleles (i.e., not position 12, 13, or 61 mutations found in cancer) have also been isolated in screens: ay75gf,ts (G60R) confers a semi-dominant and temperature-sensitive defect in fluid homeostasis, consistent with constitutive activation of the EGL-15/FGFR cascade that regulates that process; and ay75gf,ts confers less penetrant fertility and vulval hyper-induction than the canonical G13E alleles like n1046 (Schutzman et al., 2001). let-60(ga89gf,ts) (L19F) confers semi-dominant and temperature-sensitive defects in fertility, consistent with activation of the Raf-MEK-ERK cascade at multiple points in the germline, but only weakly induces vulval hyper-induction (Eisenmann and Kim, 1997; Arur et al., 2009). Notably, let-60(n1046gf) alone promotes neither fluid homeostasis defects nor sterility, though it can do so in sensitized backgrounds. Though all three of these defects are Raf-dependent, the Raf cascade is evidently activated at different levels in different tissues, or different sets of co-effectors are activated in different tissues. Also, the absence of identified completely activating lesions at positions 12 and 61 suggests that such mutants would be lethal or sterile.

Dominant-negative let-60 alleles were also identified in mutant screens (Han and Sternberg, 1991). let-60 alleles sy101 and sy99 (G10R), sy94 (K16N), and sy93 (D119N) all confer a dominant-negative vulvaless phenotype. None of them corresponded to canonical S17N dominant-negative mutations identified from mammals, perhaps, as hypothesized for strong gain-of-function mutations, due to potential toxicity of strong alleles.

\subsection{RAS-1}

ras-1 encodes the ortholog of mammalian R-Ras1 and R-Ras2/TC21. Very little has been done with the $C$. elegans RAS-1, with one exception. RASopathies are a series of mostly overlapping syndromes caused by mutations that weakly activate the Ras $\rightarrow$ Raf $\rightarrow$ MEK $\rightarrow$ ERK MAP kinase cascade (Rauen, 2013). In a RASopathy patient, putative activating mutations in R-Ras were found. Heat-shock induction of $C$. elegans RAS-1 harboring the homologous mutation induced weak vulval morphogenesis defects compared to wild-type RAS-1, suggesting that RAS-1 may contribute to LET-60/Ras-LIN-45/Raf signaling (Flex et al., 2014), with the caveat that ectopic over-expression of mutationally activated RAS-1 could inappropriately engage effectors that typically associate with LET-60. R-Ras, TC21, and M-Ras (RAS-2, Section 2.3) share the trait of binding the RA domain of RalGEFs in vitro (Rodriguez-Viciana et al., 2004), raising the possibility that RAS-1 or RAS-2 could activate RalGEF (see Section 2.7). However, deletion alleles of neither ras-1 nor ras-2 conferred vulval phenotypes consistent with RalGEF interaction, but the double mutant has not been described (D.Reiner, unpublished data).

A key observation in mammalian R-Ras biology was the finding that the semaphorin receptors, plexins, function as ligand-dependent GAPs with specificity for R-Ras and M-Ras. R-Ras (and M-Ras) in turn activate integrins, so semaphorin-plexin signaling inhibits integrin activation, thus allowing growth cone collapse (Puschel, 2007). Later studies challenged this model, instead arguing that plexins function as Rap-specific GAPs (Wang et al., 2012; Wang et al., 2013). Plexins also impact Rho family signaling, so the story is liable to be complex. In $C$. elegans, plexins function in collaboration with Eph receptors to coordinate development of sensory rays in the male tale and embryonic ventral enclosure (Ikegami et al., 2004; Ikegami et al., 2012), but connections to Ras family members have not been observed. 


\subsection{RAS-2}

ras-2 encodes the ortholog of mammalian M-Ras/R-Ras3. RAS-2 is one of the C. elegans Ras-like proteins whose effector-binding domain is not absolutely conserved: a mammalian M-Ras effector-binding domain Ser that is conserved in human and $C$. elegans LET-60/Ras, Rap1, and RAS-1 is a Gln in RAS-2, perhaps indicating that RAS-2 effector partners are not conserved with mammalian partners. A major point of interest with mammalian M-Ras is that it binds the LRR domain of Shoc2, thus regulating the Ras-Raf scaffold and regulating a major oncogenic cascade (Rodriguez-Viciana et al., 2006; Young et al., 2013). However, no functions have been associated in RAS-2 in C. elegans.

\subsection{RAP-1}

rap-1 encodes the ortholog of mammalian Rap1A/Krev-1 and Rap1B. Rap1 started out with misdirection and continues to confound simple categorization. Rap1/RAP-1, as well as M-Ras and R-Ras/TC21/RAS1, have effector binding domains identical to those of LET-60/Ras. This observation, coupled with similar overall structure across the rest of the proteins, led early researchers to believe that extensive functional redundancy existed across the protein group. Subsequent experiments argue otherwise, with only Ras itself demonstrating widespread and central roles in biology across species. The effector domain identity between Ras and Rap1, then, remains enigmatic. Perhaps the functional differences are defined by divergent C-terminal membrane localization sequences, resulting in interaction with different partners in different subcellular compartments (Prior and Hancock, 2012).

Rap1 was initially identified as a Ras-like protein (Ras proximal), and over-expression assays led cancer researchers to believe that Rap1 functioned as a competitive inhibitor of Ras activation of Raf kinase (Frische and Zwartkruis, 2010). More precise experiments later disproved this notion, but the possible connection between Rap1 and Raf is still controversial in the mammalian literature, compounded by barriers to genetic analysis presented by two Rap1 genes, three Ras genes, and three Raf genes. In contrast, experiments with the single Rap1 gene in Drosophila support the model that Rap1 binds and activates Raf1 (Mishra et al., 2005; Mavromatakis and Tomlinson, 2012). The issue remains unaddressed in C. elegans.

In most systems Rap1 (and Rap2) are known for regulating biology of cell junctions, and many Rap1 (and Rap2) effectors impact junctional biology and/or morphogenesis ( Kooistra et al., 2007). In C. elegans, RAP-1 and RAP-2 redundantly function in the hypodermis to control larval molting, such that few double mutant animals survive. RAP-1 and RAP-2 are jointly activated in this process by PXF-1 ortholog of the mammalian RapGEF PDZGEF (Pellis-van Berkel et al., 2005). The PXF-1 larval arrest mutant phenotype was partially rescued by heat-shock expression of the RAP-1 G12V gain-of-function. PXF-1 promoted GTP exchange specifically on RAP-1 and RAP-2, but not LET-60/Ras or RAL-1. The pxf-1 promoter::GFP fusion was expressed in hypodermis in pulses corresponding with larval molts, consistent with function at that time and place. Other structural defects were characterized. Perhaps surprisingly, compared to the role of Rap1 in other systems, rap-1 mutant animals are viable. The rap-1(pk2082) nonsense allele was initially characterized as conferring growth and structural defects, but additional outcrossing and comparison to the tm861 deletion allele (Frische et al., 2007; D. Reiner, unpublished results) indicates that the growth defects of rap- 1 mutant animals, if any, are mild.

The expected RAP-1 contribution to junctional biology was found in a synthetic lethal RNAi screen, which identified the related Ras family small GTPase, RAL-1, as providing redundant functions in hypodermal junction formation and cadherin complex localization, resulting in rupturing of embryos (Frische et al., 2007) (see Section 2.7). Components of the RAL-1 signal were analyzed, but RAP-1 was not. RAP-2 apparently played no role in this redundant event. The endogenous rap-1 locus was tagged, using CRISPR technology, with fluorophore mNeonGreen at the 5' end, creating an N-terminally tagged protein that localized to most or all plasma membranes (Dickinson et al., 2015).

RNAi screens for defective male somatic gonad identified both RapGEF PDZGEF/PXF-1 and RAP-1 (Kalis et al., 2010), and pxf-1 was identified in an RNAi screen for resistance to paralysis induced by the acetylcholine esterase inhibitor aldicarb (Sieburth et al., 2005). The rgef-1 gene encodes the C. elegans ortholog of mammalian CalDAG GEF/RasGRP2, which promotes exchange on both Ras and Rap1 (Raaijmakers and Bos, 2009). The RGEF-1 exchange activity is conserved in $C$. elegans but the reported RapGEF function in olfaction functions through LET-60/Ras and not RAP-1 (Chen et al., 2011). 
All RapGEFs are conserved in $C$. elegans, but many RapGEFs also activate Rap2, and some can activate Ras and other Ras family members. In addition to the aforementioned PXF-1/PDZGEF and RGEF-1/CalDAGGEF, $C$. elegans expresses the putative RapGEF EPAC-1/cAMP-activated RapGEF, but only phenotypes associated with DRN-1 (ortholog of mammalian Di-Ras1/Rig, Di-Ras2, and Di-Ras3/Noey2/ARHI, see Section 2.9) have been identified (Tada et al., 2012). The RapGEF C3G/Y34B4A.4 is encoded in C. elegans, but no phenotype is associated with this protein. Worms also encode the combination RapGEF and phospholipase epsilon PLC-1/PLC- $\varepsilon$. PLC- $\varepsilon$ has been identified as an effector for Ras in C. elegans and other systems (Bunney and Katan, 2006). PLC-1 is required for fertility and ovulation (Shibatohge et al., 1998; Kariya et al., 2004) and embryonic morphogenesis (Vazquez-Manrique et al., 2008). It is unclear whether these activities are connected with small GTPase signaling. Oddly for Ras family GEFs, across metazoans PDZGEF, EPAC, and PLC- $\varepsilon$ also contain Ras Association (RA) domains, which potentially serve as Rap1 effectors or points of cross talk with other related Ras family proteins that can also bind RA domains (Raaijmakers and Bos, 2009). Large sets of Rap effectors have been characterized, and many of them are shared with Rap2.

Rap1 and Rap2 have also been suggested to be inhibitory substrates of the semaphorin receptor, plexin. Activated plexins functions as GAPs, though whether for Raps, R-Ras, or both is unclear (Puschel, 2007; Wang et al., 2012; Wang et al., 2013). The Rap subfamily is also notable for having a Thr at position 61 (Ras numbering, compared to the Gln at position 61 in most Ras family members). This change is thought to confer GAP specificity (Daumke et al., 2004), and is shared only with the Di-Ras subfamily among Ras family members.

\subsection{RAP-2}

rap-2 encodes the ortholog of mammalian isozymes Rap2A, Rap2B, and Rap2C. Rap2 and Rap1 junctional functions in other systems are significantly overlapping, though individual activities have been defined (Raaijmakers and Bos, 2009). In C. elegans the only demonstrated biological outcome of RAP-2 activity is its redundant regulation of molting in concert with RAP-1, downstream of the GEF PXF-1 (see Section 2.4) (Pellis-van Berkel et al., 2005). Mammalian Rap2 signals to Ezrin via TNIK, and this general relationship was supported in $C$. elegans by localized enrichment of phospho-Ezrin in a rap-2 null mutation background (Gloerich et al., 2012). Beyond this loose connection Rap2 effectors have not been characterized.

The rap-2(gk11) mutation has been shown, through redundancy with the rap-1 null allele, to strongly reduce rap-2 function, but single mutant animals are superficially wild type (Pellis-van Berkel et al., 2005). No other functions have been associated with RAP-2.

\subsection{RAP-3}

rap-3 encodes a divergent Rap-like protein. Overall GTPase domain structure, hypervariable region, and CAAX sequences are most similar to Rap proteins, particularly Rap1. However, key changes in the RAP-3 core effector-binding region, particularly a substitution of Ala for a Pro conserved in all Ras family members, suggests that RAP-3 does not functionally overlap its phylogentically conserved brethren RAP-1 and RAP-2. RAP-3 is conserved in related nematodes but not humans or Drosophila, suggesting that RAP-3 represents a nematode-specific addition that may engage distinct effectors. No functions have been ascribed to RAP-3.

\subsection{RAL-1}

ral-1 encodes the ortholog of mammalian RalA and RalB. By RNAseq the C. elegans ral-1A transcript encodes a Ral ortholog that is well conserved across the entire GTPase. The minority ral- $1 B$ transcript is predicted to encode a RAL-1B protein with a unique 41 residue N-terminal extension that is unprecedented in other species. It is unclear whether ral-1B transcript produces protein, and it has not been detected in related nematode species.

The function of RAL is to promote $2^{\circ}$ vulval cell fate (see Canonical RTK-Ras-ERK signaling and related alternative pathways) and shorten lifespan (WormBook chapters Dauer and Obesity and the regulation of fat metabolism). The main Ral claim to fame in mammals is as a non-canonical effector of the Ras GTPase (see Section 2.1). This is a case of one Ras subfamily activating another Ras subfamily: activated Ras binds the RA (Ras Association) domain of RalGEF, thereby recruiting the GEF to the membrane and stimulating GTP exchange activity of Ral. Ral is generally in the vicinity of its activated GEF since, like Ras, Ral is membrane localized via its C-terminus. Indirect genetic results from Drosophila suggest that Rap1 (Section 2.4) also binds and activates RalGEF (Mirey et al., 2003), but these results are also consistent with parallel signaling. In all systems examined, 
Rap1, like Ras, can bind the RalGEF RA domain in vitro or in yeast two hybrid assays, but evidence is lacking in vivo, including in C. elegans vulval patterning (Zand et al., 2011) (see below).

Unusual for a GEF and GTPase pair, RalGEF and Ral are monogamous: RalGEFs are thought to activate only Ral, and Ral is activated only by RalGEFs (Feig, 2003). Invertebrates have single RalGEF genes (encoded by rgl-1 in C. elegans) compared to four in mammals. Deletion mutants of RalGEF are superficially wild type (T. Zand, K. Monahan and D. Reiner, unpublished results). Mammals and Drosophila, but not C. elegans, also encode Ras-independent RalGEFs, RalGPS1/2, that do not have an RA domain and are thought to be regulated by lipid signaling, but little is known about these proteins.

Despite exceptionally high sequence identity throughout the Ral GTPase domain, mammalian RalA and RalB, though both contributing to cancer, perform distinct functions in the cell. The RalA mouse knockout caused embryonic lethality while the RalB knockout is viable. The RalA/B double knockout is more severe than the RalA single knockout, consistent with diverging but also overlapping functions (Peschard et al., 2012). This difference between RalA and RalB functions is conferred by the hyper-variable C-terminal membrane targeting sequences (Feig, 2003; Camonis and White, 2005). C. elegans and Drosophila encode single Ral proteins.

A regulatory switch from Ras $\rightarrow$ Raf to Ras $\rightarrow$ RalGEF $\rightarrow$ Ral resolved a contradiction between morphogen gradient and sequential induction models of vulval cell fate patterning. Understanding this switch requires understanding of vulval cell fate patterning (see Vulval Development; Sherwood et al., 2005; and Canonical RTK-Ras-ERK signaling and related alternative pathways). A long-standing controversy in the field is the contradiction between two general models of vulval patterning by EGF. Classic developmental experiments and manipulation of strength of EGF and EGFR activity led to the morphogen gradient model (Figure 7). This model posits that an EGF gradient dictates the $3^{\circ}-3^{\circ}-2^{\circ}-1^{\circ}-2^{\circ}-3^{\circ}$ pattern of vulval fates, with $1^{\circ}$ fate being determined by closest proximity to the AC, and hence EGF (Sternberg and Horvitz, 1986; Sternberg, 1988; Sternberg and Horvitz,1989; Katz et al., 1995; Katz et al., 1996). Mutant screens identified under-induced (Vulvaless; Vul) and hyper-induced (Multivulva, Muv) mutants defining the core genes involved in patterning the vulva (Ferguson et al., 1987). Cloning of these genes led to identification of two signaling axes, EGFR $\rightarrow \rightarrow$ Ras $\rightarrow$ Raf $\rightarrow$ MEK $\rightarrow$ ERK MAP kinase, and Notch $\rightarrow$ CSL transcription factor, that are necessary for $1^{\circ}$ and $2^{\circ}$ signals, respectively (reviewed in WormBook chapters: LIN-12/Notch signaling in C. elegans; Notch signaling: genetics and structure; Vulval development; Canonical RTK-Ras-ERK signaling and related alternative pathways). The sequential induction model arose from findings that EGFR and Notch are necessary and sufficient in $1^{\circ}$ and $2^{\circ}$ cell fates, respectively (Seydoux and Greenwald, 1989; Koga and Ohshima, 1995; Simske and Kim, 1995). Initial EGF signal activates the $\mathrm{Ras} \rightarrow \mathrm{Raf} \rightarrow \mathrm{MEK} \rightarrow$ ERK cascade to induce $1^{\circ}$ cell fate. In presumptive $1^{\circ}$ cells, this ERK MAP kinase cascade also induces Notch ligands, which induce two neighboring VPCs to become $2^{\circ}$ (Chen and Greenwald, 2004). Importantly, $1^{\circ}$ and $2^{\circ}$ cell patterning signals are antagonistic. Notch activates expression of ERK phosphatase (LIP) in presumptive $2^{\circ}$ cells to block inappropriate ERK activity (Berset et al., 2001), while LIN-12/Notch receptor is internalized and degraded in presumptive $1^{\circ}$ cells to similarly block inappropriate Notch activation in presumptive $1^{\circ}$ cells (Shaye and Greenwald, 2002; Shaye and Greenwald, 2005). Importantly, transcription of many modifier genes is re-programmed between initial EGF induction and the first cell division of VPCs (Berset et al., 2001; Yoo et al., 2004; Berset et al., 2005; Yoo and Greenwald, 2005; Zand et al., 2011). Consequently, vulval development can be divided into two stages: initial specification, which is reversible, and commitment/reinforcement, which is irreversible and results in final cell fate (Vulval development). 


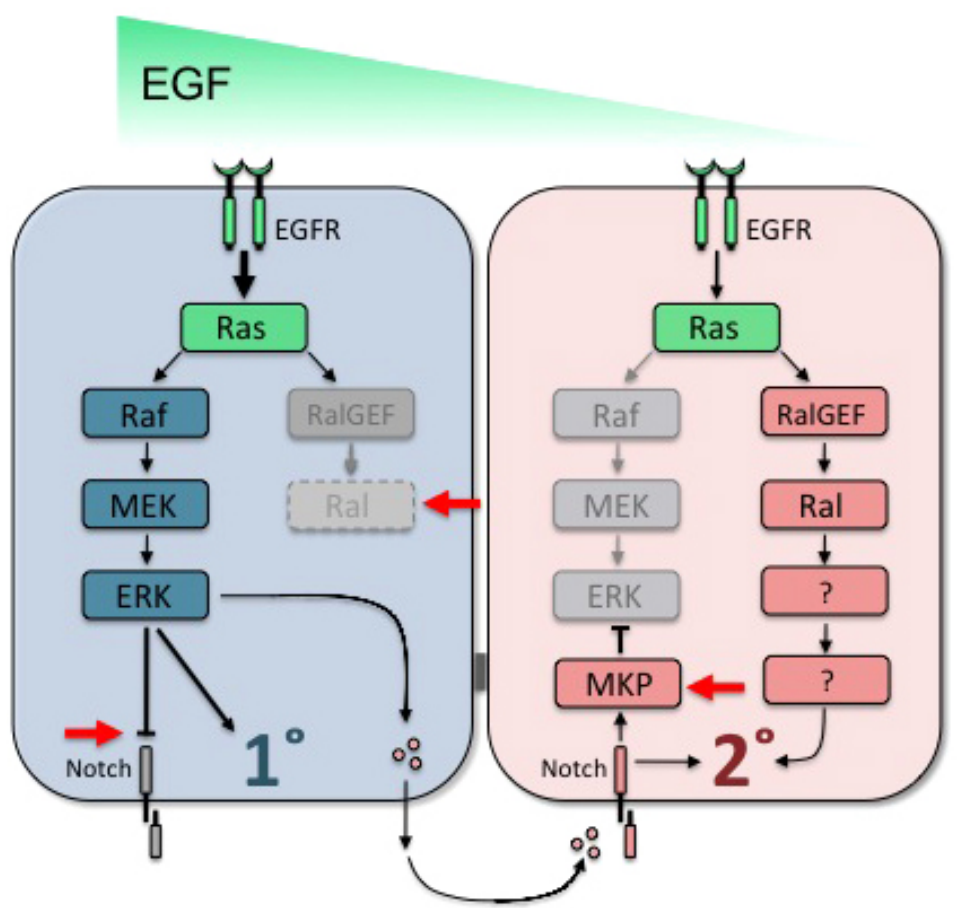

Figure 7. Vulval Ras switches effectors depending on cell position in the EGF gradient. Two models of vulval cell fate patterning, the morphogen gradient model and the sequential induction model, were formerly considered contradictory (Kenyon, 1995). The discovery that Ras $\rightarrow$ RalGEF $\rightarrow$ Ral mediates lower dose EGF signal to promote $2^{\circ}$ fate reconciled the two models, and the combined model is shown here. Red arrows show key points at which signals are switched from supporting one cell type to the other. LIN-12/Notch is internalized and degraded in presumptive $1^{\circ}$ cells to prevent contradictory Notch signaling. Notch-dependent transcriptional up-regulation of LIP-1 ERK phosphatase in presumptive $2^{\circ}$ cells represses inappropriate MPK-1/ERK activation. Ral promoter activity is excluded from presumptive $1^{\circ}$ but persists in presumptive $2^{\circ}$ cells, thus preventing inappropriate activation of Ral.

That conflicting signals exist in VPCs prior to their final fate commitment allowed identification of additional signaling cascades (Zand et al., 2011; Nakdimon et al., 2012), particularly identification of Ras $\rightarrow$ RalGEF $\rightarrow$ Ral, which explains how high and low doses of EGF can induce different outcomes in Ras signaling. In presumptive $2^{\circ}$ cells Ras also signals, but through RalGEF $\rightarrow$ Ral to promote $2^{\circ}$ fate rather than Raf $\rightarrow$ MEK $\rightarrow$ ERK (Figure 7). $\mathrm{Ras} \rightarrow \mathrm{RalGEF} \rightarrow \mathrm{Ral}$ signal is dispensable for core patterning of the vulval precursor cells (Zand et al., 2011), but is an important positive regulator of Notch signal in VPCs. Ras $\rightarrow$ RalGEF $\rightarrow$ Ral thus mediates lower dose EGF signal in the morphogen gradient. And so the gradient is overlaid on the $1^{\circ}-2^{\circ}$ sequential induction by Ras $\rightarrow$ Raf $\rightarrow$ MEK $\rightarrow$ ERK and Notch. This work resolved the controversy in the field of how to reconcile conflicting observations of sequential induction vs. morphogen gradient patterning of the VPCs (Katz et al., 1995; Kenyon, 1995; Koga and Ohshima, 1995; Simske and Kim, 1995; Katz et al., 1996). This Ras switch in effectors is orchestrated at a cellular level by two complementary mechanisms. First, a ral-1 transcriptional reporter is initially expressed in all of the vulval precursor cells, but after the onset of EGF signaling, expression is excluded from presumptive $1^{\circ}$ cells while persisting in presumptive $2^{\circ}$ cells. Thus unknown transcriptional circuitry insulates presumptive $1^{\circ}$ cells from inappropriate Ras $\rightarrow$ RalGEF $\rightarrow$ Ral signaling. In addition, the Ras $\rightarrow$ Raf output of ERK phosphorylation is quenched in presumptive $2^{\circ}$ cells by Notch-dependent transcription of the LIP-1/MKP/ERK phosphatase and other Notch-dependent transcriptional client genes (Berset et al., 2001; Yoo et al., 2004; Berset et al., 2005; Yoo and Greenwald, 2005).

This study was the first example of Ras dynamically switching effectors; it was previously assumed that different cell types were programmed to respond with different effectors. But Ras and its effectors Raf and RalGEF are all present in naïve VPCs before the onset of EGF signaling. Thus, a key unresolved question is whether and how Ras discriminates between partners that appear otherwise equivalent (Reiner, 2011). This mechanism may contribute to the $99.8 \%$ accuracy of VPC patterning (Braendle and Felix, 2008), particularly given the centrality of $\mathrm{Ras} \rightarrow \mathrm{Raf}$ in the process and the pitfalls of inappropriate signaling.

As with many ancient small GTPases, RAL-1 and Drosophila Ral share exceptionally high identity with RalA and RalB throughout the GTPase domain, including $100 \%$ identity in the core effector-binding region, suggesting that RAL-1 and RalA/B regulate a common suite of effectors. Because of their roles in RalA/B-dependent oncogenic 
signaling, three canonical Ral binding partners have received most of the attention. RalBP1 (also known as RLIP76, RLIP1, and RIP1) contains a RhoGAP domain shown in vitro to be selective for Rac and Cdc42, has membrane transporter ATPase activity, and can scaffold proteins participating in endocytosis (Gentry et al., 2014). Phosphorylation of the C-terminus of mammalian RalA relocalizes it to the mitochondrial membrane, where it signals through RalBP1 to promote mitochondrial fission at mitosis (Kashatus et al., 2011). C. elegans RLBP-1 contains Ral-binding and RhoGAP domains, but it is unknown whether all of the mammalian RalBP1 functions are conserved and what functions RLBP-1 performs. Biological functions of $C$. elegans RLBP-1 are unknown.

Sec5 and Exo84 are well-validated binding partners of mammalian RalA/B and are essential and non-essential components of the exocyst complex, respectively. The exocyst is a phylogenetically conserved heterooctomeric complex that controls exocytosis and diverse aspects of cell behavior (Shirakawa and Horiuchi, 2015; Wu and Guo, 2015). The yeast exocyst uses Rab and Rho for this purpose, but metazoans seem to have replaced those functions with Ral (Novick and Guo, 2002). ral-1(tm2760), a deletion in intron 3 that perturbs the splice donor sequence, confers sterility (Zand et al., 2011), as does the putative null deletion tm5205. Depletion of both maternal and zygotic ral-1 disrupted cell polarity, tubulogenesis, and exocytosis, and these functions are closely associated with the PAR proteins (Armenti et al., 2014). Disruption of maternal and zygotic SEC-5, a central exocyst complex component from yeast to humans, confers the same phenotype, suggesting that RAL-1 participation as a member of the exocyst regulates fundamental cell biological events. Null mutants for RGL-1/RalGEF are superficially wild type (T. Zand, K. Monahan, and D. Reiner, unpublished results), consistent with the RAL-1 functions in the exocyst being activation-independent.

Exo84 and Sec5 serve as RalA/B docking sites for the exocyst in mammals. Exo84 and Sec5 also function as signaling scaffolds, coupling Ral to downstream activities. Sec5 mediates RalB-dependent activation of TBK1 in innate immunity and tumor survival (Chien et al., 2006). Mammalian Exo84 mediates RalB-dependent activation of autophagosome assembly (Bodemann et al., 2011) and mTORC1 activation (Martin et al., 2014) (see below). But many signaling intermediaries that execute these Ral-Sec5- and Ral-Exo84-dependent functions remain unknown.

In C. elegans, Exo84 and Sec5 have only been connected with Ral in regulation of cadherin-based junctions in embryonic hypodermal cells (Frische et al., 2007). In a screen for hypodermal phen otypes, $\mathrm{rgl}-1$ and $\mathrm{ral}-1$ were recovered as genes that confer a synthetic lethal RNAi phenotype in animals mutant for the RAP-1 small GTPase (Section 2.4). Also identified were genes encoding Ral binding partners SEC-5 and EXOC-8. No other characterized Ral-dependent process has used both exocyst partners, so perhaps Ral engages two genetically separable but required activities in this process. $r l b p-1$ and genes encoding other exocyst components were not synthetic lethal with rap-1 mutations. RAL-1 and RAP-1 signaling redundantly control cadherin complex localization to junctions of embryonic hypodermal cells, and hence double mutants disrupt epithelial integrity, migration, and morphogenesis. Upstream input has not been tested, particularly by LET-60/Ras, which might be expected. RAP-2 (Section 2.5) was not found to play a role in this process.

The existence of mammalian RalGAP biochemical activity was known for decades (Emkey et al., 1991), but the molecular identity of RalGAP was only identified relatively recently (Shirakawa et al., 2009; Chen et al., 2011). RalGAP is a heterodimer consisting of a catalytic $\alpha$ subunit and regulatory $\beta$ subunit. The homology of the GAP domain and unusual regulatory architecture draws a parallel to the Tuberous Sclerosis Complex (TSC) heterodimeric RhebGAP (see Section 2.8). Determination that Akt phosphorylation inhibits RalGAP like it does TSC/RhebGAP further underscores the similarity between RalGAP and TSC/RhebGAP (Chen et al., 2011; Leto et al., 2013). However, nematode genomes do not express TSC orthologs. C. elegans hgap-1 and hgap-2 encode orthologs of RalGAP $\alpha$ and $\beta$ subunits, respectively. Based on the reasoning that the ancestral nematode must have had compensating RhebGAP activity to allow loss of TSC/RhebGAP, RalGAP is a candidate for a shared Ral and Rheb GAP in C. elegans, hence the intentionally noncommittal name of Heterodimeric GTPase Activating Protein (HGAP). Putative null mutation of hgap- 1 or hgap-2 reduced lifespan, as predicted for a TSC-like complex (Martin et al., 2014). This reduced lifespan phenotype was reversed by ral-1(RNAi), consistent with the HGAP complex repressing RAL-1 activity as a RalGAP but leaving potential function as a RhebGAP unresolved. HGAP-2 was previously identified in an RNAi screen for decrease of DAF-2/InsR mutant longevity (Samuelson et al., 2007), but because RalGAP was as yet undiscovered, its role in signaling was not recognized. RAL-1 was identified as an aging gene in an RNAi screen for sensitivity to oxidative stress (Kim and Sun, 2007).

Based on the expectation that a common ancestor of $C$. elegans and mammals used both RalGAP and TSC/RhebGAP to regulate TOR, crosstalk was found between mammalian RalGAP and TSC/RhebGAP, and RalB was found to signal through Exo84 to directly activate TORC1. However, RalB localized TORC1 to the plasma membrane to mediate RalB-dependent invasion activity in cell culture, contrasting with canonical Rheb-TORC1 
activation of anabolic metabolism at the lysosome. Thus Ral and Rheb may recruit TORC1 to different subcellular compartments to mediate distinct activities (Martin et al., 2014). RalB $\rightarrow$ Exo84 activates autophagy under starvation conditions (Bodemann et al., 2011). Autophagy is catabolic, is generally regulated in opposition to TORC1, and is directly inhibited by TORC1 kinase activity. Thus these two activities of RalB in cell culture may be contradictory. Alternatively, perhaps RalB switches between promoting catabolism when cells are starved and anabolism when cells are well fed. These signaling permutations suggest a complex regulatory dance in the interpretation of upstream nutritional status by Ral and Rheb.

In support of a mammalian study showing RalA-JNK-FOXO4 signaling in stress response, exogenous mammalian GST-JNK was phosphorylated in response to heat shock in a RAL-1 dependent manner in worm extracts, and upon heat shock transgenic $C$. elegans FOXO/DAF-16::GFP translocated to the nucleus in a RAL-1 dependent manner. Exogenous tagged mammalian RLF/RalGEF and RalA bound to the JIP1 JNK scaffold upon stimulation by reactive oxygen species, suggesting that Ral contributes to a central scaffold to promote JNK activation (van den Berg et al., 2013).

\subsection{RHEB-1}

rheb-1 encodes the single $C$. elegans ortholog of mammalian Rheb1 and Rheb2/RhebL1. While RHEB-1 is clearly most similar to mammalian Rhebs, certain sequence features of the nematode protein are unconventional. First, mammalian and Drosophila Rhebs diverge from other Ras family members at Ras oncogenic mutation positions 12 and 13 (Ras numbering), having an "RC or "RS" instead of "GG". C. elegans RHEB-1 has a "PH" at positions 12, 13 (Figure 8). Second, though the mammalian and Drosophila Rheb core effector-binding domains are distinct from those in the rest of the Ras family, certain sequence elements are conserved throughout. Strikingly, the C. elegans RHEB-1 core effector-binding domain diverges at multiple positions, including the third position Proline conserved in most Ras family members (Figure 8). Even yeast Rhebs, S. cerevisiae Rhb ${ }^{+}$and $S$. pombe Rheb, have better conserved effector-binding domains than $C$. elegans RHEB-1. RHEB-1 may therefore be the exception the proves the rule in co-conservation of small GTPases and effector domains (see above): Rheb across species only has a single described effector, the Target of Rapamycin (TOR) Ser/Thr kinase, so perhaps there is greater tolerance of changes in the Rheb core effector binding region, which are presumably reflected in its downstream partner. Alignment of the $C$. elegans, Drosophila, and two human Rheb sequences throughout the GTPase domain indicates that, in C. elegans, Rheb has converged toward Ral (D. Reiner, unpublished observations) (Figure 8). As a control, Drosophila Rheb has not converged toward Drosophila Ral. Perhaps this convergence accompanied the evolutionary loss of TSC in $C$. elegans: if the HGAP-1/2 RalGAP performs both RalGAP and RhebGAP activities in $C$. elegans, it stands to reason that the described changes in RHEB-1 to become more Ral-like facilitated co-regulation of these protein, which are separately regulated in other systems. 
RalA

RalB

DRal

RAL-1

Rheb 1

Rheb2

DRheb

RHEB-1

RalA

RalB

DRal

RAL-1

Rheb1

Rheb2

DRheb

RHEB-1

RalA

RalB

DRal

RAL-1

Rheb1

Rheb2

DRheb

RHEB-1
LALHKV IMVGSGGVGKSALTLQFMYDEFVEDYEPTKADSYRKKVVLD GEEVQIDILDTAG LALHKV IMVGSGGVGKSALTLQFMYDEFVED YEPTKADS YRKKVVLD GEEVQIDILDTAG PALHKVIMVGSGGVGKSALTLQFM DEFVED YEPTKADSYRKKVVLD GEEVQIDILDTAG Q VHKVIMVGTGGVGKSALTLQFMY DEFVEEYEPTKADS YRKKVVLD GEECSIDILDTAG VRYRKVVILGYRCVGKTSLAHQFVEG FSEGYDPTVENT SKIVTLGKDEFHLHLVDTAG SKSRKIAILGYRSVGKSSLTIQFVEGQFVD Y DPT IENTFTKL ITVN OEYHLQLVDTAG TKERH I AMMGYRSVGKSSLCIOFVEGOFVD S DPT I ENTFTK I ERVKSODY IVKL IDTAG SLNRKVAVMGY PHVGKSALVLRFTQNIF PERY STIE OHSK IAAF HRDYHLRVTDTAG

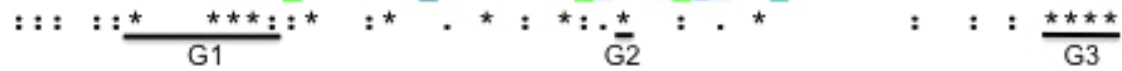
OEDYAA IRDNYFRSGEGFLCVF ITEMESFAATADFREQILRVKE-DENVPFLLVGNKSD QEDYAA I RDNYFRSGEGFLLVFS ITEHESFTATAEFREQILRVKAEEDK I PLLVVGNKSD OEDYAA I RDNYFRSGEGFLCVF S ITDDESFQATQEFREQILRVKN-DES I PFLLVGNKCD QEDYSA I RDNY YRSGEGF I CVF S ILDMESFEATNE FREQ ILRVKNSDSSVP IVLVGNKGD QDEYSILPYS FII GVHGYVLVY SVTSLHSFQVIESLYQKLHEGHG-KTRVPVVLVGNKAD QDEYS I FPQTYS ID INGY ILVY SVTS I KSFEVI KVI HGKLLDMVG-KVQI P IMLVGNKKD

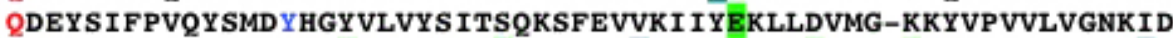
QQEYTVFPRSCSLD INGFI IVYA IDDRKSFEMCSN I YEK IVRTYG-DTS I P IVIVGNKTD

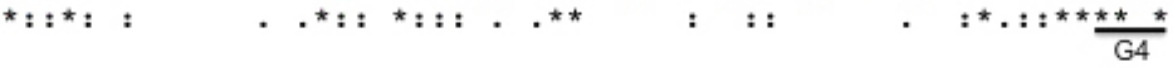

LEDKRQVSVEEAKNRAEQWNVNYVETSAKTRANVDKVFFDLMREIRAR

LEERRQVPVEEARSKAEEWGVQYVETSAKTRANVDKVFFDLMREIRTK

LNDKRKVPLSECQLRAQQWAVPYVETSAKTRENVDKVFFDLMREIRSR

MRD RVVSAELCRQRAEQWGCHYVETSAKRRENVDKVFY DLMREMKRR

LS PEREVQAVEGKKLAESWGATFMESSARENQLTQGIFTKVIQEIARV

LHMERV I SYEEGKALAESWNAAFLES SAKENQTAVDVFRRI I LEAEKM

LHQERTVSTEEGKKLAESWRAAFLETSAKONESVGDIFHOLLILIENE

LSTORVVRAEEGEELARQWDAKFVEITARES NRVHEVFELLLREIEIS

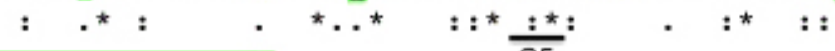

\section{Identical transvergent residues}

Convercent residues

Divergent residues

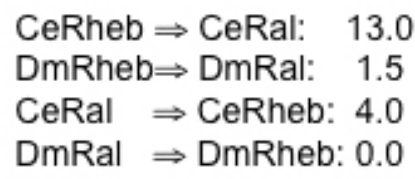

Figure 8. C. elegans Rheb has converged towards the Ral subfamily. The convergence was observed for $C$. elegans Rheb and Ral, but not Drosophila and human Rheb and Ral. Core GTPase sequences of human, fly, and worm Rheb subfamily and Ral subfamily proteins were aligned and scored for number of convergent vs. divergent residues. Residues that converge towards the consensus of the compared subfamily (here called "transvergent") and are identical (light green highlighting) were valued at 1 , with conservative transvergence (dark green highlighting) valued at 0.5 . True convergence of a residue from both subfamilies to a new residue type (light green letter) was also valued at 0.5 , while divergence (blue letter) was valued as zero. Events in the broad effector binding region (grayed, with core underlined) or $\mathrm{G}$ boxes were rated with higher impact (0.5 becoming 1.0$)$. By this standard $C$. elegans RHEB-1 converged toward C. elegans RAL-1, with Drosophila and human Rheb and Ral as negative controls (Fisher's Exact Test, $\mathrm{P}<0.0001$ ). $C$. elegans RHEB-1, and to a lesser degree $C$. elegans RAL-1, also contains significantly more divergent residues, perhaps reflecting a protein under more intense selective pressure than is typical. This convergent evolution may reflect the loss of TSC/RhebGAP but retention of HGAP/RalGAP in the $C$. elegans clade, so the RHEB-1 protein is adapted to co-regulation by RalGAP. Classic Rheb changes at positions 12 and 13 (Ras numbering) are indicated by purple (blue in RHEB-1).

Rheb is known as a key activator of TOR. TOR is associated with two complexes. TORC1 incorporates the co-factor Raptor and is a critical control point for promoting anabolic metabolism at the expense of catabolic anabolism (autophagy) (Dibble and Cantley, 2015). TORC2 mediates a feedback loop to Sgk and Akt in both mammals and C. elegans (Jones et al., 2009; Soukas et al., 2009). Rheb, and its inhibitory TSC RhebGAP, has different effects on TORC1 and TORC2: Rheb is well established as directly promoting TORC1 activation, but Rheb may inhibit TORC2 activation (Yang et al., 2006; Groenewoud and Zwartkruis, 2013).

Consistent with the model of RHEB-1 activating TOR and anabolic metabolism, which generally decreases lifespan, rheb-1(RNAi) extends lifespan (Honjoh et al., 2009). However, the same study found that in an intermittent fasting paradigm, which extends lifespan, rheb-1(RNAi) decreases lifespan. That is, RHEB-1 is required for the intermittent fasting lifespan extension. These contradictory observations suggest that RHEB-1- and TOR-dependent regulation of lifespan are likely to be context dependent. RHEB-1 signaling may regulate both insulin signaling and FoxA, which are anti- and pro-longevity, respectively (Honjoh and Nishida, 2011). RHEB-1 and TOR have also been implicated as repressors of the mitochondrial unfolded protein stress response (Haynes et al., 2007), which also impacts lifespan (Bennett and Kaeberlein, 2014). 
As noted in Section 2.7, C. elegans expresses all of the components of TOR system signaling except the tuberous sclerosis complex (TSC) RhebGAP (Long et al., 2002). TSC is present in Drosophila and all other metazoans besides nematodes; $S$. pombe has TSC while $S$. cerevisiae does not, but all of these organisms express Rheb, and Rheb loss is consistent with TOR loss biologically (van Dam et al., 2011). As noted in Section 2.7, the $C$. elegans HGAP complex could functionally replace the TSC/RhebGAP as well as function as a RalGAP. The decreased lifespan caused by disruption of HGAP-1 or HGAP-2 is consistent with the effect expected from derepressed RHEB-1.

The divergence of $C$. elegans RHEB-1 and absence of TSC/RhebGAP raises the question of whether Rheb function is conserved with other species. Deletion of $C$. elegans TORC1 components TOR and Raptor resulted in mid-larval arrest with an atrophied gut and RNAi depletion extended lifespan (Hara et al., 2002; Long et al., 2002; Vellai et al., 2003; Jia et al., 2004). Mutants for rheb-1 have not been described.

Mutating most Ras family members to a constitutively activate GTP-bound form is simple: mutate the G12, G13, or Q61 oncogenic Ras mutations (LET-60/Ras numbering). Not so with Rheb, because these positions are not conserved, and Rheb structure is atypical for the Ras family. In vitro mammalian Rheb1 is unusually highly GTP-bound, though GTP hydrolysis can be stimulated by addition of the TSC RhebGAP. Introducing the Q64L (Q61L in Ras numbering) mutation to Rheb1 confers a 2-3-fold increase in activity, much lower than activation conferred by homologous changes in other Ras family members (Inoki et al., 2003). The search for activating Rheb mutations in various systems has yielded only weak mutations (Mazhab-Jafari et al., 2014). With the high level of sequence divergence of $C$. elegans RHEB-1, it is uncertain whether the corresponding mutations would increase activity level. Ectopic expression of C. elegans RHEB-1(Q71L,Q72D) under control of its own promoter, an attempt to mutationally activate RHEB-1, confers weak TORC1 activation in an assay of rescue of fatty acid depletion (Zhu et al., 2013). Activation of RagA (see Section 7), another major input into TORC1, robustly rescued fatty acid depletion, suggesting that RHEB-1(Q71L,Q72D) is not constitutively active.

Dominant-negative mutations are useful for studying small GTPases function (see above) (Han and Sternberg, 1991). Canonical Ras dominant-negative mutation in mammalian Rheb1-S20N (S17N by Ras numbering) has no effect (Inoki et al., 2003), which, with the relatively high intrinsic Rheb1-GTP state could argue that Rheb1 does not require a GEF. Drosophila TCTP has properties expected from a RhebGEF (Hsu et al., 2007), with modest support from mammalian cells (Dong et al., 2009). In other contexts the mammalian TCTP does not perform RhebGEF activity or have genetic characteristics expected of RhebGEF in cell culture (Rehmann et al., 2008; Wang et al., 2008). Therefore it is still unknown whether there exists a RhebGEF in any system.

\section{9. $\mathrm{DRN}-1$}

drn-1 encodes the C. elegans ortholog of mammalian Di-Ras1/Rig, Di-Ras2, and Di-Ras3/Noey2/ARHI. The Di-Ras subfamily shares typical sequence features of the Ras family, with some differences that may confer Rap-like GAP specificity. The Rap family has a Thr at position 61 instead of the Gln of Ras (Ras numbering; see Section 2.4) and the Di-Ras family, including DRN-1, has a Ser at 61. Not surprisingly, mammalian Rap1GAP stimulates Di-Ras1 GTP hydrolysis, while typical RasGAPs do not (Gasper et al., 2010). The C. elegans Rap1GAP ortholog F53A10.2, and perhaps other Rap-specific GAPs may therefore also function as a GAP for DRN-1.

Di-Ras subfamily effector-binding domains contain modest but presumably important differences from the rest of the Ras family (Figure 5), suggesting that different signaling partners are used. The mammalian Di-Ras family members are all tumor suppressor genes (typically brain, breast, and ovarian), which is a departure from typical oncogenic role of Ras in cancer.

C. elegans DRN-1 is broadly expressed in neurons. The $o k 400$, $t m 2916$, and $t m 2926$ deletion alleles, all putative nulls due to dramatic disruption of conserved protein structure, confer resistance to the acetylcholine esterase inhibitor aldicarb, consistent with decreased cholinergic synaptic release in the mutants. This phenotype was transgenically rescued by putative gain-of-function, but not dominant-negative, drn- 1 constructs (Tada et al., 2012). Genetic interactions with $\mathrm{G} \alpha_{0}, G \alpha_{\mathrm{q}}$, and $\mathrm{G} \alpha_{\mathrm{s}}$ heterotrimeric G-protein $\alpha$ subunit mutants, all shown to regulate synaptic release, are consistent with DRN-1 functioning downstream of GSA- $1 / \mathrm{G} \alpha$ which stimulates cAMP production. Deletion of the cAMP-dependent RapGEF epac-1 also confers aldicarb resistânce (Tada et al., 2012), and thus by association may be a DRN-1 GEF. In the absence of known Di-Ras subfamily effectors, it is unclear whether the neurobiological phenotypes of $d r n-1$ mutants relate to the tumor suppressor phenotypes caused by loss of subfamily members in mammals. 
A singular feature of $d r n-1$ is the organization of the $d r n-1$ transcripts. The $d r n-1$ gene as annotated in Wormbase (WS247 and earlier versions) predicts two 5'UTR variants with different transcription initiation sites, one spliced and one not. However, using the first AUG start codon in hypothetical translation results in a 125 residue protein truncating the N-terminus of the GTPase domain and disrupting putative GTPase structure and effector binding. Mammalian Di-Ras subfamily members, like Ras itself, have an approximately 170 residue core GTPase domain, plus N- and C-termini extensions. There is no precedent for Ras family members with a truncated N-terminus; not only does that abolish guanine nucleotide cycling, it disrupts the protein structure used to interact with other proteins. Despite the absence of a conventional protein product, $d r n-1$ mutations confer a neuronal phenotype. Thus, $d r n-1$ is unlikely to be a pseudogene.

Notably, sequences $5^{\prime}$ of the first putative AUG, if translated, would produce a hypothetic N-terminus of DRN-1 homologous to mammalian Di-Ras subfamily members: the entire GTPase domain would be conserved (Figure 9). Transcript C54A12.4.2, based on RNAseq and RT-PCR data (V. Muñiz-Medina and D. Reiner, unpublished results), contains a long putative 5'UTR. There are no AUG or termination codons in frame upstream of the annotated AUG. We hypothesize that non-canonical translation initiation codons are used to produce a protein with the entire conserved GTPase domain: the minimal size based on sequence conservation and Ras family N-termini is a 192 residue DRN-1 protein. This hypothetical protein, the "minimal DRN-1 protein," has a functional GTPase domain, conservation of the entire GTPase sequence, and a function C-terminal membrane-targeting region. Therefore, we speculate that non-AUG translation initiation is used to express DRN-1.

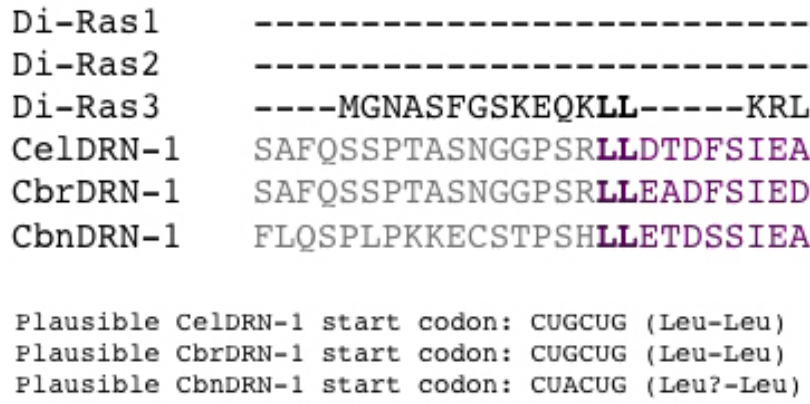

Figure 9. Alignment of human and Caenorhabditid Di-Ras subfamily predicted N-termini. GTPase domain sequences are in blue, established mammalian sequences in black, the inferred DRN-1 N-terminal extension in purple, and ambiguous further N-terminal extension in gray. Dileucine repeats are bolded. Di-Ras3 (ARHI, Noey2) has an N-terminal extension containing three dileucine motifs. Assuming a non-AUG initiation codon, transcript C54A12.4.2, predicted by RNAseq, aligns with upstream genomic sequence in C. briggsae (Cbr) and C. brenneri (Cbn), all of which could start with a dileucine repeat. Hypothetical translation of the overlapping transcript C54A12.4.1 also contains a dileucine, but it precedes a stop codon and so it may be a computational artifact. Detailed analysis of the $d r n-1$ gene structure may resolve these questions.

Though rare, there is a precedent for non-AUG initiation across many organisms (Ivanov et al., 2011). Where known, non-AUG codons generally follow the template of "NUG," with "CUG" being a common initiator. Leaky translation initiation mutants in $C$. elegans use start codons with similarity to "AUG," with "CUG" being among the most efficient (Maduzia et al., 2010; Zhang and Maduzia, 2010). A "CUGCUG" sequence lies in the C54A12.4.2 transcript in frame with the annotated AUG and with no intervening terminators, resulting in a hypothetical DRN-1 containing an N-terminal extension starting with LL dual leucine (Figure 9). The mammalian Di-Ras3/Noey2/ARHI protein has an N-terminal extension with three LL pairs. Perhaps there is a functional parallel between Di-Ras3/Noey2/ARHI and DRN-1 putative N-terminal extensions. The DRN-1 ortholog in other Caenorhabditis species suggests a long N-terminal extension with highly identical core Di-Ras sequences. The Wormbase entry for both $C$. briggsae and $C$. brenneri DRN-1, chosen because of available RNAseq data, predict the same gene organization: the first AUG sequence would result in a truncated protein, but alternate initiation codons would produce proteins conserved throughout their length with CeDRN-1.

Transcript C54A12.4.1, which partially overlaps C54A12.4.2, is not supported by RNAseq or RT-PCR data, so may be a computational artifact. C54A12.4.1 contains a long 5'UTR without the CUGCUG repeat. The 5'UTR does contain the sequences necessary to generate the hypothetical "minimal DRN-1 protein" that so closely aligns with the mammalian Di-Ras subfamily members.

\subsection{SSR-2}

ssr-2 encodes the C. elegans ortholog of mammalian Dexamethasone-induced Ras protein 1/RasD1/Dexras1 and Dexamethasone-induced Ras protein 2/RasD2/Dexras2/RHES. RasD1/2 are widely expressed, and expression is 
regulated by diverse hormones and stress response (Thapliyal et al., 2014). No functional data for SSR-2 have been reported. Because it is more distantly related, we did not include SSR-2 in alignments.

\section{The Rho family}

Rho-family GTPases in C. elegans and other systems have roles in cytoskeletal organization, cell polarity, cell migration, and cell membrane protrusion. Classic studies from the laboratory of Alan Hall, using mammalian cell culture, established that activated Rho promotes formation of stress fibers and focal adhesions (Ridley and Hall, 1992), activated Rac promotes lamellipodia and membrane ruffles (Ridley et al., 1992), and activated Cdc42 promotes spike-like filopodia (Nobes and Hall, 1995). The discovery of enormously diverse GEFs for the Rho family ( Rossman et al., 2005), plus many subsequent analyses of their functions, identified the importance of tight spatial regulation of Rho, Rac, and Cdc42 function by combinatorial activity of their GEFs for proper control of cytoskeletal activities and consequent morphogenetic actions (Goicoechea et al., 2014; Lawson and Burridge, 2014). Like the GEFs for the Rho family, GAPs for the Rho family are also quite diverse, but are under-studied.

Structurally, Rho family members are distinguished by the "Rho family insert" between $\beta$-strand 5 and $\alpha$-helix 4 (Valencia et al., 1991). The $C$. elegans genome encodes seven members of the Rho family of GTPases, and effector-binding regions are typically highly conserved, with some exceptions (Figure 10). Canonical members of the Rho-family GTPases include Rho ( rho-1), Rac ( ced-10), and Cdc42 ( $c d c-42$ ). The rac-2 gene is nearly identical to ced-10, but it is unclear if rac-2 is a functional gene or a non-functional duplication of the ced-10 locus, although RNAi of rac-2 enhances ced-10 (Struckhoff and Lundquist, 2003). mig-2 encodes an Mtl (MIG-2-like) GTPase, a family found in invertebrates with similarity to both Rac and Cdc42. Functionally, MIG-2 is similar to mammalian RhoG (deBakker et al., 2004). Two additional atypical Rho-like GTPases are CHW-1, which is similar to the Chp/Wrch family of GTPases in vertebrates (Kidd et al., 2015), and CRP-1, a Cdc42-like protein with a divergent effector-binding region (Jenna et al., 2005) (Figure 10).

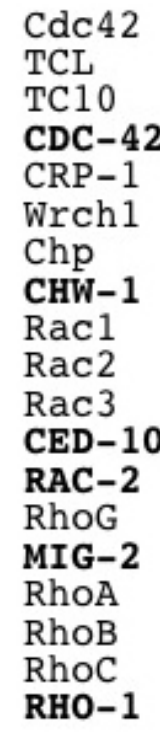

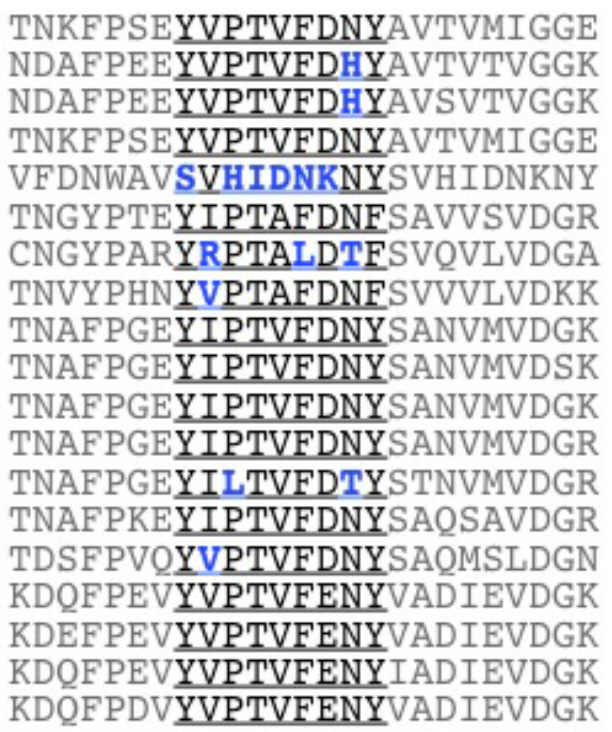

Figure 10. Effector-binding region of human vs. C. elegans Rho family members. The entire effector-binding region of human and $C$. elegans proteins was aligned. The core sequence, thought to govern direct protein-protein interactions, is underlined. Core residues not conserved in the subfamily are bolded and blue. Human orthologs are listed above C. elegans orthologs. C. elegans protein names have a dash and number. Some human proteins have alternate names (see Table 1).

Rho GTPases are key regulators of actin cytoskeleton dynamics, cell shape, and cell polarity. Based on four parameters of biochemical interaction with GTP-loading, GTP??S competition, hydrolysis, and exchange-the Rho GTPases were clustered according to functional similarity (Caruso et al., 2005). When all four traits were considered together, RHO-1, CDC-42, and CED-10 were in one functional cluster, with CDC-42 and CED-10 being closer together, and MIG-2 and CRP-1 were in another, suggesting that CRP-1 and MIG-2 have similar biochemical properties. By similarity of sequence, MIG-2 was closer to CED-10 and CDC-42, followed by RHO-1 and CRP-1. CHW-1 was not included in this analysis. Specific roles of the different Rho GTPases are described below. 


\section{1. $\mathrm{RHO}-1$}

Loss of rho-1 leads to embryonic lethality, and rho- 1 function has been ascertained using RNAi and by transgenic expression of constitutively active and dominant negative forms of rho-1. Conditional perturbation of rho- 1 function in adults results in dysfunction in numerous neuronal and non-neuronal functions and leads to death (McMullan and Nurrish, 2011), highlighting the central role of the RHO-1 GTPase in many biological processes throughout the life of the organism.

\subsubsection{Embryonic polarity and cytokinesis}

RNAi-mediated knockdown of rho- 1 activity resulted in early embryonic arrest, often with a failure of cytokinesis (Jantsch-Plunger et al., 2000; Spencer et al., 2001; Bringmann and Hyman, 2005; Morita et al., 2005; Motegi and Sugimoto, 2006). Embryos that arrested later in development displayed severe defects in tissue morphogenesis. These phenotypes could be explained by defects in Rho-mediated actin cytoskeleton organization (e.g., actin forms the contractile ring during cytokinesis and is involved in cellular migration and morphogenesis) (Schonegg and Hyman, 2006). Other mutations that affect embryonic morphogenesis and cytokinesis identified other genes that act with rho- 1 in this process. let-502 encodes a Rho kinase (mammalian ROCK or ROK), mel-11 encodes a myosin phosphatase (Wissmann et al., 1997; Wissmann et al., 1999; Piekny and Mains, 2002), and RHO-1 acts with the actin and myosin binding protein Anillin (ANI-1) in cytokinesis (Fotopoulos et al., 2013). These molecules likely affect actin-myosin cytoskeletal dynamics of the contractile ring during cytokinesis in response to Rho signaling (Spiga et al., 2013). Furthermore, RHO-1 is involved in establishing and maintaining the position of the PAR complex proteins in early embryonic polarity (Jenkins et al., 2006; Schonegg and Hyman, 2006; Anderson et al., 2008; Canman et al., 2008; Nakayama et al., 2009).

GAPs and GEFs also regulate RHO-1 in polarity and cytokinesis. The $c y k-4$ gene, identified in a screen for mutations affecting embryonic cytokinesis, encodes a Rho GAP that likely modulates RHO-1 activity in establishment of embryonic polarity (Jantsch-Plunger et al., 2000; Jenkins et al., 2006; Canman et al., 2008). The Rho GAPs RGA-3, RGA-4, and PAC-1, and the RHGF-2 GEF act with RHO-1 in cytokinesis (Schmutz et al., 2007; Schonegg et al., 2007; Anderson et al., 2008; Chan et al., 2015). The ECT-2 GEF (Drosophila Pebble) acts with RHO-1 in embryonic polarity and cytokinesis (Morita et al., 2005; Motegi and Sugimoto, 2006).

\subsubsection{Embryonic elongation}

LET-502 and MEL-11 also control embryonic elongation. After epiboly and ventral enclosure, hypodermal cells that surround the embryo contain circumferential rings of actin that contract, resulting in a "squeezing" of the embryo and a subsequent increase in length. LET-502 and MEL-11 have opposing roles in this process: LET-502 normally enhances contraction and MEL-11 normally inhibits contraction. (Wissmann et al., 1997; Wissmann et al., 1999) (Figure 11). The RhoGAP RGA-2 negatively regulates LET-502 in this process (Diogon et al., 2007). RHGF-2 is the corresponding Rho GEF: even though both rga-2 and rhgf-2 alone are lethal, the double mutant is viable (Chan et al., 2015). Interestingly, the Mtl Rac GTPase MIG-2 and the UNC-73 Trio Rac/Rho GEF act in the MEL-11 pathway in this process. UNC-73 Trio acts as a GEF for RHO-1, CED-10, and MIG-2 (Steven et al., 1998; Spencer et al., 2001; Wu et al., 2002). These results indicate that MIG-2 Mtl Rac and RHO-1 might have opposing roles in embryonic elongation (Piekny et al., 2000) (Figure 11). The Rac CED-10, the Rac GEF PIX-1, and PAK-1/PAK regulate muscle hemidesmosome formation, which mediate the latter part of elongation after hypodermal contraction (Zhang et al., 2011). PAK-1 acts in parallel to LET-502 (Gally et al., 2009), and PAK-1 might be regulated by PIX-1 during elongation (Martin et al., 2014). 


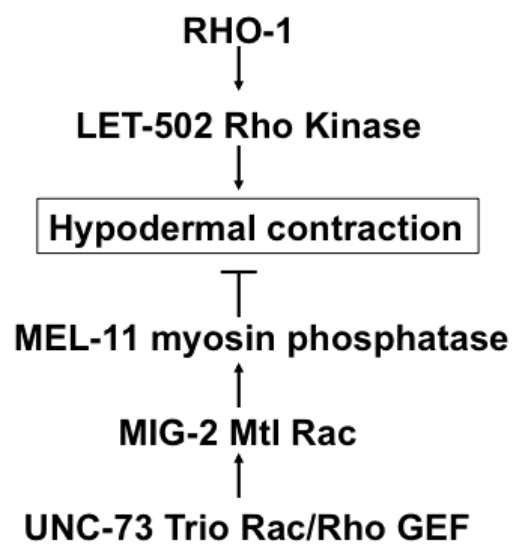

Figure 11. The opposing roles of RHO-1 and MIG-2 Rac in hypodermal contraction during embryonic elongation. Circumferential actin bundles in the hypodermis contract to drive embryonic elongation. RHO-1 stimulates hypodermal contraction via the LET-502 Rho-binding kinase, and MIG-2/Mtl/Rac inhibits hypodermal contraction via MEL-11 myosin phosphatase in response to UNC-73 Trio Rac/Rho GEF.

\subsubsection{P-cell migration}

Later in development, rho- 1 controls the ventral migrations of the P cells (Spencer et al., 2001). The P cell nuclei are born in sublateral locations and migrate ventrally to align at the ventral midline. RHO-1 controls migration of the P cells along with the GEF UNC-73/Trio and LET-502 (Steven et al., 1998; Spencer et al., 2001). Interestingly, the Rac GTPases CED-10 and MIG-2 control P-cell migration in a parallel redundant manner with RHO-1 (Spencer et al., 2001). ECT-2 activation of RHO-1 also controls migration of $\mathrm{P}$ cells as well as in induction of the $1^{\circ}$ vulval cell fate in vulval patterning (Canevascini et al., 2005; Morita et al., 2005; Mitin et al., 2007; Cook et al., 2014).

\subsubsection{Neuronal morphogenesis and function.}

RHO-1 also controls neuronal cell shape after the establishment of the normal axon and dendrite morphology of the neuron. Expression of dominant-negative RHO-1(T19N) in the ASE sensory neuron resulted in an expanded cell body morphology and ectopic neurite initiation (Zallen et al., 2000). This phenotype is similar to that caused by loss of function of the SAX-1 Ndr kinase (Zallen et al., 2000), a known downstream target of Rho activity in other systems. RHO-1 and SAX-1 might act together to regulate neuronal morphogenesis. The RhoGEF RHGF-2 inhibits neurite outgrowth when over-expressed in neuroblastoma cells, similar to Rho constitutive activation (Lin et al., 2012). RHO-1 also regulates neurotransmitter release (Steven et al., 2005; Hiley et al., 2006; McMullan et al., 2006; $\mathrm{Hu}$ et al., 2011). The RhoGEF OSG-1 sensitizes neurons to oxidative stress, which might be part of the aging process (Duan and Sesti, 2015). RHO-1 may also cooperate with a non-canonical LET-60/Ras function in response of the rectal epithelium to pathogen (McMullan et al., 2012).

\subsection{CDC-42}

CDC-42 is required for an array of developmental events involving the cytoskeleton, cell polarity, and protrusion. The roles of CDC-42 in some of these events are described below. Two themes emerge from these studies: a conserved signaling module of CDC-42 with the polarity proteins PAR-3/PAR-6/PKC-3 is iteratively used in multiple developmental events, and CDC-42 acts upstream of the Rac GTPases CED-10 and MIG-2 in protrusive events in a Rho GTPase hierarchy of signaling.

\subsubsection{Embryonic polarity}

$c d c$-42-directed RNAi caused defects in embryonic cytokinesis similar to rho-1(RNAi) (Kay and Hunter, 2001). $c d c-42(R N A i)$ also perturbed the polarity of the single-celled zygote and resulted in defects in anterior-posterior axis formation and mitotic spindle orientation (Gotta et al., 2001; Kay and Hunter, 2001). Upon fertilization, sperm entry results in a rearrangement of the zygote cytoplasm such that anterior cortex accumulates PAR-3 and PAR-6 and the posterior cortex accumulates PAR-2, which together define the anterior-posterior axis of the organism (Levitan et al., 1994; Etemad-Moghadam et al., 1995; Watts et al., 1996; Hung and Kemphues, 1999. The PAR proteins associate with the actin cytoskeleton at the cell cortex, and the actin cytoskeleton is required for their localization. As a result of PAR gene localization, the first cell division generates a larger anterior cell and a 
smaller posterior cell, whose spindle rotates $90^{\circ}$ in relation to the anterior cell (Kemphues et al., 1988). Mutations in the par genes disrupt zygotic polarity and result in the symmetric division of zygote and defects in spindle orientation. RNAi of $c d c-42$ results in a similar loss of zygotic polarity and an associated failure to localize the PAR proteins to their proper domains (Gotta et al., 2001; Kay and Hunter, 2001). $c d c-42$ controls the localization of the PAR proteins via acto-myosin contractility along with RHO-1 (Schonegg and Hyman, 2006). CDC-42 physically associates with PAR-6, a CRIB- and PDZ-domain-containing component of a conserved complex including PAR-3, and PKC-3, a protein kinase $\mathrm{C}$ ortholog, that defines the anterior cortical domain in the zygote (Gotta et al., 2001; Aceto et al., 2006). The CDC-42 GEF CGEF-1 and the CDC-42 GAP CHIN-1 act with CDC-42 in embryonic polarity (Kumfer et al., 2010; Beatty et al., 2013). A conserved CDC-42/PAR-3/PAR-6/PKC-3 module acts in multiple cellular events beyond embryonic polarization, including neuronal morphogenesis and cell migration (Welchman et al., 2007).

As cell division proceeds, blastomeres distinguish between inner surfaces, characterized by cell-cell contact, and outer surfaces, indicated by lack of cell-cell contact. This process is known as radial polarity. CDC-42 activity is inhibited by the RhoGAP PAC-1 at sites of blastomere contact with one another (Anderson et al., 2008). The RhoGEFs CGEF-1 and ECT-2 activate CDC-42 at the cortex of contact-free blastomere surfaces (Chan and Nance, 2013). This results in PAR-6 recruitment to the cortex at no-contact sites, and exclusion from contact sites, to maintain radial polarity.

\subsubsection{Ventral hypodermal enclosure}

During $C$. elegans gastrulation, the dorsally located hypodermal cells migrate toward the ventral midline, where they meet and completely surround the embryo in a process known as ventral enclosure (Sulston et al., 1983; Williams-Masson et al., 1997; Simske and Hardin, 2001). C. elegans WSP-1 is the homolog of the human Wiskott-Aldrich syndrome protein (WASP). WASP is a downstream effector of Cdc42 that activates the Arp2/3 complex, an actin nucleating and branching complex. wsp- 1 mutants display a low-penetrance embryonic lethality due to a failure of migration of the hypodermal cells and ventral enclosure (Withee et al., 2004). The CDC-42 interacting FBAR proteins TOCA-1 and TOCA-2 also regulate embryonic morphogenesis (Giuliani et al., 2009).

\subsubsection{Axon guidance and neuronal morphogenesis}

UNC-6/Netrin and its receptors UNC-40/DCC and UNC-5 are a conserved dorsal-ventral axon guidance system that regulates both attractive guidance ventrally, and repulsive guidance dorsally. In ventrally directed axons attracted to UNC-6/Netrin, UNC-40/DCC promotes neuronal protrusion from cell bodies and growth cones (Norris and Lundquist, 2011; Demarco et al., 2012). CDC-42 is required for UNC-40/DCC-mediated protrusion, and also affects the guidance of axons attracted to UNC-6/Netrin (Demarco et al., 2012). In protrusion, CDC-42 acts upstream of the Rac GEF TIAM-1 and the Rac GTPases CED-10 and MIG-2 (Demarco et al., 2012), which modulate protrusion via the Arp2/3 complex and the Arp2/3 complex activators WAVE- 1 and WSP-1, respectively (Shakir et al., 2008; Norris et al., 2009).

Consistent with a role in neuronal protrusion, constitutive activation of CDC-42 in neurons results in ectopic protrusive structures dependent upon Rac GTPases and the Arp2/3 complex (Alan et al., 2013). PI3 kinase signaling (e.g., AGE-1/PI3K and AKT-1/AKT), and RICT-1/mTORC2 were also required for protrusion, whereas mTORC1 signaling components (DAF-15/RAPTOR and DAF-16/FOXO) were not (Alan et al., 2013). CDC-42 regulation of protrusion utilizes a complex network of signaling pathways involving Rac GTPases as well as PI3K and mTORC2 signaling (Figure 12).

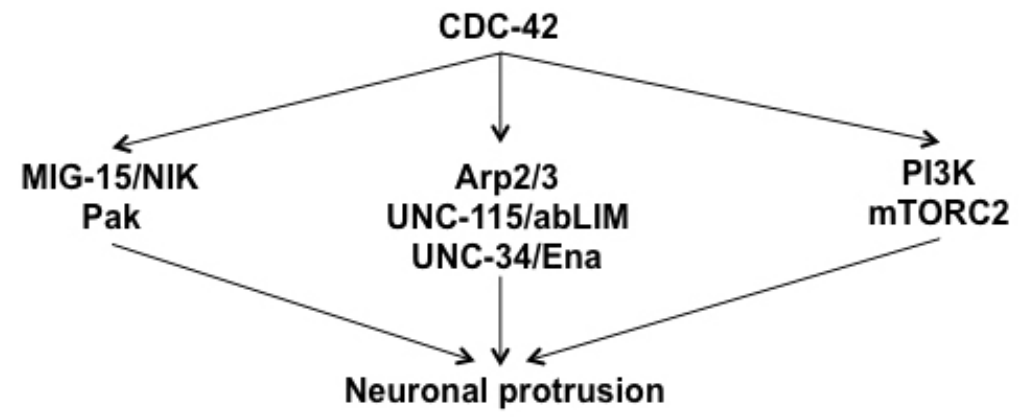

Figure 12. CDC-42 in neuronal protrusion. CDC-42 utilizes multiple pathways to stimulate neuronal protrusion, including the actin-interacting molecules Arp2/3, UNC-34/Enabled, and UNC-115/abLIM, the kinases MIG-15/NIK and PAK-1, and PI3K signaling via the mTORC2 complex. 


\subsubsection{Apoptotic cell corpse engulfment by muscles}

Apoptotic cells are cleared by phagocytosis by neighboring cells. Signals from dying cells induce the formation of phagocytic protrusions from neighboring cells, which involve the Rac GTPases CED-10 and MIG-2 (see Section 3.3). CDC-42 is also required for cell corpse engulfment (Hsieh et al., 2012; Neukomm et al., 2014). In muscles, the $\alpha$ integrin PAT-2 acts with CED-1/CD91, CED-6/GULP, and CED-7/ABC transporter transmembrane protein engulfment module (Neukomm et al., 2014). CDC-42 acts downstream of PAT-2 in engulfment, and upstream of CED-10/Rac (Hsieh et al., 2012; Neukomm et al., 2014). The GEF UIG-1 acts with CDC-42 in engulfment (Hsieh et al., 2012; Neukomm et al., 2014). Interestingly, UIG-1 and CDC-42 are also required for the structure of integrin-containing dense bodies that attach the myofilament lattice to the muscle cell plasma membrane (Hikita et al., 2005).

\subsubsection{Retrograde endocytic recycling}

CDC-42 is involved in trafficking from recycling endosomes to the trans-Golgi network in retrograde endocytic recycling (Bai and Grant, 2015). This also involves the PAR complex (e.g., PAR-6 and PKC-3) and TOCA-1,2 as well as the Arp2/3 activator WVE-1. Components of Wnt secretion utilize this pathway, and thus CDC-42, can affect Wnt signaling via retrograde endocytic recycling (Bai and Grant, 2015).

\subsubsection{Notch signaling}

LIN-12/Notch is necessary and sufficient for $2^{\circ}$ cell fate specification during developmental patterning of the vulva. Genetically, CDC-42 acts as a negative regulator of LIN-12 function in VPC fate specification (Choi et al., 2010), although the molecular nature of this interaction is unclear.

\subsection{Rac-like GTPases CED-10 and MIG-2}

ced-10 encodes a Rac1-like molecule, and mig-2 encodes an Mtl Rac (Zipkin et al., 1997; Reddien and Horvitz, 2000; Lundquist et al., 2001). MIG-2 has similarity to both Cdc42 and Rac molecules, and has an $\mathrm{N}$-terminal predicted myristoylation sequence not found in Cdc42 or Rac. In cell corpse engulfment, MIG-2 is the functional equivalent of vertebrate RhoG (deBakker et al., 2004). ced-10 mutations were identified in mutant screens for defective apoptotic cell corpse phagocytosis (Ellis et al., 1991), and mig-2 mutations were identified in screens for cell migration-defective mutants (Zipkin et al., 1997). The rac-2/3?locus is an apparent tandem duplication, and two deletions ( $o k 326$ and $g k 281$ ) each affect only one copy. rac-2/3 loss-of-function induced by RNAi had no apparent phenotypic consequence (Lundquist et al., 2001; Struckhoff and Lundquist, 2003). It is possible that the $\mathrm{rac}-2 / 3$ locus is non-functional. However, rac-2/3 RNAi did enhance ced-10 and mig-2, suggesting it might have some function. This section will focus on the roles of CED-10 and MIG-2. While ced-10 and mig-2 mutations alone affect some developmental processes (e.g., phagocytosis and cell migration), analysis of pairwise Rac double mutant combinations revealed processes in which ced-10 and mig-2 have overlapping and compensatory roles (i.e., in which they act redundantly). Therefore, the roles of MIG-2 and CED-10 will be discussed together.

\subsubsection{Cell corpse phagocytosis}

ced-10 mutations alone cause failure of phagocytosis of cell corpses after programmed cell death (Ellis et al., 1991; Reddien and Horvitz, 2000). Upon programmed death of a cell, neighboring cells extend actin-based plasma membrane protrusions to engulf and metabolize the cell corpse. ced-10 mutants fail in this process, resulting in the persistence of unengulfed cell corpses. ced-10 might be required to organize the actin cytoskeleton underlying the extension of cellular protrusions of the engulfing cell. Other engulfment mutations identified the ced-1, ced-2, ced-5, ced-6, ced-7, and ced-12 genes (Ellis et al., 1991). These genes can be placed into two pathways based upon redundancy of function in engulfment: ced-1, -6 , and -7 function in parallel to ced-2, $-5,-10$, and -12 . Double mutant combinations between groups cause enhanced engulfment defects, whereas double mutant combinations within each group do not enhance. This observation suggests that two parallel pathways exist to control phagocytosis, one of which involves ced-10 Rac.

Molecular and genetic analyses revealed other CED-10 pathway members in phagocytosis (Figure 13), including CED-2, a CrkII-like receptor adapter protein (Reddien and Horvitz, 2000), and CED-5, a DOCK/CDM-family Rac GEF (Wu and Horvitz, 1998). Furthermore, CED-12 is an ELMO-like protein, which cooperates with CDM proteins in Rac GTP exchange (Gumienny et al., 2001; Zhou et al., 2001; Lu et al., 2004). Indeed, in vertebrate cells and C. elegans, MIG-2 and its exchange factor UNC-73/Trio act upstream of 
CED-5, which in turn activate CED-10 (deBakker et al., 2004). The CED-10/Rac GAP SRGP-1 negatively regulates CED-10/Rac in engulfment (Neukomm et al., 2011), and PDR-1/Parkin negatively regulates CED-10/Rac in phagocytosis possibly via ubiquitylation and targeting CED-10 for degradation (Cabello et al., 2014).

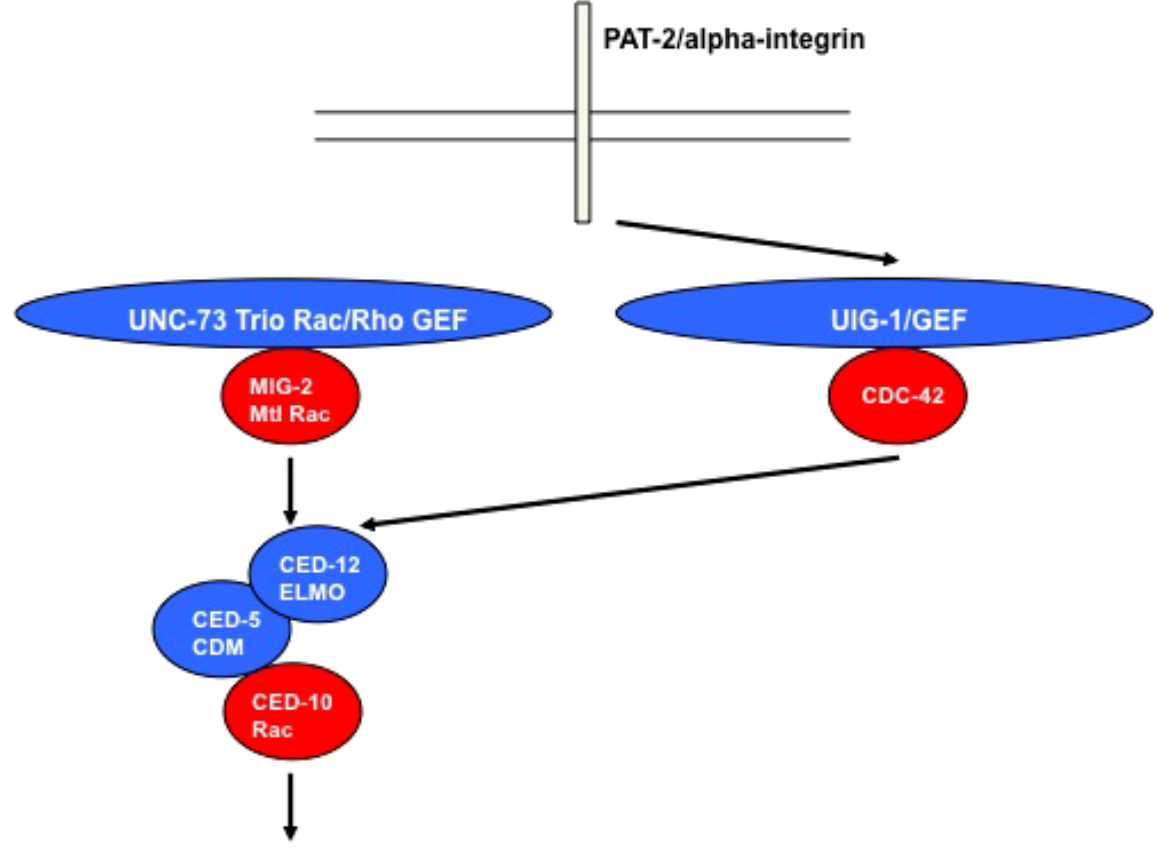

\section{Cell corpse engulfment}

Figure 13. Rho GTPases in programmed cell death corpse engulfment by muscles. CED-10/Rac acts downstream of CDC-42 and the alpha-integrin PAT-2 in extension of cell protrusions in corpse engulfment. MIG-2/RhoG also acts upstream of CED-10/Rac along with the UNC-73/Trio GEF. This pathway likely acts in parallel to the CED-1/6/7 complex.

The CED-1/(CD91/LRP/SREC)/CED-6/(hCED-6/GULP)/CED-7(ABCA1) complex acts in parallel to the CED-10 pathway to regulate actin dynamics underlying phagocytic protrusion (Kinchen et al., 2005). Both pathways might converge on the nucleoside diphosphate kinase NDK-1 in this process (Fancsalszky et al., 2014). A third parallel pathway involves the ABL-1/Abl tyrosine kinase (Hurwitza et al., 2009), illustrating the complex signaling network used to regulate protrusion during phagocytosis.

\subsubsection{Cell migration}

As key regulators of cytoskeletal dynamics and protrusion, ced-10 and mig-2 mutations affect the migration of the gonadal distal tip cells (DTCs), which results in misshapen gonad arms (Lundquist et al., 2001). The dorsalward migration of the DTCs involve UNC-6/Netrin and the UNC-5 receptor (Su et al., 2000). Many of the molecules that act with CED-10 and MIG-2 in phagocytosis are conserved in DTC migration, including the CED-5/CED-12 module (but not CED-2/CrkII), NDK-1, UNC-73/Trio, and PDK-1/Parkin (Gumienny et al., 2001; Lundquist et al., 2001; Neukomm et al., 2011; Cabello et al., 2014). The p21-activated kinase MAX-2 might act downstream of CED-10 in DTC migration (Lucanic et al., 2006; Lucanic and Cheng, 2008; Peters et al., 2013), and the BED domain-containing protein MIG-39 acts with MIG-2 and CED-10 to halt migration of the DTCs (Kikuchi et al., 2015). Furthermore, CACN-1/Cactin acts with MIG-2 in parallel to CED-10 in DTC migration and cessation of migration (Tannoury et al., 2010).

CED-10 and MIG-2 also regulate the migration of the Q neuroblasts and their descendants, along with the NIK kinase MIG-15 and UNC-34/Enabled (Shakir et al., 2006). MIG-15/NIK controls the direction and ability of the Q neuroblasts to extend protrusions and to migrate (Chapman et al., 2008). CED-10 and MIG-2 and their GEFs have both unique and redundant roles in Q migration. MIG-2 and CDC-42, along with the Rac GEFs UNC-73/Trio and PIX-1/PIX are required for robust protrusions of the Q cells preceding migration, whereas CED-10 is required to limit extent of protrusion, similar to its role in DTC migration (Dyer et al., 2010). Double mutant analysis indicates redundancy between MIG-2 and CED-10 in extension of robust protrusions, and that PIX-1/Pix might act in the CED-10 pathway in parallel to UNC-73/Trio and MIG-2 (Dyer et al., 2010). MIG-2 activity correlates with faster and longer $\mathrm{Q}$ descendant cell migration due to its role in maintaining persistent cell and cytoskeletal 
polarization in the direction of migration ( $\mathrm{Ou}$ and Vale, 2009). The migration of the CAN neurons is also redundantly controlled by MIG-2 and CED-10, along with the GEF UNC-73/Trio (Zipkin et al., 1997; Lundquist et al., 2001).

\subsubsection{Gastrulation and morphogenesis}

Null alleles of ced-10 cause maternal-effect embryonic lethality with defects in early epidermal cell movements in gastrulation, resulting in failure of the endoderm to be surrounded by the ectoderm (the gut on the exterior (Gex) phenotype) (Lundquist et al., 2001; Soto et al., 2002). Mutations in gex-1, gex-2, and gex-3 cause a similar maternal-effect lethal phenotype (Soto et al., 2002). GEX-2 encodes an Sra-1-like molecule, and GEX-3 encodes a Hem2-like molecule. Sra-1 and Hem2, along with Nck, form a complex with the Arp2/3 complex activator WAVE (Eden et al., 2002). WAVE-1/WVE acts with GEX-2 and GEX-3 in epidermal gastrulation movements, likely downstream of CED-10 (Patel et al., 2008). This complex leads to the subcellular enrichment of F-actin at apical or basolateral regions in different epidermal cells (Patel et al., 2008).

CED-10 and MIG-2 act redundantly in multiple aspects of vulval development and morphogenesis. mig-2 and ced-10 single mutant vulvae are largely wild-type, whereas mig-2; ced-10 double loss-of-function mutants display defects in the orientation of asymmetric divisions of the $1^{\circ}$ and $2^{\circ}$ vulval cells, indicating that mig- 2 and ced-10 might redundantly control spindle orientation (Kishore and Sundaram, 2002). mig-2; ced-10 doubles also display a failure in the migrations of $2^{\circ}$ vulval cells toward the $1^{\circ}$ vulval cells to form a functional vulva (Kishore and Sundaram, 2002). CED-10 might act downstream of the Semaphorin guidance cue SMP-1 and its receptor PLX-1/Plexin in parallel to UNC-73/Trio and MIG-2 (Dalpe et al., 2005).

In the male tail, CED-10 and MIG-2 are involved in the positioning of the ray 1 sensillum relative to other ray sensilla. The guidance cues SMP-1/Semaphorin, UNC-6/Netrin, and PVF-1/VEGF interact in ray 1 placement, and PVF-1 inhibits CED-10 but not MIG-2 activity during ray 1 placement (Dalpe et al., 2012; Dalpe et al., 2013). The ray 1 response to SMP-1/PLX-1 signaling is governed by level of MIG-2 and CED-10 activity, whereby the attractive response at normal CED-10 and MIG-2 activity is converted to repulsion by reduced activity (Dalpe et al., 2004).

\subsubsection{Cytokinesis}

RhoA activity in formation and function of the actin contractile ring during cytokinesis is governed by the central spindle and the centralspindin complex of proteins. The Rho GAP CYK-4 is a component of centralspindin, and one of its roles is to inhibit CED-10/Rac activity during cytokinesis (Canman et al., 2008). This model is consistent with a general theme of opposing roles of Rho and Rac in the cell, with Rho controlling cell contraction and retraction, and Rac controlling cell protrusion and migration (Burridge and Doughman, 2006).

\subsubsection{Endosomal recycling and apical-basolateral polarity}

Recycling endosomes are critical for maintenance of apico-basolateral polarity in intestinal epithelial cells. In the absence of the 14-3-3 scaffolding protein PAR-5, apical recycling endosomes ectopically accumulate at basolateral positions dependent upon CED-10 and RHO-1 (Winter et al., 2012). The CED-5/CED-12/CED-10 regulatory module also promotes progression of basal recycling endosomes to the plasma membrane by downregulating the RAB-5 GTPase (see Section 4.4) and allowing exit from the early endosome (Sun et al., 2012).

\subsubsection{Synaptic vesicle clustering}

The conserved CED-5/CED-12/CED-10 complex is required for the clustering of synaptic vesicles at active zones in response to the guidance cue UNC-6/Netrin and its receptor UNC-40/DCC (Stavoe and Colon-Ramos, 2012). Active zone positioning itself was not affected by CED-10. The functional consequence of aberrant synaptic vesicle clustering was revealed by pharmacological studies in ced-10 mutants (sensitivity to the GABA(A) receptor antagonist pentylenetetrazole, and to the acetylcholineesterase inhibitor aldicarb) (Locke et al., 2009).

\subsubsection{Wnt signaling}

CED-10/Rac participates in Wnt-dependent developmental events that regulate polarity and the cytoskeleton (Cabello et al., 2010). The CED-5/CED-12/CED-10 regulatory module acts downstream of the Wnt receptor MOM-5/Frizzled, GSK-3 kinase, and APR-1/APC in cell corpse engulfment and DTC migration (Cabello et al., 2010). In orientation of mitotic spindle polarity in the early embryo, MOM-5, GSK-3, and WRM- $1 / \beta-$ catenin regulate $\mathrm{CED}-10 / \mathrm{Rac}$ independently of downstream canonical transcriptional components LIT-1/NLK and POP-1/TCF (Cabello et al., 2010). 


\subsubsection{Axon pathfinding}

ced-10 and mig-2 act redundantly in the pathfinding of axons (Lundquist et al., 2001; Wu et al., 2002). Single mutants display weak defects, which become synergistically stronger in the double mutant. Axon pathfinding in the dorsal, ventral, anterior, and posterior directions are affected, suggesting that CED-10 and MIG-2 act with multiple guidance cues. The double mutant displayed defects in axon outgrowth, axon guidance, and axon branching, indicating that Racs control multiple aspects of axon pathfinding. Furthermore, Rac double mutants displayed ectopic axon branches, suggesting that Racs also control the pruning of spurious axon branches (Struckhoff and Lundquist, 2003). Constitutively active CED-10 and MIG-2 perturb axon pathfinding (Zipkin et al., 1997; Struckhoff and Lundquist, 2003; Shakir et al., 2006; Shakir et al., 2008). Rac(G12V) molecules induce the formation of ectopic lamellipodia and filopodia on neurons that resemble those found on the growth cone during axon outgrowth (Knobel et al., 1999).

Genetic and epistasis analysis indicated that the GEFs UNC-73/Trio (Lundquist et al., 2001; Wu et al., 2002; Struckhoff and Lundquist, 2003) and TIAM-1 (Demarco et al., 2012) act with CED-10 and MIG-2 in axon pathfinding (Figure 14). CED-5/CDM also acts with CED-10 and MIG-2 (Lundquist et al., 2001), suggesting that the conserved CED-5/CED-12 GEF is involved. Downstream, the Arp2/3 activators WVE-1/WAVE and WSP-1/WASP act in the CED-10 and MIG-2 pathways, respectively. WAVE molecules are known to act with Racs. WASP molecules were shown to act with CDC-42, and this work illustrates an interaction of WSP-1 in the MIG-2 pathway. This interaction was confirmed in polarization and intercalation of embryonic epithelial cells, where WSP-1 also acts with MIG-2 (Walck-Shannon et al., 2015). The WASP and WAVE regulators GEX-2/Sra-1 and GEX-3/Hem2 act in both pathways (Shakir et al., 2008). The actin-binding protein UNC-115/abLIM also acts downstream of CED-10 and MIG-2 in axon pathfinding (Struckhoff and Lundquist, 2003). Thus, CED-10 and MIG-2 might utilize at least two distinct pathways to modulate the growth cone actin cytoskeleton: the Arp2/3 complex and UNC-115/abLIM. Indeed, lamellipodial and filopodial protrusions on growth cones are dependent upon Arp2/3 and UNC-115/abLIM (Norris et al., 2009) (Figure 14). The 7WD-repeat receptor of activated C kinase RACK-1 might mediate CED-10 and MIG-2 interaction with UNC-115/abLIM (Demarco and Lundquist, 2010), and the conserved 7WD-repeat protein SWAN-1 might inhibit CED-10 and MIG-2 activity in axon pathfinding (Yang et al., 2006). In addition to initial axon guidance, CED-10 also mediates axon regrowth after severing (Gabel et al., 2008).

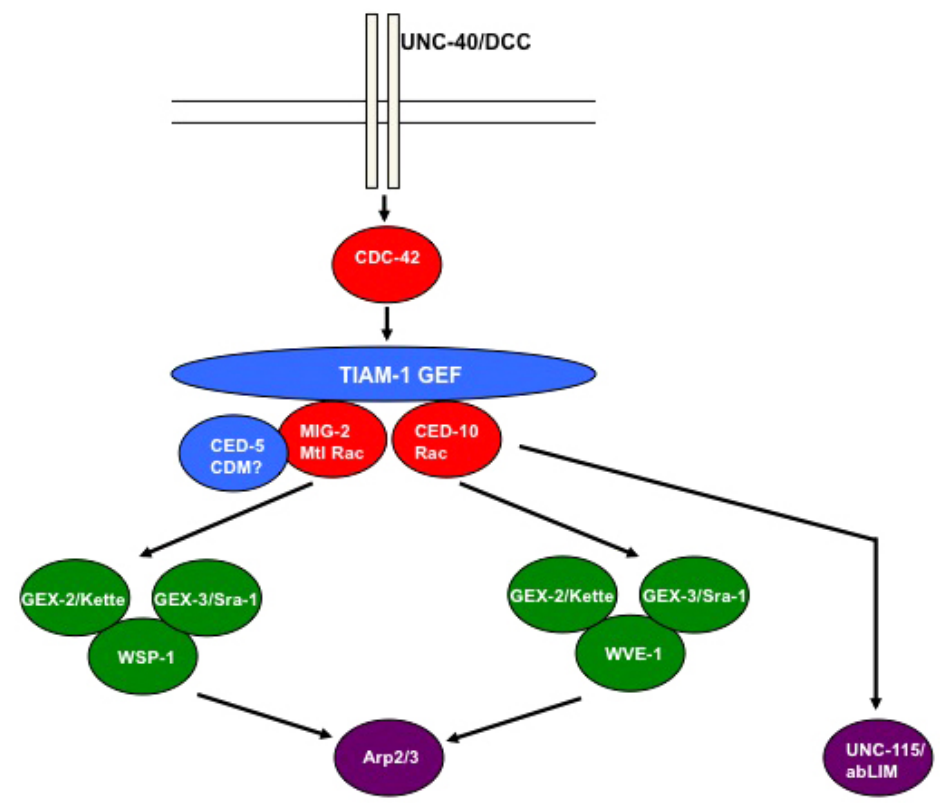

Figure 14. Rho GTPases in attractive axon guidance and neuronal protrusion. CED-10 and MIG- 2 act redundantly downstream of CDC-42 and the guidance receptor UNC-40/DCC in attractive axon guidance and neuronal protrusion. The Arp2/3 activator complex acts in both pathways, with WSP-1/WASP acting in the MIG-2 pathway and WVE-1/WAVE acting in the CED-10 pathway. The GEF TIAM-1 regulates CED-10 and MIG-2 in attractive axon guidance and neuronal protrusion.

Growth cones detect guidance information using transmembrane guidance receptors. Indeed, CED-10 and MIG-2 and the UNC-115 actin-binding protein act downstream of the netrin guidance receptor UNC-40 DCC in 
ventral axon pathfinding (Gitai et al., 2003; Demarco et al., 2012). Furthermore, CED-10, MIG-2, and RAC-2 act with the MIG-15 NIK kinase downstream of the integrin complex in VD/DD motor axon circumferential navigation (Poinat et al., 2002). Asymmetric activation of CED-10 by UNC-6/Netrin signaling results in the asymmetric accumulation of the MIG-10/Lamellipodin that defines axon initiation in attractive guidance (Quinn and Wadsworth, 2008). Furthermore, MIG-2 is inhibited by an UNC-6-independent role of UNC-40/DCC mediated by the SYD-1C RhoGAP-like protein (Xu et al., 2015).

\subsubsection{Growth cone protrusion}

Genetic analyses described above implicate MIG-2 and CED-10 in regulation of axon guidance, and downstream cytoskeletal molecules in growth cone protrusion. Analysis of growth cone protrusive behavior during outgrowth has revealed a role of CED-10 and MIG-2 in regulating protrusion downstream of guidance receptors. The UNC-6/Netrin molecule regulates dorsal-ventral guidance by two distinct receptor complexes: UNC-40/DCC homodimers mediate attractive ventral directed growth, and UNC-5/UNC-40 heterodimers mediate repulsive dorsal growth (Figure 15). Expression of a constitutively activated UNC-40 cytoplasmic domain (MYR::UNC-40) in attracted or repelled neurons had distinct effects. In neurons with axons attracted to UNC-6/Netrin, MYR::UNC-40 led to excessive growth cone protrusion involving lamellipodial and filopodial structures (Gitai et al., 2003; Norris and Lundquist, 2011; Demarco et al., 2012) whereas in repelled growth cones, MYR::UNC-40 inhibited growth cone protrusions (Norris and Lundquist, 2011). These data indicate that the UNC-6/Netrin receptors differentially regulate growth cone protrusion, with the attractive UNC-40/UNC-40 receptor stimulating protrusion, and the repulsive UNC-5/UNC-40 receptor inhibiting protrusion, possibly in a gradient across the growth cone resulting in directed outgrowth.

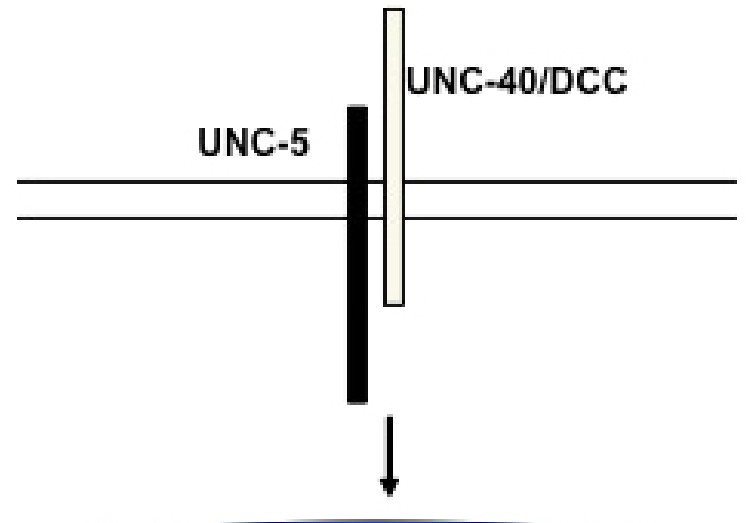

UNC-73/Trio GEF

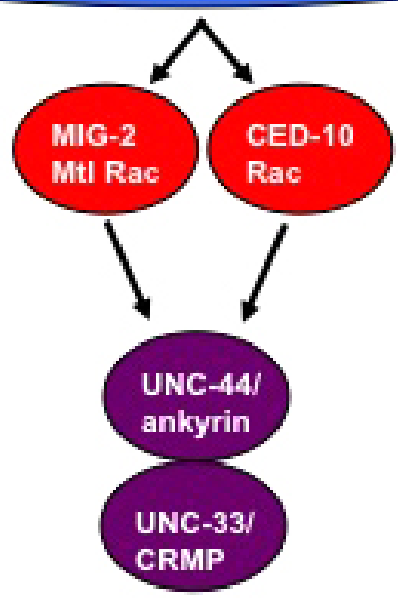

Figure 15. Rac GTPases in repulsive axon guidance and inhibition of growth cone protrusion. Downstream of the repulsive guidance receptor heterodimer of UNC-40/DCC and UNC-5, CED-10 and MIG-2 act redundantly to inhibit growth cone protrusion in repulsive axon guidance via the microtubule-interacting protein UNC-33/CRMP and UNC-44/CRMP. The UNC-73/Trio GEF regulates CED-10 and MIG-2 in inhibition of protrusion. 
CED-10 and MIG-2 were required for ectopic protrusion caused by MYR::UNC-40 in neurons with axons attracted to UNC-6/Netrin (Gitai et al., 2003; Demarco et al., 2012). The GEF UNC-73/Trio was not required, but the GEF TIAM-1 was, indicating that TIAM-1 but not UNC-73 regulate MIG-2 and CED-10 in protrusion. These data along with those discussed above suggest that UNC-40/DCC stimulates growth cone protrusion via TIAM-1, CED-10/MIG-2, and the downstream cytoskeletal effectors UNC-115/abLIM and Arp2/3.

In growth cones repelled from UNC-6/Netrin, MYR::UNC-40 inhibited protrusion, resulting in small growth cones with little of no filopodial protrusions (Norris and Lundquist, 2011; Norris et al., 2014). Consistent with this, loss of repellent UNC-5 receptor resulted in larger-than-normal growth cones with more filopodial protrusion. Using epistasis analysis, a pathway required for growth cone inhibition downstream of UNC-40/UNC-5 repulsive receptor was described, including UNC-73/Trio, MIG-2 and CED-10, and the cytoskeletal regulators UNC-44/Ankyrin and UNC-33/CRMP (collapsin response mediating protein) (Norris et al., 2014) (Figure 15). UNC-33/CRMP is a putative microtubule-interacting protein whose localization is dependent upon UNC-44/Ankyrin (Maniar et al., 2012). Activated CED-10 or MIG-2 expression in these neurons mimicked MYR::UNC-40 and led to small growth cones with few protrusions (Figure 16). UNC-44/Ankyrin and UNC-33/CRMP were required, indicating that they are downstream effectors of CED-10 and MIG-2 (Figure 17). Thus, CED-10 and MIG-2 might inhibit growth cone protrusion downstream of UNC-40/UNC-5 by regulating the microtubule cytoskeleton.
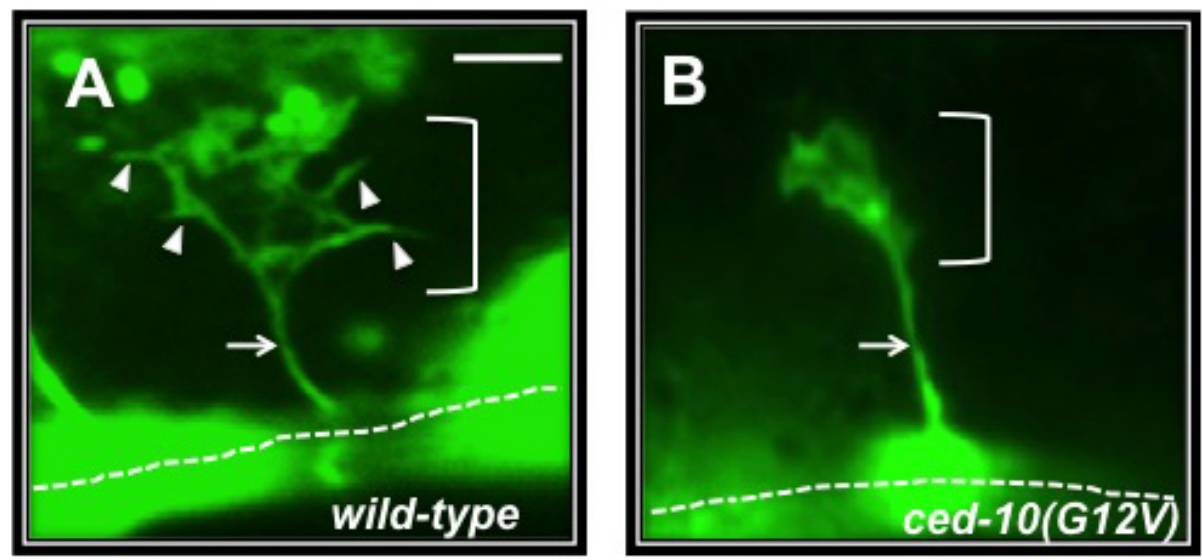

Figure 16. Activated CED-10(G12V) inhibits VD growth cone protrusion. Fluorescent micrographs of Punc-25::gfp expression in the growth cones of VD neurons in early L2 animals are shown. A) A wild-type VD growth cone displayed multiple robust filopodial protrusions. B) A VD growth cone expressing activated CED-10(G12V) displayed no robust filopodial protrusions and was reduced in size relative to the wild type. The scale bar in A represents $1 \mu \mathrm{m}$. Dorsal is up, and anterior is to the right. The ventral nerve cord is indicated by a dashed line. Arrows indicate the VD axon, and the growth cone is bracketed. Arrowheads indicate filopodial protrusions. Micrographs: Mahekta Gujar from the Lundquist lab.

\subsubsection{Modularity of CED-10 and MIG-2 signaling}

In C. elegans, 22 RhoGEFs of the DH-PH family regulate six Rho-family GTPases. Studies of CED-10 and MIG-2 in growth cone protrusion indicate that they can both stimulate and inhibit protrusion (Gitai et al., 2003; Norris and Lundquist, 2011; Demarco et al., 2012; Norris et al., 2014). The data suggest that different modules of CED-10 and MIG-2 associated with distinct GEFs and downstream cytoskeletal effectors mediate these opposing responses (e.g., TIAM-1 and the Arp2/3 complex in stimulation of protrusion, and UNC-73/Trio and UNC-33/CRMP in inhibition) (Figure 17). 


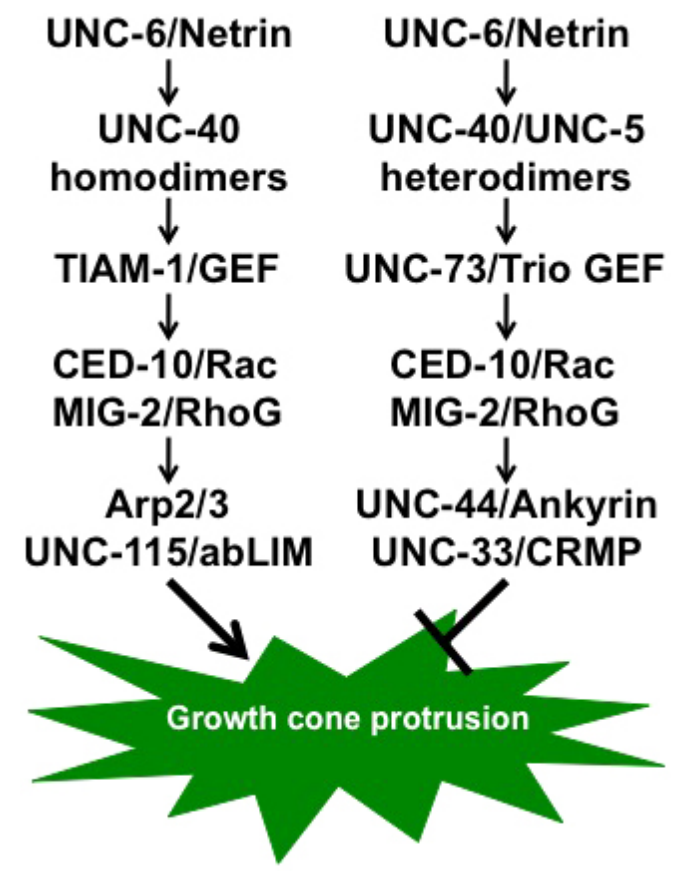

Figure 17. Modularity of Rac signaling in axon pathfinding. Rac GTPases MIG-2 and CED-10 control both stimulation and inhibition of neuronal protrusion in attractive versus repulsive UNC-6/Netrin-mediated axon guidance. The Racs act downstream of distinct receptor complexes (UNC-40 homodimers in attractive/stimulatory, and UNC-40/UNC-5 heterodimers in repulsive/inhibitory). Different GEFs regulate the Racs in each (TIAM-1 in attractive/stimulatory and UNC-73/Trio in repulsive/inhibitory). The stimulatory pathway influences the actin cytoskeleton via Arp/23 and UNC-115/abLIM, and the inhibitory might modulate the microtubule cytoskeleton via UNC-33/CRMP and UNC-44/Ankyrin.

\subsubsection{New, conserved Rho GTPase activating or dominant-negative mutations}

Point mutations that alter GTPase activity or the interactions of Rho GTPases with GEFs, GAPs, and downstream effectors can lead to constitutive activation of the molecules or dominant inactivation of the pathways involving the molecules (e.g., the activating G12 and Q61 mutations found in human tumors, Rac and Ras numbering) (Fernandez-Medarde and Santos, 2011; Forbes et al., 2011). Genetic analysis of C. elegans axon guidance identified new activated and/or dominant-negative mutations in CED-10 and MIG-2 (Shakir et al., 2006). ced-10(lq20)P29L, ced-10(n3246)G60R, and mig-2(lq13)S75F do not behave as loss-of-function mutations and have dominant effects in transgenic expression assays not observed with the wild-type proteins (Shakir et al., 2006). Strikingly, the equivalent P29L mutation in ced-10(lq20) was recently identified in human Racl as a driver of melanoma tumorigenesis (Hodis et al., 2012; Krauthammer et al., 2012), and G60R is synonymous with the let-60(ay75) temperature-sensitive gain-of-function mutation (Schutzman et al., 2001) (see Section 2.1). Biochemical analysis indicated that Rac1P29L was an activating mutation that enhanced interaction with the p21 binding domain (PBD) of PAK kinase. Further analysis will indicate if CED-10(G60R) and MIG-2(S75F) are activating or dominant negative, and if their mechanism is conserved in other organisms including humans.

\subsection{Non-canonical Rho GTPases}

\subsection{1. $\mathrm{CHW}-1$}

chw-1 encodes an ortholog of mammalian proteins Chp/Wrch2/RhoV and Wrch-1/RhoU. Mammalian Wrch-1 was discovered as a gene whose expression is upregulated in response to oncogenic Wnt signaling. Wrch-1 is required for Wnt-dependent transformation and activated Wrch-1 transforms similarly to Wnt (Tao et al., 2001). This subfamily shares most homology with the Cdc42 subfamily. Like Cdc42, Wrch-1 can bind and activate Pak and Par6/aPKC and stimulate JNK (Tao et al., 2001; Chuang et al., 2007; Brady et al., 2009).

The CHW-1 protein structure departs from its Chp/Wrch subfamily members in three ways. First, the position 18 residue (12 by Ras and Rac numbering) is an Ala instead of the Gly that is nearly universal in Ras superfamily proteins. Second, CHW-1 contains a short 7 residue N-terminal extension that contains no prolines, compared to the 45 and 28 residue proline-rich Wrch-1/RhoU and Chp/Wrch-2/RhoV N-terminal extensions, respectively. These Proline-rich N-terminal extensions are thought to mediate auto-inhibitory functions that are relieved by binding of 
SH3 domain-containing proteins like Grb2 (Shutes et al., 2004). The third difference that clearly distinguishes CHW-1 from its subfamily relatives is the lack of a C-terminal membrane-targeting sequence. 3' RACE, RT-PCR, and subsequent RNAseq failed to identify missing 3' coding sequences that could contain the distinctive C-terminal CAAX sequence that mediates lipid modification (D.Reiner, unpublished results). Like many small GTPase C-termini, the CHW-1 C-terminus is enriched with basic residues that could mediate an electrostatic switch, but whether CHW-1 is membrane-targeted like its mammalian relatives is unclear. Notably, all of these CHW-1 sequence features are highly conserved in CHW-1 in related Caenorhabditids C. briggsae and C. remanei.

Animals mutant for the $c h w-1($ ok697) null deletion allele are superficially wild type. This allele also deletes the 5' end of the overlapping gene F22E12.13. A newly identified $c h w-1$ exon 3 (of 4) nonsense allele from the Million Mutation Project (Thompson et al., 2013), gk731043, should disrupt $c h w-1$ function without impacting F22E12.13 function, but has not yet been analyzed. Ectopic expression of wild-type CHW-1 in the distal tip cells of the somatic gonad weakly disrupted DTC migration (Kidd et al., 2015). Similar experiments with wild-type CED-10/Rac and CDC-42 caused no defect on DTC migration, presumably because the activity of most small GTPases are tightly regulated by GEFs and GAPs. Ectopic DTC-specific expression of mutationally activated CED-10/Rac and CDC-42 strongly disrupted DTC migration, consistent with affecting positional information during migration (Peters et al., 2013). Mutating CHW-1 atypical A18 to G (Ras and Rac numbering A12G) abolished migration defects from ectopic expression, while the A18V (Ras and Rac numbering A12V) or Q69L (Ras and Rac numbering Q61L), both predicted to maximally activate CHW-1, increased activation over the wild-type CHW-1 (Kidd et al., 2015). These results suggest that CHW-1 is partially constitutively active, unlike most small GTPases. Genetic experiments suggest that CHW-1 functions non-autonomously in the vulva to equalize contributions of LIN-18/Ryk and LIN-17/Fz receptors in P7.p polarity (Kidd et al., 2015). Thus, like its mammalian counterpart Wrch-1, CHW-1 might act with Wnt signaling.

\subsubsection{CRP-1}

CRP-1 is most similar to CDC-42 (Caruso et al., 2005; Jenna et al., 2005) and has no obvious mammalian counterpart based on sequence, although it remains to be seen if CRP-1 has a functional counterpart in mammals, as does MIG-2 (deBakker et al., 2004). Like other Rho GTPases, CRP-1 has a C-terminal CAAX prenylation sequence but lacks the Rho insert domain found in other Rho GTPases (Jenna et al., 2005). The CRP-1 effector-binding region is highly diverged, suggesting that CRP-1 engages effectors other than canonical Cdc 42 effectors. CRP- 1 also has an unconventional nucleotide-binding P-loop structure, with the highly conserved Gly 12 changed to a Thr in CRP-1. Indeed, CRP-1 is largely deficient in GTP hydrolysis activity, and has a low affinity for GTP (Caruso et al., 2005; Jenna et al., 2005). Thus, while similar to Rho GTPases, GTP hydrolysis and interaction might not be central to CRP-1 function. In fibroblasts, CRP-1 protein localized to the trans-Golgi network and recycling endosome, and affected trafficking in epithelial cells in C. elegans (Jenna et al., 2005).

\section{The Rab family}

As in other systems, Rab GTPases are conserved regulators of multiple aspects of intracellular membrane trafficking and dynamics. The 31 C. elegans Rab-like genes are listed in Table 1 (Section 9) (Gallegos et al., 2012). While genetic analyses in C. elegans have delineated the functions of many of the Rab genes in diverse cellular and developmental events, the roles of others remain mysterious.

Descriptions of the Rab molecules in diverse cellular and developmental events are summarized below. This review is intended as a compendium of the effects of specific rab genes and not a comprehensive review of the processes affected by Rabs in $C$. elegans. Rab molecules in C. elegans with known roles affect membranous organellar formation, maturation, and trafficking, in particular the endocytic and late/recycling endosome (see Wormbook chapter $C$. elegans as a model for membrane traffic). Rabs are involved in clathrin-dependent and independent receptor-mediated endocytosis (RAB-5, 10, and 35), in intra-Golgi and endosome to Golgi retrograde transport (RAB-6.1), recycling from endosomes to the plasma membrane (RAB-10 and RAB-11.1), and maturation and function of the autophagosome in autophagy and the phagosome in cell corpse degradation (RAB-2, 7, and 14) (see Wormbook chapters Autophagy in C. elegans and Programmed cell death). Rabs are also key regulators of lysosomal biogenesis (RAB-6.1 and GLO-1). In the nervous system, Rabs are involved in synaptic vesicle trafficking (RAB-1, 3, 5, 27, and GLO-1), dense core vesicle secretion (RAB-2, 10, and 27), and post-synaptic neurotransmitter receptor recycling via the endocytic pathway (RAB-6.2 and 10). Some other diverse roles of Rabs involve innate immunity (RAB-1), ethanol sensitivity (RAB-3), cortical granule secretion (RAB-6.1), and sensory cilia development (RAB-8). 
That different Rabs have distinct effects suggest that some specificity in function resides in the Rabs themselves and not their regulators (as is the case with Rho GTPases). There is also likely much redundancy of function among the Rabs that have yet to be described, such as RAB-3 and RAB-27 in synaptic vesicle trafficking (Iwasaki et al., 1997; Iwasaki and Toyonaga, 2000; Mahoney et al., 2006). Additionally Rabs act sequentially, such as RAB-2 and RAB-14 acting together to regulating phagosome maturation and upstream of RAB-7, which mediates fusion with the lysosome (Guo et al., 2010; Guo and Wang, 2010). Rabs also act sequentially with other Rho-family GTPases, such as downstream of CED-10/Rac in endocytic recycling (Sun et al., 2012), and upstream of ARF-6 in endosomal trafficking (Nilsson et al., 2008; Shi and Grant, 2013). These themes of redundancy and sequential activity are described in the vignettes below.

\subsection{RAB-1}

RAB-1 controls trafficking of the GPCR odorant receptor ODR-10 into sensory cilia (Chen et al., 2014) and CAV-1/caveolin trafficking in oocytes (Sato et al., 2006). RAB-1 is involved in innate immunity, which consists of the expression and secretion of anti-microbial peptides, the neuropeptide-like proteins (NLPs), and the caenacins (CNCs) (Couillault et al., 2004). RAB-1 acts with TIR-1, a Toll/IL/IR-domain protein similar to vertebrate SARM, in this process (Couillault et al., 2004).

\subsection{RAB-2/UNC-108}

Mutations in $r a b-2$ were first identified by uncoordinated locomotion, and the locus was called $u n c-108$. Here we will refer to it as $r a b-2$. RAB-2 controls a variety of vesicle trafficking and maturation events in neurons and other cells. In neurons, RAB-2 and the RabGAPs TBC-2 and TBC-8 regulate dense core vesicle maturation and sorting (Sumakovic et al., 2009; Hu et al., 2011; Hannemann et al., 2012; Ailion et al., 2014). RAB-2 regulates post-synaptic endocytosis of the GLR-1 receptor to the recycling endosome, but not the multivesicular body degradation pathway (Chun et al., 2008), and trafficking of the GPCR odorant receptor ODR-10 to sensory cilia (Chen et al., 2014). RAB-2 also controls the maturation and function of the phagosome involved in the degradation of apoptotic cell corpses after phagocytic engulfment (Lu et al., 2008; Mangahas et al., 2008; Guo et al., 2010; Guo and Wang, 2010; Lu et al., 2012).

\subsection{RAB-3}

RAB-3 localizes to synaptic vesicles and controls their trafficking to the presynaptic active zone (Nonet et al., 1997; Mahoney et al., 2006; Gracheva et al., 2008). RAB-27 acts with RAB-3, and both are regulated by the Rab GEF AEX-3 in this process (Iwasaki et al., 1997; Iwasaki and Toyonaga, 2000; Mahoney et al., 2006). UNC-10/Rim might act with RAB-3 to control vesicle fusion (Koushika et al., 2001; Gracheva et al., 2006) and with RAB-3/AEX-3 to mediate UNC-57/Endophilin association with SVs to promote fusion (Bai et al., 2010).

RAB-3 loss results in sensitivity to ethanol and other inhalants (Kapfhamer et al., 2008; Davies et al., 2012), and this effect appears to be independent of RAB-3's role in synaptic vesicle secretion (Johnson et al., 2013). RAB-3 might affect other neurosecretory events such as dense core vesicle secretion, or have some other as-yet unknown role in ethanol sensitivity.

\subsection{RAB-5}

RAB-5 regulates the early to late endosome maturation process along with RAB-7 (Chotard et al., 2010; Poteryaev et al., 2010). In the nervous system, this involves the earliest stages of synaptic vesicle formation (Sann et al., 2012). The GAP TBC-2 negatively regulates RAB-5 and is recruited to the early endosome as it matures, removing RAB-5 to allow later-acting Rabs, such as RAB-7 (degradation) and RAB-10 (recycling), to function. RAB-10 directly recruits TBC-2, with help from CED-10 and AMPH-1 (Chotard et al., 2010; Sasidharan et al., 2012; Sun et al., 2012; Liu and Grant, 2015). In addition, RAB-5 is involved in endocytosis of yolk (Grant and Hirsh, 1999; Balklava et al., 2007), and the GEF RME-6 mediates association with clathrin-coated pits (Sato et al., 2005).

RAB-5 is involved in a number of other trafficking and membrane-associated events. In neurons it is involved in dense core vesicle release along with RAB-10 (Sasidharan et al., 2012) and sensory ciliary transport (Kaplan et al., 2012). RAB-5 is also required for phagosomal maturation involved in degradation of engulfed cell corpses (Li et al., 2009; Kinchen and Ravichandran, 2010; Li et al., 2012). In the early embryo, RAB-5 regulates the association of 
the polarity protein PAR-6 with the cell cortex and affects actin organization (Hyenne et al., 2012). Along with RAB-11, RAB-5 is involved in plasma membrane repair after damage by bacterial pore-forming toxins (Los et al., 2011). RAB-5 also has a role in maintaining the structure of the endoplasmic reticulum, which seems to be independent of its role in endosomal trafficking (Audhya et al., 2007). The RAB-5 GEF, RABX-5, which contains a zinc finger and Vps9 domain, acts with RAB-5 and the RAB-5 effector RABN-5 in multiple events including endosomal trafficking and synaptic vesicle release (Poteryaev et al., 2010; Sann et al., 2012).

\subsection{RAB-6.1}

In the oocyte after fertilization, RAB-6.1 is required for fusion of the cortical granules to the oocyte plasma membrane by recruiting the separase fusion protein to the cortical granules (Kimura and Kimura, 2012). RAB-6.1 associates with VPS-52, a component of the Golgi-associated GARP complex that functions in retrograde transport within the Golgi and in Golgi-to-endosome transport (Luo et al., 2011). rab-6.1; rab-6.2 double knockdown treatment results in sterility, suggesting that these molecules act redundantly in the germline (Audhya et al., 2007).

\subsection{RAB-6.2}

In neurons, RAB-6.2 is involved in the retrograde recycling of the GLR-1 AMPA-type glutamate receptor along with the retromer complex (Zhang et al., 2012). The grinder is an extracellular structure in the pharynx required to crush bacteria as they are eaten. RAB-6.2 and the GAP EAT-17 are required for secretion and formation of the pharyngeal grinder (Straud et al., 2013).

\subsection{RAB-7}

RAB-7 controls late endosomal maturation along with RAB-5 (Poulin and Ahringer, 2005; Poteryaev et al., 2007; Sato et al., 2008; Chotard et al., 2010). In autophagy, RAB-7 controls the maturation of the autophagosome (Jenzer et al., 2014; Manil-Segalen et al., 2014) and the phagosome in cell corpse degradation (Kinchen et al., 2008; Yu et al., 2008; Li et al., 2009; Guo et al., 2010; Guo and Wang, 2010; He et al., 2010; Lu et al., 2011) along with CED-10/Rac (Nieto et al., 2010). Other trafficking events controlled by RAB-7 include formation of gut lysosomes (Delahaye et al., 2014), and late endosomal trafficking that antagonizes let-23/EGFR signaling in the vulva (Skorobogata and Rocheleau, 2012). RAB-7 might also play a role in $\alpha$-synuclein lethality in a transgenic model of $\alpha$-synuclein overexpression (Kuwahara et al., 2008).

\subsection{RAB-8}

In ciliated sensory neurons, RAB- 8 is required for membrane delivery to ciliary and periciliary domains, balanced against clathrin and dynamin-mediated removal of material from these domains. (Mukhopadhyay et al., 2008; Kaplan et al., 2010; Kaplan et al., 2012). Maintenance of ciliary and periciliary domains is disrupted in Bardet-Biedl syndrome (Avidor-Reiss and Leroux, 2015).

\section{9. $R A B-10$}

RAB-10 is generally involved in endocytic recycling. It regulates endosomal phosphatidylinositol-4,5-bisphosphate (PI(4,5)P2) levels, and via the Arf GAP CNT-1, downregulates the small GTPase ARF-6, allowing interaction of a series of other downstream effectors involved in vesicle budding (e.g., RME-1 and SPDN-1) (Nilsson et al., 2008; Shi and Grant, 2013). It acts with exocyst in endocytic regulation (Jiu et al., 2012), as well as with actin-binding protein EHBP-1 and NUM-1/Numb in endosomal transport in intestine (Nilsson et al., 2008; Shi et al., 2010). NUM-1 acts between RAB-10 and RME-1 but is not a direct target of RAB-10 as is EHBP-1. A clathrin-independent role of RAB-10 is involved in the recycling of basolateral regions via a novel endosomal compartment in intestinal cells (Chen et al., 2006; Shi et al., 2010; Chen et al., 2014). In neurons, RAB-10 regulates dense core vesicle secretion along with RAB-5 (Sasidharan et al., 2012) and glutamate receptor recycling in post-synaptic regions along with NUM-1/Numb (Glodowski et al., 2007).

\subsection{RAB-11.1}

In epithelial cells RAB-11.1 is positioned by the polarity molecule PAR-5 to mediate apical-basolateral polarity (Winter et al., 2012). RAB-11.1 vesicles localize to the cleavage furrow and mid-body during cell division downstream of separase and the receptor for activated C kinase RACK-1 (Ai et al., 2009; Bembenek et al., 2010), necessary for proper spindle alignment (Zhang et al., 2008). After fertilization, RAB-11.1 is required for secretion of chondroitin proteoglycans (Sato et al., 2008). 


\subsection{RAB-14}

In engulfment of cell corpses, RAB-14 controls phagosome maturation along with RAB-7 and RAB-2/UNC-108 (Guo et al., 2010; Guo and Wang, 2010). RAB-14 and RAB-2/UNC-108 act redundantly in phagosome maturation, and RAB-7 mediates fusion with the lysosome in a sequential action of Rab GTPases in this process.

\subsection{AEX-6}

AEX-6 was identified as a mutant with defects in defecation, and was later found to regulate neuromuscular synaptic transmission underlying defecation and to correspond to RAB-27. AEX-6 is controlled by the AEX-3 GEF and the effector RBF-1/Rabphilin might act downstream to modulate SNARE function in synaptic vesicle release (Mahoney et al., 2006; Tanaka et al., 2008). AEX-6 also has a role in dense core vesicle release (Feng et al., 2012) and possibly insulin secretion involved in aging and Dauer larva formation (Ailion and Thomas, 2003).

\subsection{GLO-1}

GLO-1 Rab GTPase is required for lysosomal organization in the formation of gut granules-birefringent lysosome associated organelles - in the intestine (Hermann et al., 2005; Delahaye et al., 2014). Furthermore, GLO-1 is required downstream of RPM-1/PHR in synaptic organization and axon termination. The RCC GEF GLO-4 regulates GLO-1 in this process, which likely involves a late endosomal pathway that acts in parallel to FSN-1, which acts with RPM-1 in the E3 ubiquitin ligase pathway (Grill et al., 2007).

\subsection{RAB-35}

RAB-35 controls accumulation of yolk receptors at the cortex of oocytes, which are required for clathrin-based receptor-mediated endocytosis of yolk proteins (Sato et al., 2008). RME-4 is a DENN-domain containing Rab GEF that might link RAB-35 to clathrin-coated pits. This complex might act upstream of RAB-7 and synergistically with RAB-11.

\subsection{RAB-39}

RAB-39, along with its binding partner RASF-1/RASSF protect against oxidative stress (Takenaka et al., 2013). RASF-1 interacts specifically with the GTP-bound form of RAB-39, but the basis of resistance to oxidative stress mediated by this complex is unclear.

\subsection{RSEF-1}

RSEF-1 is a divergent Rab-like GTPase similar to RASEF, with a calcium-binding EF hand domain in addition to the GTPase domain. RSEF-1 is required for the development of the uterine seam, a syncytial structure that connects the uterus to the body wall of the animal (Ghosh and Sternberg, 2014). RSEF-1 affects nuclear migration and process outgrowth in the uterine seam.

\section{The Arf/Sar family}

The $C$. elegans genome encodes 14 proteins in the Arf family. The Arf (ADP-ribosylation factor) family gets its name from their ability to stimulate ADP-ribosylation of $\mathrm{G} \alpha$ subunits of heterotrimeric $\mathrm{G}$ proteins in collaboration with cholera toxin; ADP-ribosylation is a form of post-translational modification of proteins and is also the mechanism of action of various bacterial toxins. Arfs are generally involved in membrane/vesicle trafficking and cytoskeletal dynamics. Notably, Arfs are generally lipid modified by a N-terminal myristoyl group rather than the C-terminal farnesyl, geranylgeranyl, or palmitoyl modification found in most Ras superfamily members. Like other members of the Ras superfamily, Arfs are tightly regulated by GEFs and GAPs and have characterized effectors in various systems. Based on sequence SAR1 is considered to be part of the Arf group. Arls are typically similar to Arfs, but their functions are broader (Donaldson and Jackson, 2011).

The Arf family is divided into three classes: the Arfs, the Arf-like proteins (Arls), and Sar1. C. elegans expresses a single Class I Arf, ARF-1.2 (human Arf1 and Arf3), a single Class II Arf, ARF-3 (human Arf4 and Arf5), and a single class III Arf (human Arf6). ARF-1.1 is an outlier with no clear membership in a particular class, and may be a $C$. elegans-specific Arf. ARL-1 is the Arl protein most closely related to the Arf subfamily (Skorobogata et al., 2014). 


\subsection{ARF-1.1}

arf-1.1 encodes an Arf-like gene that does not fall into a specific class or align with a specific canonical Arf type. ARF-1.1 proteins can be found in Caenorhabditids but not more distant nematode relatives. ARF-1.1 has been shown to repress meiotic maturation (Govindan et al., 2006).

\subsection{ARF-1.2}

arf-1.2 encodes an ARF1/3 ortholog required for embryonic development and proper secretion in multiple tissues. ARF-1.2 is broadly expressed throughout development, particularly in embryos. ARF1.2 functions in endoplasmic reticulum dynamics, endocytic sorting of proteins and cleavage furrow formation (Grant and Hirsh, 1999; Skop et al., 2004; Poteryaev et al., 2005; Sato et al., 2006). ARF-1.2, ARF-3, and ARF-6 may collaborate to regulate asymmetric cell division (Teuliere et al., 2014), caspase-independent cell extrusion (Denning et al., 2012), and microRNA regulation (Parry et al., 2007).

During patterning of the vulva (described in Section 2.1 and Section 2.7), EGFR must be localized to the basolateral plasma membrane of VPCs to be activated by EGF. The LIN-2/-7/-10 complex mediates basolateral localization. In the absence of this complex, or upon deletion of the EGFR C-terminal PDZ domain recognition peptide recognized by the LIN-2/-7/-10, EGFR is mislocalized to the apical surface of VPCs, which abrogates proper signaling (Simske et al., 1996; Kaech et al., 1998; Whitfield et al., 1999).

AGEF-1 is an ArfGEF: genetic and homology data are consistent with AGEF-1 promoting exchange on ARF-1.2 and ARF-3. AGEF-1, ARF-1.2, and ARF-3 antagonize EGFR signaling, perhaps at the level of re-localizing EGFR to the apical membrane ( Skorobogata et al., 2014). A hypomorphic mutation in agef-1 suppresses a lin-2 mutation, thus blocking the re-localization of LET-23/EGFR to the apical plasma membrane compartment. AGEF-1 also impacts secretion from other cell types and, unsurprising for a key trafficking protein, is an essential gene. Arfs are involved in recruiting clathrin-coated vesicles (Stamnes and Rothman, 1993; Traub et al., 1993; Robinson, 2004). The AP-1 clathrin adaptor complex plays a role in LET-23 localization (Lee et al., 1994; Shim et al., 2000). AGEF-1, ARF1.2, and ARF-3 may function together to localize LET-23/EGFR to the apical membrane (Skorobogata et al., 2014).

CNT-2/AGAP, an ArfGAP containing a G-protein-like domain, Ankyrin-like repeat, and a PH domain, regulates apoptotic fate and daughter size from the Q.p neuroblast asymmetric division (Singhvi et al., 2011). CNT-2 functions in endocytosis. ARF-1.2 (formerly ARF-1) functions in the same process, and arf-1.2(RNAi) suppressed the CNT-2/AGAP mutant phenotype, consistent with CNT-2/AGAP GAP activity directly inhibiting ARF1.2. The putative ArfGEFs, GRP-1/cytohesin, EFA-6, and BRIS-1, also regulate Q neuroblast asymmetric division (Teuliere et al., 2014).

\subsection{ARF-3}

ARF-3 expression specifically in touch receptor neurons is under transcriptional control of MEC-3 (Zhang et al., 2002), though ARF-3 is probably ubiquitously expressed. The joint interactions of multiple Arf family members suggests functional redundancy between them, or multiple layers of vesicle regulation requirements in many cell biological events. Also see joint role of ARF-3 with ARF-1.2 in inhibiting EGFR signaling (in Section 5.2).

\subsection{ARF-6}

ARF-6 is strongly expressed in coelomocytes and generally expressed elsewhere. ARF-6 co-localizes with MTM-6/myotubularin and they function together in an endocytic pathway and regulation of phosphatidylinositol regulation (Dang et al., 2004). The ArfGAP CNT-1/ACAP, in mammals an Arf6-specific GAP, is a binding partner of RAB-10. In the intestine, RAB-10 and CNT-1 co-localize with ARF-6 on recycling endosomes to control synthesis of phosphatidylinositol-4,5-bisphosphate [PI(4,5)P2] from phosphatidylinositol-4-phosphate [PI(4)P] by the type I phophatidylinositol-4-phosphate 5 kinase, an ARF-6 effector. RAB-10 thus activates CNT-1/GAP to inhibit ARF-6 dependent trafficking of PI(4,5)P2 to the plasma membrane (Shi et al., 2012).

The ArfGAP CNT-1/ACAP is also cleaved by CED-3/Caspase during apoptosis, and the N-terminal fragment of CNT-1/ACAP blocks Akt activation by binding and sequestering phosphatidylinositol $(3,4,5)$-triphosphate at the plasma membrane, thus removing the Akt pro-survival activity independently of ARF-6 (Nakagawa et al., 2014). 
Also see the joint role of ARF-6 with ARF-1.2 and ARF-3 in inhibiting Q neuroblast asymmetrical division (in Section 5.2).

\subsection{ARFRP1}

Y54E10BR. 2 encodes the C. elegans ortholog of mammalian Arf-related protein 1 (ARFRP1). In mammals ARFRP1 is thought to function in membrane trafficking, but the specifics are not well understood. Phenotypes for Y54E10BR. 2 mutants have not been described.

\subsection{SAR-1}

sar-1 encodes the SAR1 ortholog, which is present in all eukaryotes. SAR1 proteins have conserved functions in formation of COPI and COPII secretory vesicles. Not surprisingly, SAR-1 has come up in several RNAi screens for diverse biological functions, but SAR-1 has not yet been studied in detail. SAR-1 RNAi depletion was found to impact endoplasmic reticulum stress (Caruso et al., 2008), consistent with its described cell biological role in other systems.

\subsection{ARL-1}

arl-1 encodes an Arl1 ortholog. Drosophila Arf27A, an ARL-1-like protein, is required for RNAi silencing, and this observation was validated in C. elegans by manipulating ARL-1 (Saleh et al., 2006). ARL-1 is faintly expressed in the cytoplasm of male tail hypodermal cells before male tail morphogenesis in the L4 stage. ARL-1::GFP becomes punctate during male tail whip retraction, and $\operatorname{arl}-1(R N A i)$ caused defective retraction of the tail whip (Nelson et al., 2011). Expression in male tail neurons persists in adulthood. Male tail ARL-1::GFP expression in neurons is under positive control of DMD-3 and/or MAB-3 transcription factors, but ARL-1::GFP is negatively regulated by DMD-3 and MAB-3 in hyp10. Initial whole-genome RNAi screens suggest that arl-1 is an essential gene.

\subsection{EVL-20}

evl-20, also known as arl-2, encodes an ARL2 ortholog. Mutations in evl-20 disrupt many morphogenetic events, most notably in the vulva, male tail, and gonad. Cytokinesis is also disrupted in the evl-20 mutant (Antoshechkin and Han, 2002). The evl-20 mutant phenotypes were rescued by transgenic expression of human ARL2 under the control of the evl-20 promoter, but not ARL1 or ARL3-7.

Transcriptional and translational GFP fusions were expressed broadly during embryogenesis, particularly in migrating hypodermis, and in neurons throughout life. EVL-20 was expressed strongly in the vulva during vulval morphogenesis in the L4 larva, and also expressed in uterus, spermatheca, distal tip cells, sheath cells of the gonad, and other tissues whose development was disrupted in the mutant. Putative EVL-20 maternal product was detected in early embryonic blastomeres by antibody, and was localized to the cell cortex. Maternal EVL-20 is also required for embryogenesis, suggesting that early events in the evl-20 mutant are maternally rescued. Depletion of maternal EVL-20 also causes catastrophic defects in the mitotic spindle, highly aneuploid cells, and defective cytokinesis (Antoshechkin and Han, 2002).

\subsection{ARL-3}

arl-3 encodes the C. elegans ARL3 ortholog. ARL-3 and ARL-13 coordinate intraflagellar transport (Li et al., 2010). ARL-3 localizes to the ciliated endings of sensory neurons. The arl-3 mutant is superficially wild type and does not impact sensory ending structure or intraflagellar transport markers. Cilia-specific expression of constitutively activated ARL-3 (Q72L, Q61L in Ras numbering) disrupted sensory cilia function. ARL-3 is thought to inhibit ciliogenesis through HDA-6/HDAC6-mediated regulation of microtubule acetylation. The hda-6 mutant rescues the $\operatorname{arl}-13$ sensory neuron defect to the same degree as the $\operatorname{arl}-3$ mutant, and confers a sensory ending defect when over-expressed in ciliated sensory neurons. There is no additivity between $h d a-6$ and arl-3 mutants, suggesting that they function in a linear pathway (Li et al., 2010).

\subsection{ARL-5}

arl-5 encodes the ortholog of mammalian Arl5. Whole-genome RNAi screens determined that ARL-5 is required for viability and fertility, but ARL-5 has not been studied specifically. 


\subsection{ARL-6}

arl-6 encodes an Arl6 ortholog that is a component of cilia and undergoes intraflagellar transport (Fan et al., 2004). Studies of arl-6 in C. elegans represent a novel and powerful application of model organism genetics to human disease. Bardet-Beidl syndromes (BBSs) are multisystemic disorders of blindness, polydactyly, obesity, and cognitive impairment (Katsanis, 2004). Mutations at multiple loci can cause BBS, but the cellular and molecular mechanism of the disease is unclear. Interestingly, all BBS gene homologs in C. elegans are expressed in ciliated neurons ( $\mathrm{Li}$ et al., 2004), indicating that the disease might affect ciliary function. This fact was used to identify an additional human gene responsible for BBS3 (Fan et al., 2004). C. elegans genes expressed in ciliated neurons contain a DAF-19 RFX binding X-box in their promoters (Ansley et al., 2003; Li et al., 2004). All C. elegans genes with X-boxes in their promoters were identified, which included arl-6, along with their counterparts in the human genome. Those human genes that localized to the genetically defined interval of BBS3 were sequenced in BBS3 patients. By this method, human arl-6 was found to harbor mutations in multiple BBS3 families (Fan et al., 2004).

\subsection{ARL-8}

arl-8 encodes the $C$. elegans ortholog of mammalian Arl8. ARL-8 regulates axonal transport of presynaptic vesicle cargo (Klassen et al., 2010). Animals harboring arl-8 mutations show excessive accumulation of synaptic vesicle precursors, suggesting that ARL-8 functions to repress precursor aggregation. Viable reduced-function mutations were isolated in this study. ARL- 8 and a JNK MAP kinase cascade act in opposition during clustering of presynaptic components, with ARL-8 binding directly to the UNC-104/Kif1A kinesin motor protein (Wu et al., 2013).

Maternally provided ARL-8 is required for proper embryogenesis. Lethality was rescued by human Arl8 as well as wild-type and gain-of-function Q75L (Q61L by Ras numbering) in C. elegans ARL-8, but not by putative dominant-negative T34N (T17N by Ras numbering) ARL-8 (Nakae et al., 2010). In coelomocytes ARL-8 is localized primarily to lysosomes, and ARL-8 is involved in endosome-lysosome transport by promoting late endosome-lysosomal fusion. The failure of animals mutant for arl-8 to fuse late endosomes to lysosomes is consistent with defects in transporting endocytosed macromolecules to the lysosome. Consistent with these observations, ARL-8 is involved in phagolysosome formation and is required for efficient removal of apoptotic cells in the germline (Sasaki et al., 2013). As with late endosomes in coelomocytes (Nakae et al., 2010), in arl-8 mutant germline sheath cells phagosomes fail to fuse with lysosomes, consequently delaying degradation of apoptotic cells. ARL-8 physically binds to the VPS-41 component of the fusion complex.

ARL-8 also functions in endogenous RNAi pathways (Fischer et al., 2013). A non-null ARL-8 mutant has decreased 26G siRNAs in ERGO-1 and ALG-3/4 pathways, and thus may function in 26G siRNA biosynthesis in an unknown manner.

\subsection{ARL-13}

arl-13 encodes an Arl13 ortholog required for ciliogenesis. Human ARL13B is mutated in Joubert syndrome (Cantagrel et al., 2008), which is a disorder of cilia function. The Zebrafish scorpion (sco) mutant encodes ARL13b, which is required for cilia formation in the kidney; sco disruption causes cystic kidneys (Duldulao et al., 2009). ARL13b protein is highly enriched in cilia (Cevik et al., 2010).

Mutants for arl-13 have shortened and malformed cilia. ARL-13 coordinates intraflagellar transport (Cevik et al., 2010; Li et al., 2010); in these mutant animals association between the IFT-A and IFT-B complexes is disrupted. RNAi-mediated depletion of arl-3 (see Section 5.9) partially suppresses the arl-13 mutant phenotype by re-establishing IFT-A and -B association, suggesting that ARL-3 and ARL-13 play opposing roles in IFT complex association. ARL-13 is expressed only in ciliated sensory neurons; early studies suggest that ARL-13::GFP is specifically located to the ciliated endings (Blacque et al., 2005; Li et al., 2010), but later analysis suggests specific localization to a small compartment (Brear et al., 2014). Double mutant analysis suggests that $h d a-6$ and arl-3 are antagonistic (Warburton-Pitt et al., 2014): these differences may rely on assay. Cilia-specific expression of constitutively activated ARL-13 caused more severe disruption of sensory cilia function than did the arl-13 loss-of-function mutant.

Mutants for arl-13 also interacted additively with other sensory cilia mutants, such that ciliary neuron structure was worse in double mutants compared to single mutants suggesting the genes act in parallel (Cevik et al., 2010). Sequence analysis suggested that all ARL-13 homologs are palmitoylated, and ARL-13 and human Arl13b both undergo palmitoylation and are located to a proximal ciliary compartment. 
Mutations in arl-13 disrupted localization of the GFP-tagged G-protein coupled receptor STR-163 specifically in the ASK sensory neurons but not other neurons, and did not impact localization of other receptors, suggesting that ARL-13 functions in a highly selective localization scheme (Brear et al., 2014). The C. elegans SUMO-conjugating enzyme UBC-9 interacts with and SUMOylates the C-terminus of ARL-13. Mutant ARL-13 that cannot be SUMOylated can still support ciliogenesis, but cannot localize certain sensory receptors to endings, and sensory functions mediated by those receptors are abrogated (Li et al., 2010). Constitutively SUMOylated ARL-13 performs all ARL-13 functions.

ARL-13 and mammalian ARL13B are localized to the inversinC-like subciliary compartment (Cevik et al., 2013). ARL-13 palmitoylation and the ARL-13 protein sequence motif, RVVP, prevent distal cilium and nuclear localization. ARL-13 and UNC-119 are partially redundant with NPHP-2/InversinC and KLP-11/kinesin for ciliogenesis (Warburton-Pitt et al., 2014), and together they precisely pattern microtubule structures in the cilia.

\subsection{ARC-1}

arc-1 encodes the ortholog of mammalian ARD1/TRIM23. ARC-1 is a member of the Arf-like family of proteins, but it lacks the ability to promote ADP-ribosylation of substrates. ARC-1, and mammalian relatives, contain an N-terminal RING domain, two BBOX's, a BBC superfamily motif, and the C-terminal Arf GTPase domain, so the protein is definitely not a typical Arf family member. Mammalian ARD1 has been shown to cooperate with HIF-1 in hypoxic signaling (Mazure et al., 2004) and in NF- $\mathrm{KB}$ signaling in anti-viral response (Poole et al., 2009; Arimoto et al., 2010). The function or expression pattern of ARC-1 in C. elegans is unknown.

\section{Ran}

Eukaryotes generally encode a single Ran ortholog. The classic function of Ran is to regulate the cycle of nuclear import and export. A gradient of activated Ran is set up by GEFs and GAPs, with elevated Ran•GTP inside the nucleus and elevated Ran•GDP outside the nucleus (Clarke and Zhang, 2008; Cavazza and Vernos, 2015). Upon nuclear envelope breakdown accompanying cell division, a Ran•GTP gradient, driven by high RCC1/RanGEF proximity to chromosomes, promotes and maintains spindle assembly (Kalab et al., 2002; Kalab et al., 2006).

The C. elegans Ran ortholog is ran-1. Consistent with studies in other systems in which Ran controls import/export, nuclear membrane organization, and spindle organization, ran-1(RNAi) embryos display failure of nuclear membrane reassembly after mitosis (Askjaer et al., 2002; Bamba et al., 2002). Additionally, ran-1(RNAi) causes defects in mitotic spindle formation consistent with a defect in kinetochore association with microtubules rather than a defect in microtubule organization per se. RNAi of genes associated with Ran signaling cause similar defects, including the RanGAP/RAN-2, the Ran-binding protein RanBP2/NPP-10, the RanGEF RCC1/RAN-3, and NTF2/RAN-4. ran-1(RNAi) does not impact meiosis.

The Ran system maintenance of import/export, nuclear membrane structure, nuclear pores, and spindle assembly is important for many other processes that intersect with the nuclear membrane (Fernandez and Piano, 2006; Updike and Strome, 2009). Because of its broad impact, the Ran system arises repeatedly in screens for cell biological effects. Yet it is difficult to separate direct from indirect effects of such a fundamental molecular system.

\section{The Rag family}

Rag GTPases are not technically members of the Ras superfamily. While Rags are functionally GTPases, their structure diverges slightly from that of classic Ras superfamily members and they are not membrane-targeted through C-terminal prenylation. Their GEFs and GAPs are also atypical, consisting of the Ragulator complex (GEF) for RagA/B, the GATOR1 RagA/B GAP complex (Jewell et al., 2013; Bar-Peled and Sabatini, 2014), and the Folliculin RagC/D GAP complex (Petit et al., 2013; Tsun et al., 2013). Rags were first discovered in yeast, with $S$. cerevisiae Gtr1 being required for growth (Bun-Ya et al., 1992). We include the Rags because they have emerged as major regulators of TORC1 and anabolic metabolism. Where Rheb (and perhaps Ral) mediate global inputs of nutritional state in TORC1 and anabolism, via growth factor and insulin signals, Rags mediate local inputs into TORC1 at organelles. For example, Rag activity is high at the lysosome when levels of amino acids are elevated (Sancak et al., 2008).

Rag proteins come in two subfamilies: C. elegans RAGA-1 corresponds to mammalian RagA and RagB. RAGC-1 corresponds to mammalian RagC and RagD. Rag proteins heterodimerize. RagA/B $\cdot$ GTP binds with $\mathrm{RagC} / \mathrm{D} \cdot \mathrm{GDP}$ to activate TORC1, while RagA/B・GDP binds with RagC/D•GTP when dissociated from TORC1 (Jewell et al., 2013; Bar-Peled and Sabatini, 2014). 


\subsection{RAGA-1}

RAGA-1 is similar to mammalian RagA and RagB. Loss of RAGA-1 prevents behavioral and neuromuscular decline associated with aging, perhaps through decreased activation of TORC1-dependent anabolism and attendant decreased lifespan and healthspan (Schreiber et al., 2010). Consistent with this model, raga-1 loss ameliorates the life-shortening effect of high concentrations of food.

\subsection{RAGC-1}

Based on mammalian studies, RAGC-1 is expected to be functionally equivalent to RAGA-1. However, RAGC-1 has not been extensively analyzed.

\section{Summary}

In this chapter, we have described the roles of small GTPases in the many aspects of development and cell biology. As molecular switches their roles in specific events are determined by combinations of distinct GEFs and GAPs (e.g., in the case of the relatively few Rho-family GTPases), or proliferation of gene family members (e.g., in the case of the Rab-family). Questions remain about how GTPase activities are localized or restricted to particular subcellular compartments. For example, CED-10/Rac both stimulates and inhibits protrusion in growth cones, with each activity controlled by distinct GEFs. Future studies will unravel the combinations of regulators, GTPases, and their subcellular localizations that result in specific Rho-family GTPase activities.

\section{Table 1. Ras superfamily GTPases in C. elegans.}

\begin{tabular}{|c|c|c|}
\hline C. elegans gene designation ${ }^{1}$ & C. elegans gene name ${ }^{2}$ & Human/mouse orthologs ${ }^{3}$ \\
\hline \multicolumn{3}{|l|}{ Ras family } \\
\hline ZK792.6 & let-60 & $\mathrm{H}, \mathrm{N}, \mathrm{KRas}^{4}$ \\
\hline C44C11.1 & ras-1 & R-Ras, R-Ras2/TC21 \\
\hline F17C8.4 & ras-2 & M-Ras/R-Ras3 \\
\hline C54A12.4 & $d r n-1$ & $\begin{array}{l}\text { Di-Ras1/Rig, Di-Ras2, } \\
\text { Di-Ras3/Noey2/ARHI }^{5}\end{array}$ \\
\hline$C 27 B 7.8$ & rap-1 & Rap1A, B \\
\hline$C 25 D 7.7$ & rap-2 & Rap2A, B, C \\
\hline C08F8.7 & rap-3 & Atypical Rap \\
\hline Y53G8AR.3 & ral-1 & RalA, B \\
\hline F54C8.5 & rheb-1 & Rheb, Rheb2/RhebL1 \\
\hline C14A11.7 & $s s r-2$ & $\begin{array}{c}\text { Dexamethasone-induced Ras-related } \\
\text { protein }\end{array}$ \\
\hline \multicolumn{3}{|l|}{ Rho family } \\
\hline Y51H4A.3 & rho-1 & RhoA, B, C \\
\hline R07G3.1 & $c d c-42$ & Cdc42, TC10/RhoQ, TCL/RhoJ \\
\hline C09G12.8 & ced-10 & Rac1, 2, 3 \\
\hline K03D3.10 & $r a c-2$ & Atypical $\mathrm{Rac}^{6}$ \\
\hline C35C5.4 & mig-2 & $\begin{array}{c}\text { Invertebrate Mtl (MIG-2-like) family, } \\
\text { functional similarity to mammalian } \\
\text { RhoG }\end{array}$ \\
\hline$F 22 E 12.2$ & chw-1 & Chp/Wrch2/RhoV, Wrch1/RhoU \\
\hline Y32F6B.3 & crp-1 & Atypical Cdc42 \\
\hline
\end{tabular}


Small GTPases

\begin{tabular}{|c|c|c|}
\hline C. elegans gene designation ${ }^{1}$ & C. elegans gene name ${ }^{2}$ & Human/mouse orthologs 3 \\
\hline \multicolumn{3}{|l|}{ Rab family } \\
\hline C39F7.4 & rab-1 & Rab1 \\
\hline F53F10.4 & $r a b-2 / u n c-108$ & Rab2 \\
\hline C18A3.6 & $r a b-3$ & Rab3 \\
\hline F26H9.6 & $r a b-5$ & Rab5 \\
\hline F59B2.7 & $r a b-6.1$ & Rab6 \\
\hline$T 25 G 12.4$ & $r a b-6.2$ & Rab6 \\
\hline W03C9.3 & $r a b-7$ & Rab7 \\
\hline D1037.4 & $r a b-8$ & Rab8 \\
\hline$T 23 H 2.5$ & $r a b-10$ & Rab10 \\
\hline F53G12.1 & $r a b-11.1$ & Rab11 \\
\hline W04G5.2 & $r a b-11.2$ & Rab11 \\
\hline K09A9.2 & $r a b-14$ & Rab14 \\
\hline$Y 92 C 3 B .3$ & $r a b-18$ & Rab18 \\
\hline Y62E10A.9 & $r a b-19$ & Rab19 \\
\hline T01B7.3 & $r a b-21$ & Rab21 \\
\hline$Y 87 G 2 A .4$ & $r a b-27$ & Rab27 \\
\hline Y11D7A.4 & $r a b-28$ & Rab28 \\
\hline Y45F3A.2 & $r a b-30$ & Rab30 \\
\hline R07B1.12 & glo-1 & Rab32/38 \\
\hline F43D9.2 & $r a b-33$ & Rab33 \\
\hline Y47D3A.25 & $r a b-35$ & Rab35 \\
\hline W01H2.3 & $r a b-37$ & Rab37 \\
\hline K02E10.1 & & Rab37 \\
\hline C56E6.2 & & Rab6 \\
\hline F11A5.3 & & Rab2 \\
\hline ZK669.5 & & Rab23 \\
\hline F11A5.4 & & Rab2 \\
\hline \multicolumn{3}{|l|}{ Arf family } \\
\hline B0336.2 & $\operatorname{arf}-1.2$ & Arf1 \\
\hline F57H12.1 & $\operatorname{arf}-3$ & Arf5 \\
\hline Y116A8C.12 & arf-6 & Arf6 \\
\hline F45E4.1 & $\operatorname{arf}-1.1$ & Arf1 \\
\hline Y54E10BR.2 & & ARFRP1 \\
\hline ZK180.4 & sar-1 & Sar1A,B \\
\hline F54C9.10 & arl-1 & Arl \\
\hline F22B5.1 & evl-20 & Arf2 \\
\hline F19H8.3 & arl-3 & Arf3 \\
\hline ZK632.8 & arl-5 & Arf8 \\
\hline C38D4.8 & arl-6 & Arf6 \\
\hline Y57G11C.13 & arl-8 & Arl8A,B \\
\hline
\end{tabular}




\begin{tabular}{|c|c|c|}
\hline C. elegans gene designation ${ }^{1}$ & C. elegans gene name ${ }^{2}$ & Human/mouse orthologs 3 \\
\hline$Y 37 E 3.5$ & arl-13 & Arf2 \\
\hline ZK1320.6 & arc-1/arl-4 & TRIM23 \\
\hline \multicolumn{3}{|l|}{ Ran family } \\
\hline$K 01 G 5.4$ & ran-1 & Ran \\
\hline \multirow{2}{*}{\multicolumn{3}{|c|}{$\begin{array}{l}{ }^{1} \text { The } C . \text { elegans gene designation as noted on Wormbase. } \\
{ }^{2} \text { The three-letter } C \text {. elegans gene designation. Some genes have not yet been assigned a three-letter designation. }\end{array}$}} \\
\hline & & \\
\hline \multicolumn{3}{|c|}{${ }^{3}$ Similarities are as noted in Wormbase. } \\
\hline \multicolumn{3}{|c|}{${ }^{4}$ LET-60, by virtue of its polybasic hyper-variable C-terminus, is most similar to the KRas splice variant KRas4B. } \\
\hline \multicolumn{3}{|c|}{$\begin{array}{l}{ }^{5} \text { Assuming longer-than-annotated N-terminus and conserved GTPase domain based on alternative CUG initiator } \\
\text { codons. }\end{array}$} \\
\hline \multicolumn{3}{|c|}{${ }^{6}$ Possibly a pseudogene, based on non-abundant RNAseq reads. } \\
\hline
\end{tabular}

\section{Acknowledgements}

Thanks to N. Rasmussen for vulval images in Figure 6, M. Gujar for the growth cone images in Figure 16, K. Monahan and V. Muñiz-Medina for sharing unpublished results, J. Alan for helpful discussion, and all of the members of the Lundquist and Reiner labs who contributed to studies of small GTPases in development. Work in the Lundquist lab has been supported by NIH grants R01 NS040945 and R21 NS070417 to E.A.L., the Kansas Infrastructure Network for Biomedical Research Excellence (NIH P20 GM103418), and the Center for Molecular Analysis of Disease Pathways (NIH P20 GM103638). Work in the Reiner lab is supported by NIH grant R01 GM085309 to D.J.R.

\section{References}

Abdus-Saboor, I., Mancuso, V.P., Murray, J.I., Palozola, K., Norris, C., Hall, D.H., Howell, K., Huang, K., and Sundaram, M.V. (2011). Notch and Ras promote sequential steps of excretory tube development in C. elegans. Development 138, 3545-3555. Abstract Article

Aceto, D., Beers, M., and Kemphues, K.J. (2006). Interaction of PAR-6 with CDC-42 is required for maintenance but not establishment of PAR asymmetry in C. elegans. Dev. Biol. 299, 386-397. Abstract Article

Ai, E., Poole, D.S., and Skop, A.R. (2009). RACK-1 directs dynactin-dependent RAB-11 endosomal recycling during mitosis in Caenorhabditis elegans. Mol. Biol. Cell 20, 1629-1638. Abstract Article

Ailion, M., Hannemann, M., Dalton, S., Pappas, A., Watanabe, S., Hegermann, J., Liu, Q., Han, H.F., Gu, M., Goulding, M.Q., et al. (2014). Two Rab2 interactors regulate dense-core vesicle maturation. Neuron 82, $167-180$. Abstract Article

Ailion, M., and Thomas, J.H. (2003). Isolation and characterization of high-temperature-induced Dauer formation mutants in Caenorhabditis elegans. Genetics 165, 127-144. Abstract Article

Alan, J.K., Struckhoff, E.C., and Lundquist, E.A. (2013). Multiple cytoskeletal pathways and PI3K signaling mediate CDC-42-induced neuronal protrusion in C. elegans. Small GTPases 4. Abstract Article

Ambrosio, L., Mahowald, A.P., and Perrimon, N. (1989). Requirement of the Drosophila raf homologue for torso function. Nature 342, 288-291. Abstract Article

Anderson, D.C., Gill, J.S., Cinalli, R.M., and Nance, J. (2008). Polarization of the C. elegans embryo by RhoGAP-mediated exclusion of PAR-6 from cell contacts. Science 320, 1771-1774. Abstract Article 
Ansley, S.J., Badano, J.L., Blacque, O.E., Hill, J., Hoskins, B.E., Leitch, C.C., Kim, J.C., Ross, A.J., Eichers, E.R., Teslovich, T.M., et al. (2003). Basal body dysfunction is a likely cause of pleiotropic Bardet-Biedl syndrome. Nature 425, 628-633. Abstract Article

Antoshechkin, I., and Han, M. (2002). The C. elegans evl-20 gene is a homolog of the small GTPase ARL2 and regulates cytoskeleton dynamics during cytokinesis and morphogenesis. Dev. Cell 2, 579-591. Abstract Article

Arimoto, K., Funami, K., Saeki, Y., Tanaka, K., Okawa, K., Takeuchi, O., Akira, S., Murakami, Y., and Shimotohno, K. (2010). Polyubiquitin conjugation to NEMO by triparite motif protein 23 (TRIM23) is critical in antiviral defense. Proc. Natl. Acad. Sci. U. S. A. 107, 15856-15861. Abstract Article

Armenti, S.T., Chan, E., and Nance, J. (2014). Polarized exocyst-mediated vesicle fusion directs intracellular lumenogenesis within the C. elegans excretory cell. Dev. Biol. 394, 110-121. Abstract Article

Aroian, R.V., Koga, M., Mendel, J.E., Ohshima, Y., and Sternberg, P.W. (1990). The let-23 gene necessary for Caenorhabditis elegans vulval induction encodes a tyrosine kinase of the EGF receptor subfamily. Nature 348, 693-699. Abstract Article

Arur, S., Ohmachi, M., Nayak, S., Hayes, M., Miranda, A., Hay, A., Golden, A., and Schedl, T. (2009). Multiple ERK substrates execute single biological processes in Caenorhabditis elegans germ-line development. Proc. Natl. Acad. Sci. U. S. A. 106, 4776-4781. Abstract Article

Ashrafi, K. Obesity and the regulation of fat metabolism (March 9, 2007), WormBook, ed. The C. elegans Research Community, WormBook, doi/10.1895/wormbook.1.130.1, http://www.wormbook.org. Abstract Article

Askjaer, P., Galy, V., Hannak, E., and Mattaj, I.W. (2002). Ran GTPase cycle and importins $\alpha$ and $\beta$ are essential for spindle formation and nuclear envelope assembly in living Caenorhabditis elegans embryos. Mol. Biol. Cell 13, 4355-4370. Abstract Article

Audhya, A., Desai, A., and Oegema, K. (2007). A role for Rab5 in structuring the endoplasmic reticulum. J. Cell Biol. 178, 43-56. Abstract Article

Avidor-Reiss, T., and Leroux, M.R. (2015). Shared and distinct mechanisms of compartmentalized and cytosolic ciliogenesis. Curr. Biol. 25, R1143-1150. Abstract Article

Bai, J., Hu, Z., Dittman, J.S., Pym, E.C., and Kaplan, J.M. (2010). Endophilin functions as a membrane-bending molecule and is delivered to endocytic zones by exocytosis. Cell 143, 430-441. Abstract Article

Bai, Z., and Grant, B.D. (2015). A TOCA/CDC-42/PAR/WAVE functional module required for retrograde endocytic recycling. Proc. Natl. Acad. Sci. U. S. A. 112, E1443-1452. Abstract Article

Balklava, Z., Pant, S., Fares, H., and Grant, B.D. (2007). Genome-wide analysis identifies a general requirement for polarity proteins in endocytic traffic. Nat. Cell Biol. 9, 1066-1073. Abstract Article

Bamba, C., Bobinnec, Y., Fukuda, M., and Nishida, E. (2002). The GTPase Ran regulates chromosome positioning and nuclear envelope assembly in vivo. Curr. Biol. 12, 503-507. Abstract Article

Bar-Peled, L., and Sabatini, D.M. (2014). Regulation of mTORC1 by amino acids. Trends Cell Biol. 24, 400-406. Abstract Article

Barr, F., and Lambright, D.G. (2010). Rab GEFs and GAPs. Curr. Opin. Cell Biol. 22, 461-470. Abstract Article

Beatty, A., Morton, D.G., and Kemphues, K. (2013). PAR-2, LGL-1 and the CDC-42 GAP CHIN-1 act in distinct pathways to maintain polarity in the C. elegans embryo. Development 140, 2005-2014. Abstract Article

Beitel, G.J., Clark, S.G., and Horvitz, H.R. (1990). Caenorhabditis elegans ras gene let-60 acts as a switch in the pathway of vulval induction. Nature 348, 503-509. Abstract Article

Bembenek, J.N., White, J.G., and Zheng, Y. (2010). A role for separase in the regulation of RAB-11-positive vesicles at the cleavage furrow and midbody. Curr. Biol. 20, 259-264. Abstract Article 
Bennett, C.F., and Kaeberlein, M. (2014). The mitochondrial unfolded protein response and increased longevity: cause, consequence, or correlation? Exp. Gerontol. 56, 142-146. Abstract Article

Berset, T., Hoier, E.F., Battu, G., Canevascini, S., and Hajnal, A. (2001). Notch inhibition of RAS signaling through MAP kinase phosphatase LIP-1 during C. elegans vulval development. Science 291, 1055-1058. Abstract Article

Berset, T.A., Hoier, E.F., and Hajnal, A. (2005). The C. elegans homolog of the mammalian tumor suppressor Dep-1/Scc1 inhibits EGFR signaling to regulate binary cell fate decisions. Genes Dev. 19, 1328-1340. Abstract Article

Bivona, T.G., Quatela, S.E., Bodemann, B.O., Ahearn, I.M., Soskis, M.J., Mor, A., Miura, J., Wiener, H.H., Wright, L., Saba, S.G., et al. (2006). PKC regulates a farnesyl-electrostatic switch on K-Ras that promotes its association with Bcl-XL on mitochondria and induces apoptosis. Mol. Cell 21, 481-493. Abstract Article

Blacque, O.E., Perens, E.A., Boroevich, K.A., Inglis, P.N., Li, C., Warner, A., Khattra, J., Holt, R.A., Ou, G., Mah, A.K., et al. (2005). Functional genomics of the cilium, a sensory organelle. Curr. Biol. 15, 935-941. Abstract Article

Bodemann, B.O., Orvedahl, A., Cheng, T., Ram, R.R., Ou, Y.H., Formstecher, E., Maiti, M., Hazelett, C.C., Wauson, E.M., Balakireva, M., et al. (2011). RalB and the exocyst mediate the cellular starvation response by direct activation of autophagosome assembly. Cell 144, 253-267. Abstract Article

Bonfini, L., Karlovich, C.A., Dasgupta, C., and Banerjee, U. (1992). The Son of sevenless gene product: a putative activator of Ras. Science 255, 603-606. Abstract Article

Bos, J.L. (1989). ras oncogenes in human cancer: a review. Cancer Res. 49, 4682-4689. Abstract Article

Bos, J.L., Rehmann, H., and Wittinghofer, A. (2007). GEFs and GAPs: critical elements in the control of small G proteins. Cell 129, 865-877. Abstract Article

Brady, D.C., Alan, J.K., Madigan, J.P., Fanning, A.S., and Cox, A.D. (2009). The transforming Rho family GTPase Wrch-1 disrupts epithelial cell tight junctions and epithelial morphogenesis. Mol. Cell. Biol. $29,1035-1049$. Abstract Article

Braendle, C., and Felix, M.A. (2008). Plasticity and errors of a robust developmental system in different environments. Dev. Cell 15, 714-724. Abstract Article

Brear, A.G., Yoon, J., Wojtyniak, M., and Sengupta, P. (2014). Diverse cell type-specific mechanisms localize G protein-coupled receptors to Caenorhabditis elegans sensory cilia. Genetics 197, 667-684. Abstract Article

Bringmann, H., and Hyman, A.A. (2005). A cytokinesis furrow is positioned by two consecutive signals. Nature 436, 731-734. Abstract Article

Bun-Ya, M., Harashima, S., and Oshima, Y. (1992). Putative GTP-binding protein, Gtr1, associated with the function of the Pho84 inorganic phosphate transporter in Saccharomyces cerevisiae. Mol. Cell. Biol. 12, $2958-2966$. Abstract Article

Bunney, T.D., and Katan, M. (2006). Phospholipase C epsilon: linking second messengers and small GTPases. Trends Cell Biol. 16, 640-648. Abstract Article

Burridge, K., and Doughman, R. (2006). Front and back by Rho and Rac. Nat. Cell Biol. 8, 781-782. Abstract Article

Cabello, J., Neukomm, L.J., Gunesdogan, U., Burkart, K., Charette, S.J., Lochnit, G., Hengartner, M.O., and Schnabel, R. (2010). The Wnt pathway controls cell death engulfment, spindle orientation, and migration through CED-10/Rac. PLoS Biol. 8, e1000297. Abstract Article

Cabello, J., Samann, J., Gomez-Orte, E., Erazo, T., Coppa, A., Pujol, A., Bussing, I., Schulze, B., Lizcano, J.M., Ferrer, I., et al. (2014). PDR-1/hParkin negatively regulates the phagocytosis of apoptotic cell corpses in Caenorhabditis elegans. Cell Death Dis. 5, e1120. Abstract Article 
Camonis, J.H., and White, M.A. (2005). Ral GTPases: corrupting the exocyst in cancer cells. Trends Cell Biol. 15, 327-332. Abstract Article

Canevascini, S., Marti, M., Frohli, E., and Hajnal, A. (2005). The Caenorhabditis elegans homologue of the proto-oncogene ect-2 positively regulates RAS signalling during vulval development. EMBO Rep. 6, 1169-1175. Abstract Article

Canman, J.C., Lewellyn, L., Laband, K., Smerdon, S.J., Desai, A., Bowerman, B., and Oegema, K. (2008). Inhibition of Rac by the GAP activity of centralspindlin is essential for cytokinesis. Science 322, 1543-1546. Abstract Article

Cantagrel, V., Silhavy, J.L., Bielas, S.L., Swistun, D., Marsh, S.E., Bertrand, J.Y., Audollent, S., Attie-Bitach, T., Holden, K.R., Dobyns, W.B., et al. (2008). Mutations in the cilia gene ARL13B lead to the classical form of Joubert syndrome. Am. J. Hum. Genet. 83, 170-179. Abstract Article

Caruso, M.E., Jenna, S., Beaulne, S., Lee, E.H., Bergeron, A., Chauve, C., Roby, P., Rual, J.F., Hill, D.E., Vidal, M., et al. (2005). Biochemical clustering of monomeric GTPases of the Ras superfamily. Mol. Cell. Proteomics 4, 936-944. Abstract Article

Caruso, M.E., Jenna, S., Bouchecareilh, M., Baillie, D.L., Boismenu, D., Halawani, D., Latterich, M., and Chevet, E. (2008). GTPase-mediated regulation of the unfolded protein response in Caenorhabditis elegans is dependent on the AAA+ ATPase CDC-48. Mol. Cell. Biol. 28, 4261-4274. Abstract Article

Casey, P.J., Solski, P.A., Der, C.J., and Buss, J.E. (1989). p21ras is modified by a farnesyl isoprenoid. Proc. Natl. Acad. Sci. U. S. A. 86, 8323-8327. Abstract Article

Cavazza, T., and Vernos, I. (2015). The RanGTP Pathway: from nucleo-cytoplasmic transport to spindle assembly and beyond. Front. Cell Dev. Biol. 3, 82. Abstract Article

Cevik, S., Hori, Y., Kaplan, O.I., Kida, K., Toivenon, T., Foley-Fisher, C., Cottell, D., Katada, T., Kontani, K., and Blacque, O.E. (2010). Joubert syndrome Arl13b functions at ciliary membranes and stabilizes protein transport in Caenorhabditis elegans. J. Cell Biol. 188, 953-969. Abstract Article

Cevik, S., Sanders, A.A., Van Wijk, E., Boldt, K., Clarke, L., van Reeuwijk, J., Hori, Y., Horn, N., Hetterschijt, L., Wdowicz, A., et al. (2013). Active transport and diffusion barriers restrict Joubert Syndrome-associated ARL13B/ARL-13 to an Inv-like ciliary membrane subdomain. PLoS Genet. 9, e1003977. Abstract Article

Chan, B.G., Rocheleau, S.K., Smit, R.B., and Mains, P.E. (2015). The Rho guanine exchange factor RHGF-2 acts through the Rho-binding kinase LET-502 to mediate embryonic elongation in C. elegans. Dev. Biol. 405, 250-259. Abstract Article

Chan, E., and Nance, J. (2013). Mechanisms of CDC-42 activation during contact-induced cell polarization. J. Cell Sci. 126, 1692-1702. Abstract Article

Chandra, A., Grecco, H.E., Pisupati, V., Perera, D., Cassidy, L., Skoulidis, F., Ismail, S.A., Hedberg, C., Hanzal-Bayer, M., Venkitaraman, A.R., et al. (2012). The GDI-like solubilizing factor PDE $\delta$ sustains the spatial organization and signalling of Ras family proteins. Nat. Cell Biol. 14, 148-158. Abstract Article

Chang, C., Hopper, N.A., and Sternberg, P.W. (2000). Caenorhabditis elegans SOS-1 is necessary for multiple RAS-mediated developmental signals. EMBO J. 19, 3283-3294. Abstract Article

Chapman, J.O., Li, H., and Lundquist, E.A. (2008). The MIG-15 NIK kinase acts cell-autonomously in neuroblast polarization and migration in C. elegans. Dev. Biol. 324, 245-257. Abstract Article

Chardin, P., Camonis, J.H., Gale, N.W., van Aelst, L., Schlessinger, J., Wigler, M.H., and Bar-Sagi, D. (1993). Human Sos1: a guanine nucleotide exchange factor for Ras that binds to GRB2. Science 260, 1338-1343. Abstract Article 
Chen, C., Itakura, E., Weber, K.P., Hegde, R.S., and de Bono, M. (2014). An ER complex of ODR-4 and ODR-8/Ufm1 specific protease 2 promotes GPCR maturation by a Ufm1-independent mechanism. PLoS Genet. 10, e1004082. Abstract Article

Chen, C.C., Schweinsberg, P.J., Vashist, S., Mareiniss, D.P., Lambie, E.J., and Grant, B.D. (2006). RAB-10 is required for endocytic recycling in the Caenorhabditis elegans intestine. Mol. Biol. Cell 17, 1286-1297. Abstract Article

Chen, E.B., Branda, C.S., and Stern, M.J. (1997). Genetic enhancers of sem-5 define components of the gonad-independent guidance mechanism controlling sex myoblast migration in Caenorhabditis elegans hermaphrodites. Dev. Biol. 182, 88-100. Abstract Article

Chen, L., Fu, Y., Ren, M., Xiao, B., and Rubin, C.S. (2011). A RasGRP, C. elegans RGEF-1b, couples external stimuli to behavior by activating LET-60 (Ras) in sensory neurons. Neuron 70, 51-65. Abstract Article

Chen, N., and Greenwald, I. (2004). The lateral signal for LIN-12/Notch in C. elegans vulval development comprises redundant secreted and transmembrane DSL proteins. Dev. Cell 6, 183-192. Abstract Article

Chen, S., Li, L., Li, J., Liu, B., Zhu, X., Zheng, L., Zhang, R., and Xu, T. (2014). SEC-10 and RAB-10 coordinate basolateral recycling of clathrin-independent cargo through endosomal tubules in Caenorhabditis elegans. Proc. Natl. Acad. Sci. U. S. A. 111, 15432-15437. Abstract Article

Chen, X.W., Leto, D., Xiong, T., Yu, G., Cheng, A., Decker, S., and Saltiel, A.R. (2011). A Ral GAP complex links PI 3-kinase/Akt signaling to RalA activation in insulin action. Mol. Biol. Cell 22, 141-152. Abstract Article

Chien, Y., Kim, S., Bumeister, R., Loo, Y.M., Kwon, S.W., Johnson, C.L., Balakireva, M.G., Romeo, Y., Kopelovich, L., Gale, M., Jr., et al. (2006). RalB GTPase-mediated activation of the IkB family kinase TBK1 couples innate immune signaling to tumor cell survival. Cell 127, 157-170. Abstract Article

Choi, M.S., Yoo, A.S., and Greenwald, I. (2010). sel-11 and $c d c-42$, two negative modulators of LIN-12/Notch activity in C. elegans. PLoS One 5, e11885. Abstract Article

Chotard, L., Mishra, A.K., Sylvain, M.A., Tuck, S., Lambright, D.G., and Rocheleau, C.E. (2010). TBC-2 regulates RAB-5/RAB-7-mediated endosomal trafficking in Caenorhabditis elegans. Mol. Biol. Cell 21, 2285-2296. Abstract Article

Chuang, Y.Y., Valster, A., Coniglio, S.J., Backer, J.M., and Symons, M. (2007). The atypical Rho family GTPase Wrch-1 regulates focal adhesion formation and cell migration. J. Cell Sci. 120, 1927-1934. Abstract Article

Chun, D.K., McEwen, J.M., Burbea, M., and Kaplan, J.M. (2008). UNC-108/Rab2 regulates postendocytic trafficking in Caenorhabditis elegans. Mol. Biol. Cell 19, 2682-2695. Abstract Article

Clark, S.G., Stern, M.J., and Horvitz, H.R. (1992). C. elegans cell-signalling gene sem-5 encodes a protein with SH2 and SH3 domains. Nature 356, 340-344. Abstract Article

Clarke, P.R., and Zhang, C. (2008). Spatial and temporal coordination of mitosis by Ran GTPase. Nat. Rev. Mol. Cell Biol. 9, 464-477. Abstract Article

Colicelli, J. (2004). Human RAS superfamily proteins and related GTPases. Sci. STKE 2004, RE13. Abstract Article

Conradt, B. and Xue D. Programmed cell death (October 06, 2005), WormBook, ed. The C. elegans Research Community, WormBook, doi/10.1895/wormbook.1.32.1, http://www.wormbook.org. Abstract Article

Cook, D.R., Rossman, K.L., and Der, C.J. (2014). Rho guanine nucleotide exchange factors: regulators of Rho GTPase activity in development and disease. Oncogene 33, 4021-4035. Abstract Article

Cordeddu, V., Di Schiavi, E., Pennacchio, L.A., Ma'ayan, A., Sarkozy, A., Fodale, V., Cecchetti, S., Cardinale, A., Martin, J., Schackwitz, W., et al. (2009). Mutation of SHOC2 promotes aberrant protein N-myristoylation and causes Noonan-like syndrome with loose anagen hair. Nat. Genet. 41, 1022-1026. Abstract Article 
Couillault, C., Pujol, N., Reboul, J., Sabatier, L., Guichou, J.F., Kohara, Y., and Ewbank, J.J. (2004). TLR-independent control of innate immunity in Caenorhabditis elegans by the TIR domain adaptor protein TIR-1, an ortholog of human SARM. Nat. Immunol. 5, 488-494. Abstract Article

Cox, A.D., Der, C.J., and Philips, M.R. (2015). Targeting RAS membrane association: back to the future for anti-RAS drug discovery? Clin. Cancer Res. 21, 1819-1827. Abstract Article

Dalpe, G., Brown, L., and Culotti, J.G. (2005). Vulva morphogenesis involves attraction of plexin 1-expressing primordial vulva cells to semaphorin 1a sequentially expressed at the vulva midline. Development $132,1387-1400$. Abstract Article

Dalpe, G., Tarsitano, M., Persico, M.G., Zheng, H., and Culotti, J. (2013). C. elegans PVF-1 inhibits permissive UNC-40 signalling through CED-10 GTPase to position the male ray 1 sensillum. Development 140, 4020-4030. Abstract Article

Dalpe, G., Zhang, L.W., Zheng, H., and Culotti, J.G. (2004). Conversion of cell movement responses to Semaphorin-1 and Plexin-1 from attraction to repulsion by lowered levels of specific RAC GTPases in C. elegans. Development 131, 2073-2088. Abstract Article

Dalpe, G., Zheng, H., Brown, L., and Culotti, J. (2012). Semaphorin-1 and netrin signal in parallel and permissively to position the male ray 1 sensillum in Caenorhabditis elegans. Genetics 192, 959-971. Abstract Article

Dang, H., Li, Z., Skolnik, E.Y., and Fares, H. (2004). Disease-related myotubularins function in endocytic traffic in Caenorhabditis elegans. Mol. Biol. Cell 15, 189-196. Abstract Article

Daumke, O., Weyand, M., Chakrabarti, P.P., Vetter, I.R., and Wittinghofer, A. (2004). The GTPase-activating protein Rap1GAP uses a catalytic asparagine. Nature 429, 197-201. Abstract

Davies, A.G., Friedberg, R.I., Gupta, H., Chan, C.L., Shelton, K.L., and Bettinger, J.C. (2012). Different genes influence toluene- and ethanol-induced locomotor impairment in C. elegans. Drug Alcohol Depend. 122, $47-54$. Abstract Article

deBakker, C.D., Haney, L.B., Kinchen, J.M., Grimsley, C., Lu, M., Klingele, D., Hsu, P.K., Chou, B.K., Cheng, L.C., Blangy, A., et al. (2004). Phagocytosis of apoptotic cells is regulated by a UNC-73/TRIO-MIG-2/RhoG signaling module and armadillo repeats of CED-12/ELMO. Curr. Biol. 14, 2208-2216. Abstract Article

Delahaye, J.L., Foster, O.K., Vine, A., Saxton, D.S., Curtin, T.P., Somhegyi, H., Salesky, R., and Hermann, G.J. (2014). Caenorhabditis elegans HOPS and CCZ-1 mediate trafficking to lysosome-related organelles independently of RAB-7 and SAND-1. Mol. Biol. Cell 25, 1073-1096. Abstract Article

Demarco, R.S., and Lundquist, E.A. (2010). RACK-1 acts with Rac GTPase signaling and UNC-115/abLIM in Caenorhabditis elegans axon pathfinding and cell migration. PLoS Genet. 6, e1001215. Abstract Article

Demarco, R.S., Struckhoff, E.C., and Lundquist, E.A. (2012). The Rac GTP exchange factor TIAM-1 acts with $\mathrm{CDC}-42$ and the guidance receptor UNC-40/DCC in neuronal protrusion and axon guidance. PLoS Genet. 8, e1002665. Abstract Article

Denning, D.P., Hatch, V., and Horvitz, H.R. (2012). Programmed elimination of cells by caspase-independent cell extrusion in C. elegans. Nature 488, 226-230. Abstract Article

Dibble, C.C., and Cantley, L.C. (2015). Regulation of mTORC1 by PI3K signaling. Trends Cell Biol. 25, $545-555$. Abstract Article

Dickinson, D.J., Pani, A.M., Heppert, J.K., Higgins, C.D., and Goldstein, B. (2015). Streamlined genome engineering with a self-excising drug selection cassette. Genetics 200, 1035-1049. Abstract Article

Diogon, M., Wissler, F., Quintin, S., Nagamatsu, Y., Sookhareea, S., Landmann, F., Hutter, H., Vitale, N., and Labouesse, M. (2007). The RhoGAP RGA-2 and LET-502/ROCK achieve a balance of actomyosin-dependent forces in C. elegans epidermis to control morphogenesis. Development 134, 2469-2479. Abstract Article 
Donaldson, J.G., and Jackson, C.L. (2011). ARF family G proteins and their regulators: roles in membrane transport, development and disease. Nat. Rev. Mol. Cell Biol. 12, 362-375. Abstract Article

Dong, X., Yang, B., Li, Y., Zhong, C., and Ding, J. (2009). Molecular basis of the acceleration of the GDP-GTP exchange of human ras homolog enriched in brain by human translationally controlled tumor protein. J. Biol. Chem. 284, 23754-23764. Abstract

Duan, Z., and Sesti, F. (2015). Guanine nucleotide exchange factor OSG-1 confers functional aging via dysregulated Rho signaling in Caenorhabditis elegans neurons. Genetics 199, 487-496. Abstract Article

Duldulao, N.A., Lee, S., and Sun, Z. (2009). Cilia localization is essential for in vivo functions of the Joubert syndrome protein Arl13b/Scorpion. Development 136, 4033-4042. Abstract Article

Dyer, J.O., Demarco, R.S., and Lundquist, E.A. (2010). Distinct roles of Rac GTPases and the UNC-73/Trio and PIX-1 Rac GTP exchange factors in neuroblast protrusion and migration in C. elegans. Small GTPases 1, 44-61. Abstract Article

Eden, S., Rohatgi, R., Podtelejnikov, A.V., Mann, M., and Kirschner, M.W. (2002). Mechanism of regulation of WAVE1-induced actin nucleation by Rac1 and Nck. Nature 418, 790-793. Abstract Article

Egan, S.E., and Weinberg, R.A. (1993). The pathway to signal achievement. Nature 365, 781-783. Abstract Article

Eisenmann, D.M., and Kim, S.K. (1997). Mechanism of activation of the Caenorhabditis elegans ras homologue let-60 by a novel, temperature-sensitive, gain-of-function mutation. Genetics 146, 553-565. Abstract Article

Ellerbroek, S.M., Wennerberg, K., and Burridge, K. (2003). Serine phosphorylation negatively regulates RhoA in vivo. J. Biol. Chem. 278, 19023-19031. Abstract Article

Ellis, R.E., Jacobson, D.M., and Horvitz, H.R. (1991). Genes required for the engulfment of cell corpses during programmed cell death in Caenorhabditis elegans. Genetics 129, 79-94. Abstract Article

Emkey, R., Freedman, S., and Feig, L.A. (1991). Characterization of a GTPase-activating protein for the Ras-related Ral protein. J. Biol. Chem. 266, 9703-9706. Abstract Article

Etemad-Moghadam, B., Guo, S., and Kemphues, K.J. (1995). Asymmetrically distributed PAR-3 protein contributes to cell polarity and spindle alignment in early C. elegans embryos. Cell 83, 743-752. Abstract Article

Fan, Y., Esmail, M.A., Ansley, S.J., Blacque, O.E., Boroevich, K., Ross, A.J., Moore, S.J., Badano, J.L., May-Simera, H., Compton, D.S., et al. (2004). Mutations in a member of the Ras superfamily of small GTP-binding proteins causes Bardet-Biedl syndrome. Nat. Genet. 36, 989-993. Abstract Article

Fancsalszky, L., Monostori, E., Farkas, Z., Pourkarimi, E., Masoudi, N., Hargitai, B., Bosnar, M.H., Dezeljin, M., Zsakai, A., Vellai, T., et al. (2014). NDK-1, the homolog of NM23-H1/H2 regulates cell migration and apoptotic engulfment in C. elegans. PLoS One 9, e92687. Abstract Article

Feig, L.A. (2003). Ral-GTPases: approaching their 15 minutes of fame. Trends Cell Biol. 13, 419-425. Abstract Article

Feng, W., Liang, T., Yu, J., Zhou, W., Zhang, Y., Wu, Z., and Xu, T. (2012). RAB-27 and its effector RBF-1 regulate the tethering and docking steps of DCV exocytosis in C. elegans. Sci. China Life Sci. 55, 228-235. Abstract Article

Ferguson, E.L., Sternberg, P.W., and Horvitz, H.R. (1987). A genetic pathway for the specification of the vulval cell lineages of Caenorhabditis elegans. Nature 326, 259-267. Abstract Article

Fernandez, A.G., and Piano, F. (2006). MEL-28 is downstream of the Ran cycle and is required for nuclear-envelope function and chromatin maintenance. Curr. Biol. 16, 1757-1763. Abstract Article

Fernandez-Medarde, A., and Santos, E. (2011). Ras in cancer and developmental diseases. Genes Cancer 2, 344-358. Abstract Article 
Fischer, S.E., Pan, Q., Breen, P.C., Qi, Y., Shi, Z., Zhang, C., and Ruvkun, G. (2013). Multiple small RNA pathways regulate the silencing of repeated and foreign genes in C. elegans. Genes Dev. 27, 2678-2695. Abstract Article

Flex, E., Jaiswal, M., Pantaleoni, F., Martinelli, S., Strullu, M., Fansa, E.K., Caye, A., De Luca, A., Lepri, F., Dvorsky, R., et al. (2014). Activating mutations in RRAS underlie a phenotype within the RASopathy spectrum and contribute to leukaemogenesis. Hum. Mol. Genet. 23, 4315-4327. Abstract Article

Forbes, S.A., Bindal, N., Bamford, S., Cole, C., Kok, C.Y., Beare, D., Jia, M., Shepherd, R., Leung, K., Menzies, A., et al. (2011). COSMIC: mining complete cancer genomes in the Catalogue of Somatic Mutations in Cancer. Nucleic Acids Res. 39, D945-950. Abstract Article

Fotopoulos, N., Wernike, D., Chen, Y., Makil, N., Marte, A., and Piekny, A. (2013). Caenorhabditis elegans anillin (ani-1) regulates neuroblast cytokinesis and epidermal morphogenesis during embryonic development. Dev. Biol. 383, 61-74. Abstract Article

Frische, E.W., Pellis-van Berkel, W., van Haaften, G., Cuppen, E., Plasterk, R.H., Tijsterman, M., Bos, J.L., and Zwartkruis, F.J. (2007). RAP-1 and the RAL-1/exocyst pathway coordinate hypodermal cell organization in Caenorhabditis elegans. EMBO J. 26, 5083-5092. Abstract Article

Frische, E.W., and Zwartkruis, F.J. (2010). Rap1, a mercenary among the Ras-like GTPases. Dev. Biol. 340, 1-9. Abstract Article

Gabel, C.V., Antoine, F., Chuang, C.F., Samuel, A.D., and Chang, C. (2008). Distinct cellular and molecular mechanisms mediate initial axon development and adult-stage axon regeneration in C. elegans. Development 135, 1129-1136. Abstract Article

Gallegos, M.E., Balakrishnan, S., Chandramouli, P., Arora, S., Azameera, A., Babushekar, A., Bargoma, E., Bokhari, A., Chava, S.K., Das, P., et al. (2012). The C. elegans rab family: identification, classification and toolkit construction. PLoS One 7, e49387. Abstract Article

Gally, C., Wissler, F., Zahreddine, H., Quintin, S., Landmann, F., and Labouesse, M. (2009). Myosin II regulation during $C$. elegans embryonic elongation: LET-502/ROCK, MRCK-1 and PAK-1, three kinases with different roles. Development 136, 3109-3119. Abstract Article

Garcia-Mata, R., Boulter, E., and Burridge, K. (2011). The 'invisible hand': regulation of RHO GTPases by RHOGDIs. Nat. Rev. Mol. Cell Biol. 12, 493-504. Abstract Article

Gasper, R., Sot, B., and Wittinghofer, A. (2010). GTPase activity of Di-Ras proteins is stimulated by Rap1GAP proteins. Small GTPases 1, 133-141. Abstract Article

Gentry, L.R., Martin, T.D., Reiner, D.J., and Der, C.J. (2014). Ral small GTPase signaling and oncogenesis: More than just 15minutes of fame. Biochim. Biophys. Acta 1843, 2976-2988. Abstract Article

Ghosh, S., and Sternberg, P.W. (2014). Spatial and molecular cues for cell outgrowth during C. elegans uterine development. Dev. Biol. 396, 121-135. Abstract Article

Gitai, Z., Yu, T.W., Lundquist, E.A., Tessier-Lavigne, M., and Bargmann, C.I. (2003). The netrin receptor UNC-40/DCC stimulates axon attraction and outgrowth through enabled and, in parallel, Rac and UNC-115/AbLIM. Neuron 37, 53-65. Abstract Article

Giuliani, C., Troglio, F., Bai, Z., Patel, F.B., Zucconi, A., Malabarba, M.G., Disanza, A., Stradal, T.B., Cassata, G., Confalonieri, S., et al. (2009). Requirements for F-BAR proteins TOCA-1 and TOCA-2 in actin dynamics and membrane trafficking during Caenorhabditis elegans oocyte growth and embryonic epidermal morphogenesis. PLoS Genet. 5, e1000675. Abstract Article

Glodowski, D.R., Chen, C.C., Schaefer, H., Grant, B.D., and Rongo, C. (2007). RAB-10 regulates glutamate receptor recycling in a cholesterol-dependent endocytosis pathway. Mol. Biol. Cell 18, 4387-4396. Abstract Article 
Gloerich, M., ten Klooster, J.P., Vliem, M.J., Koorman, T., Zwartkruis, F.J., Clevers, H., and Bos, J.L. (2012). Rap2A links intestinal cell polarity to brush border formation. Nat. Cell Biol. 14, 793-801. Abstract Article

Goicoechea, S.M., Awadia, S., and Garcia-Mata, R. (2014). I'm coming to GEF you: Regulation of RhoGEFs during cell migration. Cell Adh. Migr. 8, 535-549. Abstract Article

Gotta, M., Abraham, M.C., and Ahringer, J. (2001). CDC-42 controls early cell polarity and spindle orientation in $C$. elegans. Curr. Biol. 11, 482-488. Abstract Article

Govindan, J.A., Cheng, H., Harris, J.E., and Greenstein, D. (2006). $\mathrm{G} \alpha_{\mathrm{o} i \mathrm{i}}$ and $\mathrm{G} \alpha_{\mathrm{s}}$ signaling function in parallel with the MSP/Eph receptor to control meiotic diapause in C. elegans. Curr. Biol. 16, 1257-1268. Abstract Article

Gracheva, E.O., Burdina, A.O., Holgado, A.M., Berthelot-Grosjean, M., Ackley, B.D., Hadwiger, G., Nonet, M.L., Weimer, R.M., and Richmond, J.E. (2006). Tomosyn inhibits synaptic vesicle priming in Caenorhabditis elegans. PLoS Biol 4, e261. Abstract Article

Gracheva, E.O., Hadwiger, G., Nonet, M.L., and Richmond, J.E. (2008). Direct interactions between C. elegans RAB-3 and Rim provide a mechanism to target vesicles to the presynaptic density. Neurosci. Lett. 444, 137-142. Abstract Article

Grant, B., and Hirsh, D. (1999). Receptor-mediated endocytosis in the Caenorhabditis elegans oocyte. Mol. Biol. Cell 10, 4311-4326. Abstract Article

Greenwald, I. LIN-12/Notch signaling in C. elegans (August 4, 2005), WormBook, ed. The C. elegans Research Community, WormBook, doi/10.1895/wormbook.1.10.1, http://www.wormbook.org. Abstract Article

Greenwald I. and Kovall R., Notch signaling: genetics and structure (January 17, 2013), WormBook, ed. The $C$. elegans Research Community, WormBook, doi/10.1895/wormbook.1.10.2, http://www.wormbook.org. Abstract Article

Grill, B., Bienvenut, W.V., Brown, H.M., Ackley, B.D., Quadroni, M., and Jin, Y. (2007). C. elegans RPM-1 regulates axon termination and synaptogenesis through the Rab GEF GLO-4 and the Rab GTPase GLO-1. Neuron 55, 587-601. Abstract Article

Groenewoud, M.J., and Zwartkruis, F.J. (2013). Rheb and Rags come together at the lysosome to activate mTORC1. Biochem. Soc. Trans. 41, 951-955. Abstract Article

Gumienny, T.L., Brugnera, E., Tosello-Trampont, A.C., Kinchen, J.M., Haney, L.B., Nishiwaki, K., Walk, S.F., Nemergut, M.E., Macara, I.G., Francis, R., et al. (2001). CED-12/ELMO, a novel member of the CrkII/Dock180/Rac pathway, is required for phagocytosis and cell migration. Cell 107, 27-41. Abstract Article

Gumienny, T.L., Lambie, E., Hartwieg, E., Horvitz, H.R., and Hengartner, M.O. (1999). Genetic control of programmed cell death in the Caenorhabditis elegans hermaphrodite germline. Development 126, 1011-1022. Abstract Article

Guo, P., Hu, T., Zhang, J., Jiang, S., and Wang, X. (2010). Sequential action of Caenorhabditis elegans Rab GTPases regulates phagolysosome formation during apoptotic cell degradation. Proc. Natl. Acad. Sci. U. S. A. 107, 18016-18021. Abstract Article

Guo, P., and Wang, X. (2010). Rab GTPases act in sequential steps to regulate phagolysosome formation. Small GTPases 1, 170-173. Abstract Article

Ha, M.K., Soo Cho, J., Baik, O.R., Lee, K.H., Koo, H.S., and Chung, K.Y. (2006). Caenorhabditis elegans as a screening tool for the endothelial cell-derived putative aging-related proteins detected by proteomic analysis. Proteomics 6, 3339-3351. Abstract Article

Hajnal, A., Whitfield, C.W., and Kim, S.K. (1997). Inhibition of Caenorhabditis elegans vulval induction by gap-1 and by let-23 receptor tyrosine kinase. Genes Dev. 11, 2715-2728. Abstract Article 
Hamad, N.M., Elconin, J.H., Karnoub, A.E., Bai, W., Rich, J.N., Abraham, R.T., Der, C.J., and Counter, C.M. (2002). Distinct requirements for Ras oncogenesis in human versus mouse cells. Genes Dev. 16, $2045-2057$. Abstract Article

Han, M., Aroian, R.V., and Sternberg, P.W. (1990). The let-60 locus controls the switch between vulval and nonvulval cell fates in Caenorhabditis elegans. Genetics 126, 899-913. Abstract Article

Han, M., Golden, A., Han, Y., and Sternberg, P.W. (1993). C. elegans lin-45 raf gene participates in let-60 ras-stimulated vulval differentiation. Nature 363, 133-140. Abstract Article

Han, M., and Sternberg, P.W. (1990). let-60, a gene that specifies cell fates during C. elegans vulval induction, encodes a ras protein. Cell 63, 921-931. Abstract Article

Han, M., and Sternberg, P.W. (1991). Analysis of dominant-negative mutations of the Caenorhabditis elegans let-60 ras gene. Genes Dev. 5, 2188-2198. Abstract Article

Hancock, J.F. (2003). Ras proteins: different signals from different locations. Nat. Rev. Mol. Cell Biol. 4, $373-384$. Abstract Article

Hannemann, M., Sasidharan, N., Hegermann, J., Kutscher, L.M., Koenig, S., and Eimer, S. (2012). TBC-8, a putative RAB-2 GAP, regulates dense core vesicle maturation in Caenorhabditis elegans. PLoS Genet. 8, e1002722. Abstract Article

Hara, K., Maruki, Y., Long, X., Yoshino, K., Oshiro, N., Hidayat, S., Tokunaga, C., Avruch, J., and Yonezawa, K. (2002). Raptor, a binding partner of target of rapamycin (TOR), mediates TOR action. Cell 110, 177-189. Abstract Article

Hara, M., and Han, M. (1995). Ras farnesyltransferase inhibitors suppress the phenotype resulting from an activated ras mutation in Caenorhabditis elegans. Proc. Natl. Acad. Sci. U. S. A. 92, 3333-3337. Abstract Article

Haynes, C.M., Petrova, K., Benedetti, C., Yang, Y., and Ron, D. (2007). ClpP mediates activation of a mitochondrial unfolded protein response in C. elegans. Dev. Cell 13, 467-480. Abstract Article

He, B., Yu, X., Margolis, M., Liu, X., Leng, X., Etzion, Y., Zheng, F., Lu, N., Quiocho, F.A., Danino, D., et al. (2010). Live-cell imaging in Caenorhabditis elegans reveals the distinct roles of dynamin self-assembly and guanosine triphosphate hydrolysis in the removal of apoptotic cells. Mol. Biol. Cell 21, 610-629. Abstract Article

Hermann, G.J., Schroeder, L.K., Hieb, C.A., Kershner, A.M., Rabbitts, B.M., Fonarev, P., Grant, B.D., and Priess, J.R. (2005). Genetic analysis of lysosomal trafficking in Caenorhabditis elegans. Mol. Biol. Cell 16, $3273-3288$. Abstract Article

Hikita, T., Qadota, H., Tsuboi, D., Taya, S., Moerman, D.G., and Kaibuchi, K. (2005). Identification of a novel Cdc42 GEF that is localized to the PAT-3-mediated adhesive structure. Biochem. Biophys. Res. Commun. 335, 139-145. Abstract Article

Hiley, E., McMullan, R., and Nurrish, S.J. (2006). The G $112-$ RGS RhoGEF-RhoA signalling pathway regulates neurotransmitter release in C. elegans. EMBO J. 25, 5884-5895. Abstract Article

Hirotsu, T., Saeki, S., Yamamoto, M., and Iino, Y. (2000). The Ras-MAPK pathway is important for olfaction in Caenorhabditis elegans. Nature 404, 289-293. Abstract Article

Hodis, E., Watson, I.R., Kryukov, G.V., Arold, S.T., Imielinski, M., Theurillat, J.P., Nickerson, E., Auclair, D., Li, L., Place, C., et al. (2012). A landscape of driver mutations in melanoma. Cell 150, 251-263. Abstract Article

Holbrook, S.R., and Kim, S.H. (1989). Molecular model of the G protein $\alpha$ subunit based on the crystal structure of the HRAS protein. Proc. Natl. Acad. Sci. U. S. A. 86, 1751-1755. Abstract Article

Honjoh, S., and Nishida, E. (2011). Two sides of lifespan regulating genes: pro-longevity or anti-longevity? J. Biochem. 149, 381-388. Abstract Article

Honjoh, S., Yamamoto, T., Uno, M., and Nishida, E. (2009). Signalling through RHEB-1 mediates intermittent fasting-induced longevity in C. elegans. Nature 457, 726-730. Abstract Article 
Hsieh, H.H., Hsu, T.Y., Jiang, H.S., and Wu, Y.C. (2012). Integrin $\alpha$ PAT-2/CDC-42 signaling is required for muscle-mediated clearance of apoptotic cells in Caenorhabditis elegans. PLoS Genet. 8, e1002663. Abstract Article

Hsu, Y.C., Chern, J.J., Cai, Y., Liu, M., and Choi, K.W. (2007). Drosophila TCTP is essential for growth and proliferation through regulation of dRheb GTPase. Nature 445, 785-788. Abstract Article

Hu, P.J. Dauer (August 08, 2007), WormBook, ed. The C. elegans Research Community, WormBook, doi/10.1895/wormbook.1.144.1, http://www.wormbook.org. Abstract Article

Hu, S., Pawson, T., and Steven, R.M. (2011). UNC-73/trio RhoGEF-2 activity modulates Caenorhabditis elegans motility through changes in neurotransmitter signaling upstream of the GSA-1/Galphas pathway. Genetics 189, 137-151. Abstract Article

Hung, T.J., and Kemphues, K.J. (1999). PAR-6 is a conserved PDZ domain-containing protein that colocalizes with PAR-3 in Caenorhabditis elegans embryos. Development 126, 127-135. Abstract Article

Hurwitz, M.E., Vanderzalm, P.J., Bloom, L., Goldman, J., Garriga, G., and Horvitz, H.R. (2009). Abl kinase inhibits the engulfment of apoptotic cells in Caenorhabditis elegans. PLoS Biol. 7, e99. Abstract Article

Hyenne, V., Tremblay-Boudreault, T., Velmurugan, R., Grant, B.D., Loerke, D., and Labbe, J.C. (2012). RAB-5 controls the cortical organization and dynamics of PAR proteins to maintain $C$. elegans early embryonic polarity. PLoS One 7, e35286. Abstract Article

Ikegami, R., Simokat, K., Zheng, H., Brown, L., Garriga, G., Hardin, J., and Culotti, J. (2012). Semaphorin and Eph receptor signaling guide a series of cell movements for ventral enclosure in C. elegans. Curr. Biol. $22,1-11$. Abstract Article

Ikegami, R., Zheng, H., Ong, S.H., and Culotti, J. (2004). Integration of semaphorin-2A/MAB-20, ephrin-4, and UNC-129 TGF- $\beta$ signaling pathways regulates sorting of distinct sensory rays in C. elegans. Dev. Cell 6, $383-395$. Abstract Article

Inoki, K., Li, Y., Xu, T., and Guan, K.L. (2003). Rheb GTPase is a direct target of TSC2 GAP activity and regulates mTOR signaling. Genes Dev. 17, 1829-1834. Abstract Article Article

Ivanov, I.P., Firth, A.E., Michel, A.M., Atkins, J.F., and Baranov, P.V. (2011). Identification of evolutionarily conserved non-AUG-initiated N-terminal extensions in human coding sequences. Nucleic Acids Res. 39, 4220-4234. Abstract Article

Iwasaki, K., Staunton, J., Saifee, O., Nonet, M., and Thomas, J.H. (1997). aex-3 encodes a novel regulator of presynaptic activity in C. elegans. Neuron 18,613-622. Abstract Article

Iwasaki, K., and Toyonaga, R. (2000). The Rab3 GDP/GTP exchange factor homolog AEX-3 has a dual function in synaptic transmission. EMBO J. 19, 4806-4816. Abstract Article

Jackson, J.H., Cochrane, C.G., Bourne, J.R., Solski, P.A., Buss, J.E., and Der, C.J. (1990). Farnesol modification of Kirsten-ras exon 4B protein is essential for transformation. Proc. Natl. Acad. Sci. U. S. A. 87, 3042-3046. Abstract Article

Jantsch-Plunger, V., Gonczy, P., Romano, A., Schnabel, H., Hamill, D., Schnabel, R., Hyman, A.A., and Glotzer, M. (2000). CYK-4: A Rho family gtpase activating protein (GAP) required for central spindle formation and cytokinesis. J. Cell Biol. 149, 1391-1404. Abstract Article

Jenkins, N., Saam, J.R., and Mango, S.E. (2006). CYK-4/GAP provides a localized cue to initiate anteroposterior polarity upon fertilization. Science 313, 1298-1301. Abstract Article

Jenna, S., Caruso, M.E., Emadali, A., Nguyen, D.T., Dominguez, M., Li, S., Roy, R., Reboul, J., Vidal, M., Tzimas, G.N., et al. (2005). Regulation of membrane trafficking by a novel Cdc42-related protein in Caenorhabditis elegans epithelial cells. Mol. Biol. Cell 16, 1629-1639. Abstract Article 
Jenzer, C., Manil-Segalen, M., Lefebvre, C., Largeau, C., Glatigny, A., and Legouis, R. (2014). Human GABARAP can restore autophagosome biogenesis in a C. elegans lgg-1 mutant. Autophagy 10, 1868-1872. Abstract Article

Jewell, J.L., Russell, R.C., and Guan, K.L. (2013). Amino acid signalling upstream of mTOR. Nat. Rev. Mol. Cell Biol. 14, 133-139. Abstract Article

Jia, K., Chen, D., and Riddle, D.L. (2004). The TOR pathway interacts with the insulin signaling pathway to regulate $C$. elegans larval development, metabolism and life span. Development 131, 3897-3906. Abstract Article

Jiang, L.I., and Sternberg, P.W. (1998). Interactions of EGF, Wnt and HOM-C genes specify the P12 neuroectoblast fate in C. elegans. Development 125, 2337-2347. Abstract Article

Jiu, Y., Jin, C., Liu, Y., Holmberg, C.I., and Jantti, J. (2012). Exocyst subunits Exo70 and Exo84 cooperate with small GTPases to regulate behavior and endocytic trafficking in C. elegans. PLoS One 7, e32077. Abstract Article

Johnson, J.R., Kashyap, S., Rankin, K., and Barclay, J.W. (2013). rab-3 and unc-18 interactions in alcohol sensitivity are distinct from synaptic transmission. PLoS One 8, e81117. Abstract Article

Jones, K.T., Greer, E.R., Pearce, D., and Ashrafi, K. (2009). Rictor/TORC2 regulates Caenorhabditis elegans fat storage, body size, and development through sgk-1. PLoS Biol. 7, e60. Abstract Article

Kaech, S.M., Whitfield, C.W., and Kim, S.K. (1998). The LIN-2/LIN-7/LIN-10 complex mediates basolateral membrane localization of the $C$. elegans EGF receptor LET-23 in vulval epithelial cells. Cell 94, 761-771. Abstract Article

Kalab, P., Pralle, A., Isacoff, E.Y., Heald, R., and Weis, K. (2006). Analysis of a RanGTP-regulated gradient in mitotic somatic cells. Nature 440, 697-701. Abstract Article

Kalab, P., Weis, K., and Heald, R. (2002). Visualization of a Ran-GTP gradient in interphase and mitotic Xenopus egg extracts. Science 295, 2452-2456. Abstract Article

Kalis, A.K., Kroetz, M.B., Larson, K.M., and Zarkower, D. (2010). Functional genomic identification of genes required for male gonadal differentiation in Caenorhabditis elegans. Genetics 185, 523-535. Abstract Article

Kapfhamer, D., Bettinger, J.C., Davies, A.G., Eastman, C.L., Smail, E.A., Heberlein, U., and McIntire, S.L. (2008). Loss of RAB-3/A in Caenorhabditis elegans and the mouse affects behavioral response to ethanol. Genes Brain Behav. 7, 669-676. Abstract Article

Kaplan, O.I., Doroquez, D.B., Cevik, S., Bowie, R.V., Clarke, L., Sanders, A.A., Kida, K., Rappoport, J.Z., Sengupta, P., and Blacque, O.E. (2012). Endocytosis genes facilitate protein and membrane transport in C. elegans sensory cilia. Curr. Biol. 22, 451-460. Abstract Article

Kaplan, O.I., Molla-Herman, A., Cevik, S., Ghossoub, R., Kida, K., Kimura, Y., Jenkins, P., Martens, J.R., Setou, M., Benmerah, A., et al. (2010). The AP-1 clathrin adaptor facilitates cilium formation and functions with RAB-8 in C. elegans ciliary membrane transport. J. Cell Sci. 123, 3966-3977. Abstract Article

Kariya, K., Bui, Y.K., Gao, X., Sternberg, P.W., and Kataoka, T. (2004). Phospholipase C-E regulates ovulation in Caenorhabditis elegans. Dev. Biol. 274, 201-210. Abstract Article

Kashatus, D.F., Lim, K.H., Brady, D.C., Pershing, N.L., Cox, A.D., and Counter, C.M. (2011). RALA and RALBP1 regulate mitochondrial fission at mitosis. Nat. Cell Biol. 13, 1108-1115. Abstract Article

Katsanis, N. (2004). The oligogenic properties of Bardet-Biedl syndrome. Hum. Mol. Genet. 13 Spec No 1, R65-71. Abstract Article

Katz, W.S., Hill, R.J., Clandinin, T.R., and Sternberg, P.W. (1995). Different levels of the C. elegans growth factor LIN-3 promote distinct vulval precursor fates. Cell 82, 297-307. Abstract Article

Katz, W.S., Lesa, G.M., Yannoukakos, D., Clandinin, T.R., Schlessinger, J., and Sternberg, P.W. (1996). A point mutation in the extracellular domain activates LET-23, the Caenorhabditis elegans epidermal growth factor receptor homolog. Mol. Cell. Biol. 16, 529-537. Abstract Article 
Kay, A.J., and Hunter, C.P. (2001). CDC-42 regulates PAR protein localization and function to control cellular and embryonic polarity in C. elegans. Curr. Biol. 11, 474-481. Abstract Article

Kemphues, K.J., Priess, J.R., Morton, D.G., and Cheng, N.S. (1988). Identification of genes required for cytoplasmic localization in early C. elegans embryos. Cell 52, 311-320. Abstract Article

Kenyon, C. (1995). A perfect vulva every time: gradients and signaling cascades in C. elegans. Cell 82, 171-174. Abstract Article

Kidd, A.R., 3rd, Muniz-Medina, V., Der, C.J., Cox, A.D., and Reiner, D.J. (2015). The C. elegans Chp/Wrch ortholog CHW-1 contributes to LIN-18/Ryk and LIN-17/Frizzled signaling in cell polarity. PLoS One 10, e0133226. Abstract Article

Kikuchi, T., Shibata, Y., Kim, H.S., Kubota, Y., Yoshina, S., Mitani, S., and Nishiwaki, K. (2015). The BED finger domain protein MIG-39 halts migration of distal tip cells in Caenorhabditis elegans. Dev. Biol. 397, 151-161. Abstract Article

Kim, Y., and Sun, H. (2007). Functional genomic approach to identify novel genes involved in the regulation of oxidative stress resistance and animal lifespan. Aging Cell 6, 489-503. Abstract Article

Kimura, K., and Kimura, A. (2012). Rab6 is required for the exocytosis of cortical granules and the recruitment of separase to the granules during the oocyte-to-embryo transition in Caenorhabditis elegans. J. Cell Sci. 125, 5897-5905. Abstract Article

Kinchen, J.M., Cabello, J., Klingele, D., Wong, K., Feichtinger, R., Schnabel, H., Schnabel, R., and Hengartner, M.O. (2005). Two pathways converge at CED-10 to mediate actin rearrangement and corpse removal in C. elegans. Nature 434, 93-99. Abstract Article

Kinchen, J.M., Doukoumetzidis, K., Almendinger, J., Stergiou, L., Tosello-Trampont, A., Sifri, C.D., Hengartner, M.O., and Ravichandran, K.S. (2008). A pathway for phagosome maturation during engulfment of apoptotic cells. Nat. Cell Biol. 10, 556-566. Abstract Article

Kinchen, J.M., and Ravichandran, K.S. (2010). Identification of two evolutionarily conserved genes regulating processing of engulfed apoptotic cells. Nature 464, 778-782. Abstract Article

Kishore, R.S., and Sundaram, M.V. (2002). ced-10 Rac and mig-2 function redundantly and act with unc-73 Trio to control the orientation of vulval cell divisions and migrations in Caenorhabditis elegans. Dev. Biol. 241, 339-348. Abstract Article

Klassen, M.P., Wu, Y.E., Maeder, C.I., Nakae, I., Cueva, J.G., Lehrman, E.K., Tada, M., Gengyo-Ando, K., Wang, G.J., Goodman, M., et al. (2010). An Arf-like small G protein, ARL-8, promotes the axonal transport of presynaptic cargoes by suppressing vesicle aggregation. Neuron 66,710-723. Abstract Article

Kniazeva, M., Crawford, Q.T., Seiber, M., Wang, C.Y., and Han, M. (2004). Monomethyl branched-chain fatty acids play an essential role in Caenorhabditis elegans development. PLoS Biol. 2, E257. Abstract Article

Knobel, K.M., Jorgensen, E.M., and Bastiani, M.J. (1999). Growth cones stall and collapse during axon outgrowth in Caenorhabditis elegans. Development 126, 4489-4498. Abstract Article

Koga, M., and Ohshima, Y. (1995). Mosaic analysis of the let-23 gene function in vulval induction of Caenorhabditis elegans. Development 121, 2655-2666. Abstract Article

Kooistra, M.R., Dube, N., and Bos, J.L. (2007). Rap1: a key regulator in cell-cell junction formation. J. Cell Sci. 120, 17-22. Abstract Article

Koushika, S.P., Richmond, J.E., Hadwiger, G., Weimer, R.M., Jorgensen, E.M., and Nonet, M.L. (2001). A post-docking role for active zone protein Rim. Nat. Neurosci. 4, 997-1005. Abstract Article

Krauthammer, M., Kong, Y., Ha, B.H., Evans, P., Bacchiocchi, A., McCusker, J.P., Cheng, E., Davis, M.J., Goh, G., Choi, M., et al. (2012). Exome sequencing identifies recurrent somatic RAC1 mutations in melanoma. Nat. Genet. 44, 1006-1014. Abstract Article 
Kumfer, K.T., Cook, S.J., Squirrell, J.M., Eliceiri, K.W., Peel, N., O'Connell, K.F., and White, J.G. (2010). CGEF-1 and CHIN-1 regulate CDC-42 activity during asymmetric division in the Caenorhabditis elegans embryo. Mol. Biol. Cell 21, 266-277. Abstract Article

Kuwahara, T., Koyama, A., Koyama, S., Yoshina, S., Ren, C.H., Kato, T., Mitani, S., and Iwatsubo, T. (2008). A systematic RNAi screen reveals involvement of endocytic pathway in neuronal dysfunction in $\alpha$-synuclein transgenic C. elegans. Hum. Mol. Genet. 17, 2997-3009. Abstract Article

Kyriakis, J.M., App, H., Zhang, X.F., Banerjee, P., Brautigan, D.L., Rapp, U.R., and Avruch, J. (1992). Raf-1 activates MAP kinase-kinase. Nature 358, 417-421. Abstract Article

Lawson, C.D., and Burridge, K. (2014). The on-off relationship of Rho and Rac during integrin-mediated adhesion and cell migration. Small GTPases 5, e27958. Abstract Article

Lee, J., Jongeward, G.D., and Sternberg, P.W. (1994). unc-101, a gene required for many aspects of Caenorhabditis elegans development and behavior, encodes a clathrin-associated protein. Genes Dev. 8, 60-73. Abstract Article

Lerner, E.C., Zhang, T.T., Knowles, D.B., Qian, Y., Hamilton, A.D., and Sebti, S.M. (1997). Inhibition of the prenylation of K-Ras, but not H- or N-Ras, is highly resistant to CAAX peptidomimetics and requires both a farnesyltransferase and a geranylgeranyltransferase I inhibitor in human tumor cell lines. Oncogene 15, 1283-1288. Abstract Article

Leto, D., Uhm, M., Williams, A., Chen, X.W., and Saltiel, A.R. (2013). Negative regulation of the RalGAP complex by 14-3-3. J. Biol. Chem. 288, 9272-9283. Abstract Article

Levitan, D.J., Boyd, L., Mello, C.C., Kemphues, K.J., and Stinchcomb, D.T. (1994). par-2, a gene required for blastomere asymmetry in Caenorhabditis elegans, encodes zinc-finger and ATP-binding motifs. Proc. Natl. Acad. Sci. U. S. A. 91, 6108-6112. Abstract Article

Li, J.B., Gerdes, J.M., Haycraft, C.J., Fan, Y., Teslovich, T.M., May-Simera, H., Li, H., Blacque, O.E., Li, L., Leitch, C.C., et al. (2004). Comparative genomics identifies a flagellar and basal body proteome that includes the BBS5 human disease gene. Cell 117, 541-552. Abstract Article

Li, W., Zou, W., Yang, Y., Chai, Y., Chen, B., Cheng, S., Tian, D., Wang, X., Vale, R.D., and Ou, G. (2012). Autophagy genes function sequentially to promote apoptotic cell corpse degradation in the engulfing cell. J. Cell Biol. 197, 27-35. Abstract Article

Li, W., Zou, W., Zhao, D., Yan, J., Zhu, Z., Lu, J., and Wang, X. (2009). C. elegans Rab GTPase activating protein TBC-2 promotes cell corpse degradation by regulating the small GTPase RAB-5. Development 136, 2445-2455. Abstract Article

Li, Y., Kelly, W.G., Logsdon, J.M., Jr., Schurko, A.M., Harfe, B.D., Hill-Harfe, K.L., and Kahn, R.A. (2004). Functional genomic analysis of the ADP-ribosylation factor family of GTPases: phylogeny among diverse eukaryotes and function in C. elegans. FASEB J. 18, 1834-1850. Abstract Article

Li, Y., Wei, Q., Zhang, Y., Ling, K., and Hu, J. (2010). The small GTPases ARL-13 and ARL-3 coordinate intraflagellar transport and ciliogenesis. J. Cell Biol. 189, 1039-1051. Abstract Article

Lin, L., Tran, T., Hu, S., Cramer, T., Komuniecki, R., and Steven, R.M. (2012). RHGF-2 is an essential Rho-1 specific RhoGEF that binds to the multi-PDZ domain scaffold protein MPZ-1 in Caenorhabditis elegans. PLoS One 7, e31499. Abstract Article

Liu, O., and Grant, B.D. (2015). Basolateral endocytic recycling requires RAB-10 and AMPH-1 mediated recruitment of RAB-5 GAP TBC-2 toendosomes. PLoS Genet. 11, e1005514. Abstract Article

Locke, C.J., Kautu, B.B., Berry, K.P., Lee, S.K., Caldwell, K.A., and Caldwell, G.A. (2009). Pharmacogenetic analysis reveals a post-developmental role for Rac GTPases in Caenorhabditis elegans GABAergic neurotransmission. Genetics 183, 1357-1372. Abstract Article 
Long, X., Spycher, C., Han, Z.S., Rose, A.M., Muller, F., and Avruch, J. (2002). TOR deficiency in C. elegans causes developmental arrest and intestinal atrophy by inhibition of mRNA translation. Curr. Biol. 12, $1448-1461$. Abstract Article

Los, F.C., Kao, C.Y., Smitham, J., McDonald, K.L., Ha, C., Peixoto, C.A., and Aroian, R.V. (2011). RAB-5- and RAB-11-dependent vesicle-trafficking pathways are required for plasma membrane repair after attack by bacterial pore-forming toxin. Cell Host Microbe 9, 147-157. Abstract

Lowenstein, E.J., Daly, R.J., Batzer, A.G., Li, W., Margolis, B., Lammers, R., Ullrich, A., Skolnik, E.Y., Bar-Sagi, D., and Schlessinger, J. (1992). The SH2 and SH3 domain-containing protein GRB2 links receptor tyrosine kinases to ras signaling. Cell 70, 431-442. Abstract Article

Lu, M., Kinchen, J.M., Rossman, K.L., Grimsley, C., deBakker, C., Brugnera, E., Tosello-Trampont, A.C., Haney, L.B., Klingele, D., Sondek, J., et al. (2004). PH domain of ELMO functions in trans to regulate Rac activation via Dock180. Nat. Struct. Mol. Biol. 11, 756-762. Abstract Article

Lu, N., Shen, Q., Mahoney, T.R., Liu, X., and Zhou, Z. (2011). Three sorting nexins drive the degradation of apoptotic cells in response to PtdIns(3)P signaling. Mol. Biol. Cell 22, 354-374. Abstract Article

Lu, N., Shen, Q., Mahoney, T.R., Neukomm, L.J., Wang, Y., and Zhou, Z. (2012). Two PI 3-kinases and one PI 3-phosphatase together establish the cyclic waves of phagosomal PtdIns(3)P critical for the degradation of apoptotic cells. PLoS Biol. 10, e1001245. Abstract Article

Lu, Q., Zhang, Y., Hu, T., Guo, P., Li, W., and Wang, X. (2008). C. elegans Rab GTPase 2 is required for the degradation of apoptotic cells. Development 135, 1069-1080. Abstract Article

Lucanic, M., and Cheng, H.J. (2008). A RAC/CDC-42-independent GIT/PIX/PAK signaling pathway mediates cell migration in C. elegans. PLoS Genet. 4, e1000269. Abstract Article

Lucanic, M., Kiley, M., Ashcroft, N., L'Etoile, N., and Cheng, H.J. (2006). The Caenorhabditis elegans P21-activated kinases are differentially required for UNC-6/netrin-mediated commissural motor axon guidance. Development 133, 4549-4559. Abstract Article

Lundquist, E.A., Reddien, P.W., Hartwieg, E., Horvitz, H.R., and Bargmann, C.I. (2001). Three C. elegans Rac proteins and several alternative Rac regulators control axon guidance, cell migration and apoptotic cell phagocytosis. Development 128, 4475-4488. Abstract Article

Luo, L., Hannemann, M., Koenig, S., Hegermann, J., Ailion, M., Cho, M.K., Sasidharan, N., Zweckstetter, M., Rensing, S.A., and Eimer, S. (2011). The Caenorhabditis elegans GARP complex contains the conserved Vps51 subunit and is required to maintain lysosomal morphology. Mol. Biol. Cell 22, 2564-2578. Abstract Article

Maduzia, L.L., Moreau, A., Poullet, N., Chaffre, S., and Zhang, Y. (2010). The role of eIF1 in translation initiation codon selection in Caenorhabditis elegans. Genetics 186, 1187-1196. Abstract Article

Mahoney, T.R., Liu, Q., Itoh, T., Luo, S., Hadwiger, G., Vincent, R., Wang, Z.W., Fukuda, M., and Nonet, M.L. (2006). Regulation of synaptic transmission by RAB-3 and RAB-27 in Caenorhabditis elegans. Mol. Biol. Cell 17, 2617-2625. Abstract Article

Mangahas, P.M., Yu, X., Miller, K.G., and Zhou, Z. (2008). The small GTPase Rab2 functions in the removal of apoptotic cells in Caenorhabditis elegans. J. Cell Biol. 180, 357-373. Abstract Article

Maniar, T.A., Kaplan, M., Wang, G.J., Shen, K., Wei, L., Shaw, J.E., Koushika, S.P., and Bargmann, C.I. (2012). UNC-33 (CRMP) and ankyrin organize microtubules and localize kinesin to polarize axon-dendrite sorting. Nat. Neurosci. 15, 48-56. Abstract Article

Manil-Segalen, M., Lefebvre, C., Jenzer, C., Trichet, M., Boulogne, C., Satiat-Jeunemaitre, B., and Legouis, R. (2014). The $C$. elegans LC3 acts downstream of GABARAP to degrade autophagosomes by interacting with the HOPS subunit VPS39. Dev. Cell 28, 43-55. Abstract Article 
Martin, E., Harel, S., Nkengfac, B., Hamiche, K., Neault, M., and Jenna, S. (2014). pix-1 controls early elongation in parallel with mel-11 and let-502 in Caenorhabditis elegans. PLoS One 9, e94684. Abstract Article

Martin, T.D., Chen, X.W., Kaplan, R.E., Saltiel, A.R., Walker, C.L., Reiner, D.J., and Der, C.J. (2014). Ral and Rheb GTPase activating proteins integrate mTOR and GTPase signaling in aging, autophagy, and tumor cell invasion. Mol. Cell 53, 209-220. Abstract Article

Mavromatakis, Y.E., and Tomlinson, A. (2012). The role of the small GTPase Rap in Drosophila R7 photoreceptor specification. Proc. Natl. Acad. Sci. U. S. A. 109, 3844-3849. Abstract Article

Mazhab-Jafari, M.T., Marshall, C.B., Ho, J., Ishiyama, N., Stambolic, V., and Ikura, M. (2014). Structure-guided mutation of the conserved G3-box glycine in Rheb generates a constitutively activated regulator of mammalian target of rapamycin (mTOR). J. Biol. Chem. 289, 12195-12201. Abstract Article

Mazure, N.M., Brahimi-Horn, M.C., Berta, M.A., Benizri, E., Bilton, R.L., Dayan, F., Ginouves, A., Berra, E., and Pouyssegur, J. (2004). HIF-1: master and commander of the hypoxic world. A pharmacological approach to its regulation by siRNAs. Biochem. Pharmacol. 68, 971-980. Abstract Article

McMullan, R., Anderson, A., and Nurrish, S. (2012). Behavioral and immune responses to infection require GaqRhoA signaling in C. elegans. PLoS Pathog. 8, e1002530. Abstract Article

McMullan, R., Hiley, E., Morrison, P., and Nurrish, S.J. (2006). Rho is a presynaptic activator of neurotransmitter release at pre-existing synapses in C. elegans. Genes Dev. 20,65-76. Abstract Article

McMullan, R., and Nurrish, S.J. (2011). The RHO-1 RhoGTPase modulates fertility and multiple behaviors in adult C. elegans. PLoS One 6, e17265. Abstract Article

Meléndez, A. and Levine, B. Autophagy in C. elegans (August 24, 2009), WormBook, ed. The C. elegans Research Community, WormBook, doi/10.1895/wormbook.1.147.1, http://www.wormbook.org. Abstract Article

Mirey, G., Balakireva, M., L'Hoste, S., Rosse, C., Voegeling, S., and Camonis, J. (2003). A Ral guanine exchange factor-Ral pathway is conserved in Drosophila melanogaster and sheds new light on the connectivity of the Ral, Ras, and Rap pathways. Mol. Cell. Biol. 23, 1112-1124. Abstract Article

Mishra, S., Smolik, S.M., Forte, M.A., and Stork, P.J. (2005). Ras-independent activation of ERK signaling via the Torso receptor tyrosine kinase is mediated by Rap1. Curr. Biol. 15, 366-370. Abstract Article

Mitin, N., Betts, L., Yohe, M.E., Der, C.J., Sondek, J., and Rossman, K.L. (2007). Release of autoinhibition of ASEF by APC leads to CDC42 activation and tumor suppression. Nat. Struct. Mol. Biol. 14, 814-823. Abstract Article

Modzelewska, K., Elgort, M.G., Huang, J., Jongeward, G., Lauritzen, A., Yoon, C.H., Sternberg, P.W., and Moghal, N. (2007). An activating mutation in sos- 1 identifies its Dbl domain as a critical inhibitor of the epidermal growth factor receptor pathway during Caenorhabditis elegans vulval development. Mol. Cell. Biol. 27, 3695-3707. Abstract Article

Moodie, S.A., and Wolfman, A. (1994). The 3Rs of life: Ras, Raf and growth regulation. Trends Genet. 10, 44-48. Abstract Article

Morck, C., Olsen, L., Kurth, C., Persson, A., Storm, N.J., Svensson, E., Jansson, J.O., Hellqvist, M., Enejder, A., Faergeman, N.J., et al. (2009). Statins inhibit protein lipidation and induce the unfolded protein response in the non-sterol producing nematode Caenorhabditis elegans. Proc. Natl. Acad. Sci. U. S. A. 106, 18285-18290. Abstract Article

Morita, K., Hirono, K., and Han, M. (2005). The Caenorhabditis elegans ect-2 RhoGEF gene regulates cytokinesis and migration of epidermal P cells. EMBO Rep. 6, 1163-1168. Abstract Article

Motegi, F., and Sugimoto, A. (2006). Sequential functioning of the ECT-2 RhoGEF, RHO-1 and CDC-42 establishes cell polarity in Caenorhabditis elegans embryos. Nat. Cell Biol. 8, 978-985. Abstract Article 
Mukhopadhyay, S., Lu, Y., Shaham, S., and Sengupta, P. (2008). Sensory signaling-dependent remodeling of olfactory cilia architecture in C. elegans. Dev. Cell 14, 762-774. Abstract Article

Nakae, I., Fujino, T., Kobayashi, T., Sasaki, A., Kikko, Y., Fukuyama, M., Gengyo-Ando, K., Mitani, S., Kontani, K., and Katada, T. (2010). The Arf-like GTPase Arl8 mediates delivery of endocytosed macromolecules to lysosomes in Caenorhabditis elegans. Mol. Biol. Cell 21, 2434-2442. Abstract Article

Nakagawa, A., Sullivan, K.D., and Xue, D. (2014). Caspase-activated phosphoinositide binding by CNT-1 promotes apoptosis by inhibiting the AKT pathway. Nat. Struct. Mol. Biol. 21, 1082-1090. Abstract Article

Nakayama, Y., Shivas, J.M., Poole, D.S., Squirrell, J.M., Kulkoski, J.M., Schleede, J.B., and Skop, A.R. (2009). Dynamin participates in the maintenance of anterior polarity in the Caenorhabditis elegans embryo. Dev. Cell 16, 889-900. Abstract Article

Nakdimon, I., Walser, M., Frohli, E., and Hajnal, A. (2012). PTEN negatively regulates MAPK signaling during Caenorhabditis elegans vulval development. PLoS Genet. 8, e1002881. Abstract Article

Neel, N.F., Martin, T.D., Stratford, J.K., Zand, T.P., Reiner, D.J., and Der, C.J. (2011). The RalGEF-Ral effector signaling network: the road less traveled for anti-Ras drug discovery. Genes Cancer 2, 275-287. Abstract Article

Nelson, M.D., Zhou, E., Kiontke, K., Fradin, H., Maldonado, G., Martin, D., Shah, K., and Fitch, D.H. (2011). A bow-tie genetic architecture for morphogenesis suggested by a genome-wide RNAi screen in Caenorhabditis elegans. PLoS Genet. 7, e1002010. Abstract Article

Neukomm, L.J., Frei, A.P., Cabello, J., Kinchen, J.M., Zaidel-Bar, R., Ma, Z., Haney, L.B., Hardin, J., Ravichandran, K.S., Moreno, S., et al. (2011). Loss of the RhoGAP SRGP-1 promotes the clearance of dead and injured cells in Caenorhabditis elegans. Nat. Cell Biol. 13, 79-86. Abstract Article

Neukomm, L.J., Nicot, A.S., Kinchen, J.M., Almendinger, J., Pinto, S.M., Zeng, S., Doukoumetzidis, K., Tronchere, H., Payrastre, B., Laporte, J.F., et al. (2011). The phosphoinositide phosphatase MTM-1 regulates apoptotic cell corpse clearance through CED-5-CED-12 in C. elegans. Development 138, 2003-2014. Abstract Article

Neukomm, L.J., Zeng, S., Frei, A.P., Huegli, P.A., and Hengartner, M.O. (2014). Small GTPase CDC-42 promotes apoptotic cell corpse clearance in response to PAT-2 and CED-1 in C. elegans. Cell Death Differ. 21, 845-853. Abstract Article

Nieto, C., Almendinger, J., Gysi, S., Gomez-Orte, E., Kaech, A., Hengartner, M.O., Schnabel, R., Moreno, S., and Cabello, J. (2010). ccz-1 mediates the digestion of apoptotic corpses in C. elegans. J. Cell Sci. 123, 2001-2007. Abstract Article

Nilsson, L., Conradt, B., Ruaud, A.F., Chen, C.C., Hatzold, J., Bessereau, J.L., Grant, B.D., and Tuck, S. (2008). Caenorhabditis elegans num-1 negatively regulates endocytic recycling. Genetics 179, 375-387. Abstract Article

Nobes, C.D., and Hall, A. (1995). Rho, Rac, and Cdc42 GTPases regulate the assembly of multimolecular focal complexes associated with actin stress fibers, lamellipodia, and filopodia. Cell 81, 53-62. Abstract Article

Nonet, M.L., Staunton, J.E., Kilgard, M.P., Fergestad, T., Hartwieg, E., Horvitz, H.R., Jorgensen, E.M., and Meyer, B.J. (1997). Caenorhabditis elegans rab-3 mutant synapses exhibit impaired function and are partially depleted of vesicles. J. Neurosci. 17, 8061-8073. Abstract Article

Norris, A.D., Dyer, J.O., and Lundquist, E.A. (2009). The Arp2/3 complex, UNC-115/abLIM, and UNC-34/Enabled regulate axon guidance and growth cone filopodia formation in Caenorhabditis elegans. Neural Dev. 4, 38. Abstract Article

Norris, A.D., and Lundquist, E.A. (2011). UNC-6/netrin and its receptors UNC-5 and UNC-40/DCC modulate growth cone protrusion in vivo in C. elegans. Development 138, 4433-4442. Abstract Article

Norris, A.D., Sundararajan, L., Morgan, D.E., Roberts, Z.J., and Lundquist, E.A. (2014). The UNC-6/Netrin receptors UNC-40/DCC and UNC-5 inhibit growth cone filopodial protrusion via UNC-73/Trio, Rac-like GTPases and UNC-33/CRMP. Development 141, 4395-4405. Abstract Article 
Novick, P., and Guo, W. (2002). Ras family therapy: Rab, Rho and Ral talk to the exocyst. Trends Cell Biol. 12, 247-249. Abstract Article

Ostrem, J.M., Peters, U., Sos, M.L., Wells, J.A., and Shokat, K.M. (2013). K-Ras(G12C) inhibitors allosterically control GTP affinity and effector interactions. Nature 503, 548-551. Abstract Article

Ou, G., and Vale, R.D. (2009). Molecular signatures of cell migration in C. elegans Q neuroblasts. J. Cell Biol. 185, 77-85. Abstract Article

Parry, D.H., Xu, J., and Ruvkun, G. (2007). A whole-genome RNAi Screen for C. elegans miRNA pathway genes. Curr. Biol. 17, 2013-2022. Abstract Article

Parry, J.M., and Sundaram, M.V. (2014). A non-cell-autonomous role for Ras signaling in C. elegans neuroblast delamination. Development 141, 4279-4284. Abstract Article

Patel, F.B., Bernadskaya, Y.Y., Chen, E., Jobanputra, A., Pooladi, Z., Freeman, K.L., Gally, C., Mohler, W.A., and Soto, M.C. (2008). The WAVE/SCAR complex promotes polarized cell movements and actin enrichment in epithelia during C. elegans embryogenesis. Dev. Biol. 324, 297-309. Abstract Article

Pawson, T. (1992). Cell signalling. Conviction by genetics. Nature 356, 285-286. Abstract Article

Pellis-van Berkel, W., Verheijen, M.H., Cuppen, E., Asahina, M., de Rooij, J., Jansen, G., Plasterk, R.H., Bos, J.L., and Zwartkruis, F.J. (2005). Requirement of the Caenorhabditis elegans RapGEF pxf-1 and rap-1 for epithelial integrity. Mol. Biol. Cell 16, 106-116. Abstract Article

Peschard, P., McCarthy, A., Leblanc-Dominguez, V., Yeo, M., Guichard, S., Stamp, G., and Marshall, C.J. (2012). Genetic deletion of RALA and RALB small GTPases reveals redundant functions in development and tumorigenesis. Curr. Biol. 22, 2063-2068. Abstract Article

Peters, E.C., Gossett, A.J., Goldstein, B., Der, C.J., and Reiner, D.J. (2013). Redundant canonical and noncanonical Caenorhabditis elegans p21-activated kinase signaling governs distal tip cell migrations. G3 (Bethesda) 3, $181-195$. Abstract Article

Petit, C.S., Roczniak-Ferguson, A., and Ferguson, S.M. (2013). Recruitment of folliculin to lysosomes supports the amino acid-dependent activation of Rag GTPases. J. Cell Biol. 202, 1107-1122. Abstract Article

Piekny, A.J., and Mains, P.E. (2002). Rho-binding kinase (LET-502) and myosin phosphatase (MEL-11) regulate cytokinesis in the early Caenorhabditis elegans embryo. J. Cell Sci. 115, 2271-2282. Abstract Article

Piekny, A.J., Wissmann, A., and Mains, P.E. (2000). Embryonic morphogenesis in Caenorhabditis elegans integrates the activity of LET-502 Rho-binding kinase, MEL-11 myosin phosphatase, DAF-2 insulin receptor and FEM-2 PP2c phosphatase. Genetics 156, 1671-1689. Abstract Article

Poinat, P., De Arcangelis, A., Sookhareea, S., Zhu, X., Hedgecock, E.M., Labouesse, M., and Georges-Labouesse, E. (2002). A conserved interaction between $\beta 1$ integrin/PAT-3 and Nck-interacting kinase/MIG-15 that mediates commissural axon navigation in C. elegans. Curr. Biol. 12, 622-631. Abstract Article

Poole, E., Groves, I., MacDonald, A., Pang, Y., Alcami, A., and Sinclair, J. (2009). Identification of TRIM23 as a cofactor involved in the regulation of NF- $\mathrm{KB}$ by human cytomegalovirus. J. Virol. 83, 3581-3590. Abstract Article

Poteryaev, D., Datta, S., Ackema, K., Zerial, M., and Spang, A. (2010). Identification of the switch in early-to-late endosome transition. Cell 141, 497-508. Abstract Article

Poteryaev, D., Fares, H., Bowerman, B., and Spang, A. (2007). Caenorhabditis elegans SAND-1 is essential for RAB-7 function in endosomal traffic. EMBO J. 26, 301-312. Abstract Article

Poteryaev, D., Squirrell, J.M., Campbell, J.M., White, J.G., and Spang, A. (2005). Involvement of the actin cytoskeleton and homotypic membrane fusion in ER dynamics in Caenorhabditis elegans. Mol. Biol. Cell 16, 2139-2153. Abstract Article 
Poulin, G., and Ahringer, J. (2005). Living on the edge. Genome Biol. 6, 307. Abstract Article

Prior, I.A., and Hancock, J.F. (2012). Ras trafficking, localization and compartmentalized signalling. Semin. Cell Dev. Biol. 23, 145-153. Abstract Article

Puschel, A.W. (2007). GTPases in semaphorin signaling. Adv. Exp. Med. Biol. 600, 12-23. Abstract Article

Pylayeva-Gupta, Y., Grabocka, E., and Bar-Sagi, D. (2011). RAS oncogenes: weaving a tumorigenic web. Nature Rev. Cancer 11, 761-774. Abstract Article

Quinn, C.C., and Wadsworth, W.G. (2008). Axon guidance: asymmetric signaling orients polarized outgrowth. Trends Cell Biol. 18, 597-603. Abstract Article

Raaijmakers, J.H., and Bos, J.L. (2009). Specificity in Ras and Rap signaling. J. Biol. Chem. 284, $10995-10999$. Abstract Article

Ratner, N., and Miller, S.J. (2015). A RASopathy gene commonly mutated in cancer: the neurofibromatosis type 1 tumour suppressor. Nature Rev. Cancer 15, 290-301. Abstract Article

Rauen, K.A. (2013). The RASopathies. Annu. Rev. Genomics Hum. Genet. 14, 355-369. Abstract Article

Reddien, P.W., and Horvitz, H.R. (2000). CED-2/CrkII and CED-10/Rac control phagocytosis and cell migration in Caenorhabditis elegans. Nat. Cell Biol. 2, 131-136. Abstract Article

Rehmann, H., Bruning, M., Berghaus, C., Schwarten, M., Kohler, K., Stocker, H., Stoll, R., Zwartkruis, F.J., and Wittinghofer, A. (2008). Biochemical characterisation of TCTP questions its function as a guanine nucleotide exchange factor for Rheb. FEBS Lett. 582, 3005-3010. Abstract Article

Reiner, D.J. (2011). Ras effector switching as a developmental strategy. Small GTPases 2, 109-112. Abstract Article

Reinstein, J., Schlichting, I., Frech, M., Goody, R.S., and Wittinghofer, A. (1991). p21 with a phenylalanine $28 \rightarrow$ leucine mutation reacts normally with the GTPase activating protein GAP but nevertheless has transforming properties. J. Biol. Chem. 266, 17700-17706. Abstract Article

Repasky, G.A., Chenette, E.J., and Der, C.J. (2004). Renewing the conspiracy theory debate: does Raf function alone to mediate Ras oncogenesis? Trends Cell Biol. 14, 639-647. Abstract Article

Ridley, A.J., and Hall, A. (1992). The small GTP-binding protein rho regulates the assembly of focal adhesions and actin stress fibers in response to growth factors. Cell 70, 389-399. Abstract Article

Ridley, A.J., Paterson, H.F., Johnston, C.L., Diekmann, D., and Hall, A. (1992). The small GTP-binding protein rac regulates growth factor-induced membrane ruffling. Cell 70, 401-410. Abstract Article

Robinson, M.S. (2004). Adaptable adaptors for coated vesicles. Trends Cell Biol. 14, 167-174. Abstract Article

Rocheleau, C.E., Howard, R.M., Goldman, A.P., Volk, M.L., Girard, L.J., and Sundaram, M.V. (2002). A lin-45 raf enhancer screen identifies eor-1, eor-2 and unusual alleles of Ras pathway genes in Caenorhabditis elegans. Genetics 161, 121-131. Abstract Article

Rodriguez-Viciana, P., Oses-Prieto, J., Burlingame, A., Fried, M., and McCormick, F. (2006). A phosphatase holoenzyme comprised of Shoc2/Sur8 and the catalytic subunit of PP1 functions as an M-Ras effector to modulate Raf activity. Mol. Cell 22, 217-230. Abstract Article

Rodriguez-Viciana, P., Sabatier, C., and McCormick, F. (2004). Signaling specificity by Ras family GTPases is determined by the full spectrum of effectors they regulate. Mol. Cell. Biol. 24, 4943-4954. Abstract Article

Rossman, K.L., Der, C.J., and Sondek, J. (2005). GEF means go: turning on RHO GTPases with guanine nucleotide-exchange factors. Nat. Rev. Mol. Cell Biol. 6, 167-180. Abstract Article 
Rozakis-Adcock, M., McGlade, J., Mbamalu, G., Pelicci, G., Daly, R., Li, W., Batzer, A., Thomas, S., Brugge, J., Pelicci, P.G., et al. (1992). Association of the Shc and Grb2/Sem5 SH2-containing proteins is implicated in activation of the Ras pathway by tyrosine kinases. Nature 360,689-692. Abstract Article

Sablina, A.A., Chen, W., Arroyo, J.D., Corral, L., Hector, M., Bulmer, S.E., DeCaprio, J.A., and Hahn, W.C. (2007). The tumor suppressor PP2A A $\beta$ regulates the RalA GTPase. Cell 129, 969-982. Abstract Article

Saleh, M.C., van Rij, R.P., Hekele, A., Gillis, A., Foley, E., O'Farrell, P.H., and Andino, R. (2006). The endocytic pathway mediates cell entry of dsRNA to induce RNAi silencing. Nat. Cell Biol. 8, 793-802. Abstract Article

Samuelson, A.V., Carr, C.E., and Ruvkun, G. (2007). Gene activities that mediate increased life span of C. elegans insulin-like signaling mutants. Genes Dev. 21, 2976-2994. Abstract Article

Sancak, Y., Peterson, T.R., Shaul, Y.D., Lindquist, R.A., Thoreen, C.C., Bar-Peled, L., and Sabatini, D.M. (2008). The Rag GTPases bind raptor and mediate amino acid signaling to mTORC1. Science 320, 1496-1501. Abstract Article

Sann, S.B., Crane, M.M., Lu, H., and Jin, Y. (2012). rabx-5 regulates RAB-5 early endosomal compartments and synaptic vesicles in C. elegans. PLoS One 7, e37930. Abstract Article

Sasaki, A., Nakae, I., Nagasawa, M., Hashimoto, K., Abe, F., Saito, K., Fukuyama, M., Gengyo-Ando, K., Mitani, S., Katada, T., et al. (2013). Arl8/ARL-8 functions in apoptotic cell removal by mediating phagolysosome formation in Caenorhabditis elegans. Mol. Biol. Cell 24, 1584-1592. Abstract Article

Sasidharan, N., Sumakovic, M., Hannemann, M., Hegermann, J., Liewald, J.F., Olendrowitz, C., Koenig, S., Grant, B.D., Rizzoli, S.O., Gottschalk, A., et al. (2012). RAB-5 and RAB-10 cooperate to regulate neuropeptide release in Caenorhabditis elegans. Proc. Natl. Acad. Sci. U. S. A. 109, 18944-18949. Abstract Article

Sato, K., Norris, A., Sato, M., and Grant, B.D. C. elegans as a model for membrane traffic (April 25, 2014), WormBook, ed. The C. elegans Research Community, WormBook, doi/10.1895/wormbook.1.77.2, http://www.wormbook.org. Abstract Article

Sato, K., Sato, M., Audhya, A., Oegema, K., Schweinsberg, P., and Grant, B.D. (2006). Dynamic regulation of caveolin-1 trafficking in the germ line and embryo of Caenorhabditis elegans. Mol. Biol. Cell 17, $3085-3094$. Abstract Article

Sato, M., Grant, B.D., Harada, A., and Sato, K. (2008). Rab11 is required for synchronous secretion of chondroitin proteoglycans after fertilization in Caenorhabditis elegans. J. Cell Sci. 121, 3177-3186. Abstract Article

Sato, M., Sato, K., Fonarev, P., Huang, C.J., Liou, W., and Grant, B.D. (2005). Caenorhabditis elegans RME-6 is a novel regulator of RAB-5 at the clathrin-coated pit. Nat. Cell Biol. 7, 559-569. Abstract Article

Sato, M., Sato, K., Liou, W., Pant, S., Harada, A., and Grant, B.D. (2008). Regulation of endocytic recycling by $C$. elegans Rab35 and its regulator RME-4, a coated-pit protein. EMBO J. 27, 1183-1196. Abstract Article

Schmick, M., Kraemer, A., and Bastiaens, P.I. (2015). Ras moves to stay in place. Trends Cell Biol. 25, $190-197$. Abstract Article

Schmutz, C., Stevens, J., and Spang, A. (2007). Functions of the novel RhoGAP proteins RGA-3 and RGA-4 in the germ line and in the early embryo of C. elegans. Development 134, 3495-3505. Abstract Article

Schonegg, S., Constantinescu, A.T., Hoege, C., and Hyman, A.A. (2007). The Rho GTPase-activating proteins RGA-3 and RGA-4 are required to set the initial size of PAR domains in Caenorhabditis elegans one-cell embryos.

Proc. Natl. Acad. Sci. U. S. A. 104, 14976-14981. Abstract Article

Schonegg, S., and Hyman, A.A. (2006). CDC-42 and RHO-1 coordinate acto-myosin contractility and PAR protein localization during polarity establishment in C. elegans embryos. Development 133, 3507-3516. Abstract Article

Schreiber, M.A., Pierce-Shimomura, J.T., Chan, S., Parry, D., and McIntire, S.L. (2010). Manipulation of behavioral decline in Caenorhabditis elegans with the Rag GTPase raga-1. PLoS Genet. 6, e1000972. Abstract Article 
Schutzman, J.L., Borland, C.Z., Newman, J.C., Robinson, M.K., Kokel, M., and Stern, M.J. (2001). The Caenorhabditis elegans EGL-15 signaling pathway implicates a DOS-like multisubstrate adaptor protein in fibroblast growth factor signal transduction. Mol. Cell. Biol. 21, 8104-8116. Abstract Article

Selfors, L.M., Schutzman, J.L., Borland, C.Z., and Stern, M.J. (1998). soc-2 encodes a leucine-rich repeat protein implicated in fibroblast growth factor receptor signaling. Proc. Natl. Acad. Sci. U. S. A. 95, 6903-6908. Abstract Article

Seydoux, G., and Greenwald, I. (1989). Cell autonomy of lin-12 function in a cell fate decision in C. elegans. Cell 57, 1237-1245. Abstract Article

Shakir, M.A., Gill, J.S., and Lundquist, E.A. (2006). Interactions of UNC-34 Enabled with Rac GTPases and the NIK kinase MIG-15 in Caenorhabditis elegans axon pathfinding and neuronal migration. Genetics 172, 893-913. Abstract Article

Shakir, M.A., Jiang, K., Struckhoff, E.C., Demarco, R.S., Patel, F.B., Soto, M.C., and Lundquist, E.A. (2008). The Arp2/3 activators WAVE and WASP have distinct genetic interactions With Rac GTPases in Caenorhabditis elegans axon guidance. Genetics 179, 1957-1971. Abstract Article

Shaye, D.D., and Greenwald, I. (2002). Endocytosis-mediated downregulation of LIN-12/Notch upon Ras activation in Caenorhabditis elegans. Nature 420,686-690. Abstract Article

Shaye, D.D., and Greenwald, I. (2005). LIN-12/Notch trafficking and regulation of DSL ligand activity during vulval induction in Caenorhabditis elegans. Development 132, 5081-5092. Abstract Article

Sherwood, D.R., Butler, J.A., Kramer, J.M., and Sternberg, P.W. (2005). FOS-1 promotes basement-membrane removal during anchor-cell invasion in C. elegans. Cell 121, 951-962. Abstract Article

Shi, A., Chen, C.C., Banerjee, R., Glodowski, D., Audhya, A., Rongo, C., and Grant, B.D. (2010). EHBP-1 functions with RAB-10 during endocytic recycling in Caenorhabditis elegans. Mol. Biol. Cell 21, $2930-2943$. Abstract Article

Shi, A., and Grant, B.D. (2013). Interactions between Rab and Arf GTPases regulate endosomal phosphatidylinositol-4,5-bisphosphate during endocytic recycling. Small GTPases 4, 106-109. Abstract Article

Shi, A., Liu, O., Koenig, S., Banerjee, R., Chen, C.C., Eimer, S., and Grant, B.D. (2012). RAB-10-GTPase-mediated regulation of endosomal phosphatidylinositol-4,5-bisphosphate. Proc. Natl. Acad. Sci. U. S. A. 109, E2306-2315. Abstract Article

Shibatohge, M., Kariya, K., Liao, Y., Hu, C.D., Watari, Y., Goshima, M., Shima, F., and Kataoka, T. (1998). Identification of PLC210, a Caenorhabditis elegans phospholipase C, as a putative effector of Ras. J. Biol. Chem. 273, 6218-6222. Abstract Article

Shim, J., Sternberg, P.W., and Lee, J. (2000). Distinct and redundant functions of $\mu 1$ medium chains of the AP-1 clathrin-associated protein complex in the nematode Caenorhabditis elegans. Mol. Biol. Cell 11, $2743-2756$. Abstract Article

Shirakawa, R., Fukai, S., Kawato, M., Higashi, T., Kondo, H., Ikeda, T., Nakayama, E., Okawa, K., Nureki, O., Kimura, T., et al. (2009). Tuberous sclerosis tumor suppressor complex-like complexes act as GTPase-activating proteins for Ral GTPases. J. Biol. Chem. 284, 21580-21588. Abstract Article

Shirakawa, R., and Horiuchi, H. (2015). Ral GTPases: crucial mediators of exocytosis and tumourigenesis. J. Biochem. 157, 285-299. Abstract Article

Shutes, A., Berzat, A.C., Cox, A.D., and Der, C.J. (2004). Atypical mechanism of regulation of the Wrch-1 Rho family small GTPase. Curr. Biol. 14, 2052-2056. Abstract Article

Sieburth, D., Ch'ng, Q., Dybbs, M., Tavazoie, M., Kennedy, S., Wang, D., Dupuy, D., Rual, J.F., Hill, D.E., Vidal, M., et al. (2005). Systematic analysis of genes required for synapse structure and function. Nature 436, $510-517$. Abstract Article 
Sieburth, D.S., Sun, Q., and Han, M. (1998). SUR-8, a conserved Ras-binding protein with leucine-rich repeats, positively regulates Ras-mediated signaling in C. elegans. Cell 94, 119-130. Abstract Article

Simmer, F., Moorman, C., van der Linden, A.M., Kuijk, E., van den Berghe, P.V., Kamath, R.S., Fraser, A.G., Ahringer, J., and Plasterk, R.H. (2003). Genome-wide RNAi of C. elegans using the hypersensitive rrf-3 strain reveals novel gene functions. PLoS Biol. 1, E12. Abstract Article

Simske, J.S., and Hardin, J. (2001). Getting into shape: epidermal morphogenesis in Caenorhabditis elegans embryos. Bioessays 23, 12-23. Abstract Article

Simske, J.S., Kaech, S.M., Harp, S.A., and Kim, S.K. (1996). LET-23 receptor localization by the cell junction protein LIN-7 during C. elegans vulval induction. Cell 85, 195-204. Abstract Article

Simske, J.S., and Kim, S.K. (1995). Sequential signalling during Caenorhabditis elegans vulval induction. Nature 375, 142-146. Abstract Article

Singhvi, A., Teuliere, J., Talavera, K., Cordes, S., Ou, G., Vale, R.D., Prasad, B.C., Clark, S.G., and Garriga, G. (2011). The Arf GAP CNT-2 regulates the apoptotic fate in C. elegans asymmetric neuroblast divisions. Curr. Biol. 21, 948-954. Abstract Article

Skop, A.R., Liu, H., Yates, J., 3rd, Meyer, B.J., and Heald, R. (2004). Dissection of the mammalian midbody proteome reveals conserved cytokinesis mechanisms. Science 305, 61-66. Abstract Article

Skorobogata, O., Escobar-Restrepo, J.M., and Rocheleau, C.E. (2014). An AGEF-1/Arf GTPase/AP-1 ensemble antagonizes LET-23 EGFR basolateral localization and signaling during $C$. elegans vulva induction. PLoS Genet. 10, e1004728. Abstract Article

Skorobogata, O., and Rocheleau, C.E. (2012). RAB-7 antagonizes LET-23 EGFR signaling during vulva development in Caenorhabditis elegans. PLoS One 7, e36489. Abstract Article

Soto, M.C., Qadota, H., Kasuya, K., Inoue, M., Tsuboi, D., Mello, C.C., and Kaibuchi, K. (2002). The GEX-2 and GEX-3 proteins are required for tissue morphogenesis and cell migrations in C. elegans. Genes Dev. 16, 620-632. Abstract Article

Soukas, A.A., Kane, E.A., Carr, C.E., Melo, J.A., and Ruvkun, G. (2009). Rictor/TORC2 regulates fat metabolism, feeding, growth, and life span in Caenorhabditis elegans. Genes Dev. 23, 496-511. Abstract Article

Spencer, A.G., Orita, S., Malone, C.J., and Han, M. (2001). A RHO GTPase-mediated pathway is required during P cell migration in Caenorhabditis elegans. Proc. Natl. Acad. Sci. U. S. A. 98, 13132-13137. Abstract Article

Spiga, F.M., Prouteau, M., and Gotta, M. (2013). The TAO kinase KIN-18 regulates contractility and establishment of polarity in the C. elegans embryo. Dev. Biol. 373, 26-38. Abstract Article

Stamnes, M.A., and Rothman, J.E. (1993). The binding of AP-1 clathrin adaptor particles to Golgi membranes requires ADP-ribosylation factor, a small GTP-binding protein. Cell 73, 999-1005. Abstract Article

Stavoe, A.K., and Colon-Ramos, D.A. (2012). Netrin instructs synaptic vesicle clustering through Rac GTPase, MIG-10, and the actin cytoskeleton. J. Cell Biol. 197, 75-88. Abstract Article

Sternberg, P.W. (1988). Lateral inhibition during vulval induction in Caenorhabditis elegans. Nature 335, 551-554. Abstract Article

Sternberg, P.W., Vulval development (June, 25 2005), WormBook, ed. The C. elegans Research Community, WormBook, doi/10.1895/wormbook.1.6.1, http://www.wormbook.org. Abstract Article

Sternberg, P.W., and Horvitz, H.R. (1986). Pattern formation during vulval development in C. elegans. Cell 44, 761-772. Abstract Article

Sternberg, P.W., and Horvitz, H.R. (1989). The combined action of two intercellular signaling pathways specifies three cell fates during vulval induction in C. elegans. Cell 58, 679-693. Abstract Article 
Stetak, A., Gutierrez, P., and Hajnal, A. (2008). Tissue-specific functions of the Caenorhabditis elegans p120 Ras GTPase activating protein GAP-3. Dev. Biol. 323, 166-176. Abstract Article

Steven, R., Kubiseski, T.J., Zheng, H., Kulkarni, S., Mancillas, J., Ruiz Morales, A., Hogue, C.W., Pawson, T., and Culotti, J. (1998). UNC-73 activates the Rac GTPase and is required for cell and growth cone migrations in $C$. elegans. Cell 92, 785-795. Abstract Article

Steven, R., Zhang, L., Culotti, J., and Pawson, T. (2005). The UNC-73/Trio RhoGEF-2 domain is required in separate isoforms for the regulation of pharynx pumping and normal neurotransmission in C. elegans. Genes Dev. 19, 2016-2029. Abstract Article

Straud, S., Lee, I., Song, B., Avery, L., and You, Y.J. (2013). The jaw of the worm: GTPase-activating protein EAT-17 regulates grinder formation in Caenorhabditis elegans. Genetics 195, 115-125. Abstract Article

Struckhoff, E.C., and Lundquist, E.A. (2003). The actin-binding protein UNC-115 is an effector of Rac signaling during axon pathfinding in C. elegans. Development 130,693-704. Abstract Article

Su, M., Merz, D.C., Killeen, M.T., Zhou, Y., Zheng, H., Kramer, J.M., Hedgecock, E.M., and Culotti, J.G. (2000). Regulation of the UNC-5 netrin receptor initiates the first reorientation of migrating distal tip cells in Caenorhabditis elegans. Development 127, 585-594. Abstract Article

Sulston, J.E., Schierenberg, E., White, J.G., and Thomson, J.N. (1983). The embryonic cell lineage of the nematode Caenorhabditis elegans. Dev. Biol. 100, 64-119. Abstract Article

Sumakovic, M., Hegermann, J., Luo, L., Husson, S.J., Schwarze, K., Olendrowitz, C., Schoofs, L., Richmond, J., and Eimer, S. (2009). UNC-108/RAB-2 and its effector RIC-19 are involved in dense core vesicle maturation in Caenorhabditis elegans. J. Cell Biol. 186, 897-914. Abstract Article

Sun, J., Qian, Y., Hamilton, A.D., and Sebti, S.M. (1998). Both farnesyltransferase and geranylgeranyltransferase I inhibitors are required for inhibition of oncogenic K-Ras prenylation but each alone is sufficient to suppress human tumor growth in nude mouse xenografts. Oncogene 16, 1467-1473. Abstract Article

Sun, L., Liu, O., Desai, J., Karbassi, F., Sylvain, M.A., Shi, A., Zhou, Z., Rocheleau, C.E., and Grant, B.D. (2012). CED-10/Rac1 regulates endocytic recycling through the RAB-5 GAP TBC-2. PLoS Genet. 8, e1002785. Abstract Article

Sundaram, M., Yochem, J., and Han, M. (1996). A Ras-mediated signal transduction pathway is involved in the control of sex myoblast migration in Caenorhabditis elegans. Development 122, 2823-2833. Abstract Article

Sundaram M.V. Canonical RTK-Ras-ERK signaling and related alternative pathways (July 1, 2013), WormBook, ed. The C. elegans Research Community, WormBook, doi/10.1895/wormbook.1.80.2, http:/www.wormbook.org. Abstract Article

Tada, M., Gengyo-Ando, K., Kobayashi, T., Fukuyama, M., Mitani, S., Kontani, K., and Katada, T. (2012). Neuronally expressed Ras-family GTPase Di-Ras modulates synaptic activity in Caenorhabditis elegans. Genes Cells 17, 778-789. Abstract Article

Takenaka, M., Inoue, H., Takeshima, A., Kakura, T., and Hori, T. (2013). C. elegans Rassf homolog, rasf-1, is functionally associated with rab-39 Rab GTPase in oxidative stress response. Genes Cells 18, 203-210. Abstract Article

Tanaka, D., Kameyama, K., Okamoto, H., and Doi, M. (2008). Caenorhabditis elegans Rab escort protein (REP-1) differently regulates each Rab protein function and localization in a tissue-dependent manner. Genes Cells 13 , 1141-1157. Abstract

Tannoury, H., Rodriguez, V., Kovacevic, I., Ibourk, M., Lee, M., and Cram, E.J. (2010). CACN-1/Cactin interacts genetically with MIG-2 GTPase signaling to control distal tip cell migration in C. elegans. Dev. Biol. 341, $176-185$. Abstract Article 
Tao, W., Pennica, D., Xu, L., Kalejta, R.F., and Levine, A.J. (2001). Wrch-1, a novel member of the Rho gene family that is regulated by Wnt-1. Genes Dev. 15, 1796-1807. Abstract Article

Tay, Y., Rinn, J., and Pandolfi, P.P. (2014). The multilayered complexity of ceRNA crosstalk and competition. Nature 505, 344-352. Abstract Article

Teuliere, J., Cordes, S., Singhvi, A., Talavera, K., and Garriga, G. (2014). Asymmetric neuroblast divisions producing apoptotic cells require the cytohesin GRP-1 in Caenorhabditis elegans. Genetics 198, 229-247. Abstract Article

Thapliyal, A., Verma, R., and Kumar, N. (2014). Small G proteins Dexras1 and RHES and their role in pathophysiological processes. Int. J. Cell Biol. 2014, 308535. Abstract Article

Thompson, O., Edgley, M., Strasbourger, P., Flibotte, S., Ewing, B., Adair, R., Au, V., Chaudhry, I., Fernando, L., Hutter, H., et al. (2013). The million mutation project: a new approach to genetics in Caenorhabditis elegans. Genome Res. 23, 1749-1762. Abstract Article

Traub, L.M., Ostrom, J.A., and Kornfeld, S. (1993). Biochemical dissection of AP-1 recruitment onto Golgi membranes. J. Cell Biol. 123, 561-573. Abstract Article

Tsun, Z.Y., Bar-Peled, L., Chantranupong, L., Zoncu, R., Wang, T., Kim, C., Spooner, E., and Sabatini, D.M. (2013). The folliculin tumor suppressor is a GAP for the RagC/D GTPases that signal amino acid levels to mTORC1. Mol. Cell 52, 495-505. Abstract Article

Updike, D.L., and Strome, S. (2009). A genomewide RNAi screen for genes that affect the stability, distribution and function of P granules in Caenorhabditis elegans. Genetics 183, 1397-1419. Abstract Article

Valencia, A., Chardin, P., Wittinghofer, A., and Sander, C. (1991). The ras protein family: evolutionary tree and role of conserved amino acids. Biochemistry 30, 4637-4648. Abstract Article

van Dam, T.J., Zwartkruis, F.J., Bos, J.L., and Snel, B. (2011). Evolution of the TOR pathway. J. Mol. Evol. 73, 209-220. Abstract Article

van den Berg, M.C., van Gogh, I.J., Smits, A.M., van Triest, M., Dansen, T.B., Visscher, M., Polderman, P.E., Vliem, M.J., Rehmann, H., and Burgering, B.M. (2013). The small GTPase RALA controls c-Jun N-terminal kinase-mediated FOXO activation by regulation of a JIP1 scaffold complex. J. Biol. Chem. 288, 21729-21741. Abstract Article

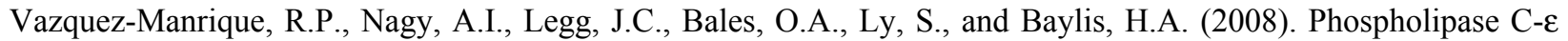
regulates epidermal morphogenesis in Caenorhabditis elegans. PLoS Genet. 4, e1000043. Abstract Article

Vellai, T., Takacs-Vellai, K., Zhang, Y., Kovacs, A.L., Orosz, L., and Muller, F. (2003). Genetics: influence of TOR kinase on lifespan in C. elegans. Nature 426, 620. Abstract Article

Walck-Shannon, E., Reiner, D., and Hardin, J. (2015). Polarized Rac-dependent protrusions drive epithelial intercalation in the embryonic epidermis of C. elegans. Development 142, 3549-3560. Abstract Article

Wandinger-Ness, A., and Zerial, M. (2014). Rab proteins and the compartmentalization of the endosomal system. Cold Spring Harb. Perspect. Biol. 6, a022616. Abstract Article

Wang, X., Fonseca, B.D., Tang, H., Liu, R., Elia, A., Clemens, M.J., Bommer, U.A., and Proud, C.G. (2008). Re-evaluating the roles of proposed modulators of mammalian target of rapamycin complex 1 (mTORC1) signaling. J. Biol. Chem. 283, 30482-30492. Abstract Article

Wang, Y., He, H., Srivastava, N., Vikarunnessa, S., Chen, Y.B., Jiang, J., Cowan, C.W., and Zhang, X. (2012). Plexins are GTPase-activating proteins for Rap and are activated by induced dimerization. Sci. Signal. 5, ra6. Abstract Article

Wang, Y., Pascoe, H.G., Brautigam, C.A., He, H., and Zhang, X. (2013). Structural basis for activation and non-canonical catalysis of the Rap GTPase activating protein domain of plexin. Elife 2, e01279. Abstract Article

Warburton-Pitt, S.R., Silva, M., Nguyen, K.C., Hall, D.H., and Barr, M.M. (2014). The $n p h p-2$ and arl-13 genetic modules interact to regulate ciliogenesis and ciliary microtubule patterning in C. elegans. PLoS Genet. 10, e1004866. Abstract Article 
Watts, J.L., Etemad-Moghadam, B., Guo, S., Boyd, L., Draper, B.W., Mello, C.C., Priess, J.R., and Kemphues, K.J. (1996). par-6, a gene involved in the establishment of asymmetry in early C. elegans embryos, mediates the asymmetric localization of PAR-3. Development 122, 3133-3140. Abstract Article

Welchman, D.P., Mathies, L.D., and Ahringer, J. (2007). Similar requirements for CDC-42 and the PAR-3/PAR-6/PKC-3 complex in diverse cell types. Dev. Biol. 305, 347-357. Abstract Article

Wennerberg, K., and Der, C.J. (2004). Rho-family GTPases: it's not only Rac and Rho (and I like it). J. Cell Sci. 117, 1301-1312. Abstract Article

Wennerberg, K., Rossman, K.L., and Der, C.J. (2005). The Ras superfamily at a glance. J. Cell Sci. 118, $843-846$. Abstract Article

White, M.A., Nicolette, C., Minden, A., Polverino, A., Van Aelst, L., Karin, M., and Wigler, M.H. (1995). Multiple Ras functions can contribute to mammalian cell transformation. Cell 80, 533-541. Abstract Article

Whitfield, C.W., Benard, C., Barnes, T., Hekimi, S., and Kim, S.K. (1999). Basolateral localization of the Caenorhabditis elegans epidermal growth factor receptor in epithelial cells by the PDZ protein LIN-10. Mol. Biol. Cell 10, 2087-2100. Abstract Article

Williams-Masson, E.M., Malik, A.N., and Hardin, J. (1997). An actin-mediated two-step mechanism is required for ventral enclosure of the C. elegans hypodermis. Development 124, 2889-2901. Abstract Article

Winter, J.F., Hopfner, S., Korn, K., Farnung, B.O., Bradshaw, C.R., Marsico, G., Volkmer, M., Habermann, B., and Zerial, M. (2012). Caenorhabditis elegans screen reveals role of PAR-5 in RAB-11-recycling endosome positioning and apicobasal cell polarity. Nat. Cell Biol. 14, 666-676. Abstract Article

Wissmann, A., Ingles, J., and Mains, P.E. (1999). The Caenorhabditis elegans mel-11 myosin phosphatase regulatory subunit affects tissue contraction in the somatic gonad and the embryonic epidermis and genetically interacts with the Rac signaling pathway. Dev. Biol. 209, 111-127. Abstract Article

Wissmann, A., Ingles, J., McGhee, J.D., and Mains, P.E. (1997). Caenorhabditis elegans LET-502 is related to Rho-binding kinases and human myotonic dystrophy kinase and interacts genetically with a homolog of the regulatory subunit of smooth muscle myosin phosphatase to affect cell shape. Genes Dev. 11, 409-422. Abstract Article

Withee, J., Galligan, B., Hawkins, N., and Garriga, G. (2004). Caenorhabditis elegans WASP and Ena/VASP proteins play compensatory roles in morphogenesis and neuronal cell migration. Genetics $167,1165-1176$. Abstract Article

Wu, B., and Guo, W. (2015). The exocyst at a glance. J. Cell Sci. 128, 2957-2964. Abstract Article

Wu, J.C., Chen, T.Y., Yu, C.T., Tsai, S.J., Hsu, J.M., Tang, M.J., Chou, C.K., Lin, W.J., Yuan, C.J., and Huang, C.Y. (2005). Identification of V23RalA-Ser194 as a critical mediator for Aurora-A-induced cellular motility and transformation by small pool expression screening. J. Biol. Chem. 280, 9013-9022. Abstract Article

Wu, Y.C., Cheng, T.W., Lee, M.C., and Weng, N.Y. (2002). Distinct rac activation pathways control Caenorhabditis elegans cell migration and axon outgrowth. Dev. Biol. 250, 145-155. Abstract Article

Wu, Y.C., and Horvitz, H.R. (1998). C. elegans phagocytosis and cell-migration protein CED-5 is similar to human DOCK180. Nature 392, 501-504. Abstract Article

Wu, Y.E., Huo, L., Maeder, C.I., Feng, W., and Shen, K. (2013). The balance between capture and dissociation of presynaptic proteins controls the spatial distribution of synapses. Neuron 78, 994-1011. Abstract Article

Xu, Y., Taru, H., Jin, Y., and Quinn, C.C. (2015). SYD-1C, UNC-40 (DCC) and SAX-3 (Robo) function interdependently to promote axon guidance by regulating the MIG-2 GTPase. PLoS Genet. 11, e1005185. Abstract Article

Yang, Q., Inoki, K., Kim, E., and Guan, K.L. (2006). TSC1/TSC2 and Rheb have different effects on TORC1 and TORC2 activity. Proc. Natl. Acad. Sci. U. S. A. 103, 6811-6816. Abstract Article

Yang, Y., Lu, J., Rovnak, J., Quackenbush, S.L., and Lundquist, E.A. (2006). SWAN-1, a Caenorhabditis elegans WD repeat protein of the AN11 family, is a negative regulator of Rac GTPase function. Genetics 174, $1917-1932$. Abstract Article 
Yap, S.F., Chen, W., and Lim, L. (1999). Molecular characterization of the Caenorhabditis elegans Rho GDP-dissociation inhibitor. Eur. J. Biochem. 266, 1090-1100. Abstract Article

Yochem, J., Sundaram, M., and Han, M. (1997). Ras is required for a limited number of cell fates and not for general proliferation in Caenorhabditis elegans. Mol. Cell. Biol. 17, 2716-2722. Abstract Article

Yoo, A.S., Bais, C., and Greenwald, I. (2004). Crosstalk between the EGFR and LIN-12/Notch pathways in $C$. elegans vulval development. Science 303, 663-666. Abstract Article

Yoo, A.S., and Greenwald, I. (2005). LIN-12/Notch activation leads to microRNA-mediated down-regulation of Vav in C. elegans. Science 310, 1330-1333. Abstract Article

Young, L.C., Hartig, N., Munoz-Alegre, M., Oses-Prieto, J.A., Durdu, S., Bender, S., Vijayakumar, V., Vietri Rudan, M., Gewinner, C., Henderson, S., et al. (2013). An MRAS, SHOC2, and SCRIB complex coordinates ERK pathway activation with polarity and tumorigenic growth. Mol. Cell 52, 679-692. Abstract Article

Yu, X., Lu, N., and Zhou, Z. (2008). Phagocytic receptor CED-1 initiates a signaling pathway for degrading engulfed apoptotic cells. PLoS Biol. 6, e61. Abstract Article

Zallen, J.A., Peckol, E.L., Tobin, D.M., and Bargmann, C.I. (2000). Neuronal cell shape and neurite initiation are regulated by the Ndr kinase SAX-1, a member of the Orb6/COT-1/warts serine/threonine kinase family. Mol. Biol. Cell 11,3177-3190. Abstract Article

Zand, T.P., Reiner, D.J., and Der, C.J. (2011). Ras effector switching promotes divergent cell fates in C. elegans vulval patterning. Dev. Cell 20,84-96. Abstract Article

Zhang, D., Isack, N.R., Glodowski, D.R., Liu, J., Chen, C.C., Xu, X.Z., Grant, B.D., and Rongo, C. (2012). RAB-6.2 and the retromer regulate glutamate receptor recycling through a retrograde pathway. J. Cell Biol. 196, 85-101. Abstract Article

Zhang, H., Landmann, F., Zahreddine, H., Rodriguez, D., Koch, M., and Labouesse, M. (2011). A tension-induced mechanotransduction pathway promotes epithelial morphogenesis. Nature 471, 99-103. Abstract Article

Zhang, H., Squirrell, J.M., and White, J.G. (2008). RAB-11 permissively regulates spindle alignment by modulating metaphase microtubule dynamics in Caenorhabditis elegans early embryos. Mol. Biol. Cell 19, 2553-2565. Abstract Article

Zhang, Y., Ma, C., Delohery, T., Nasipak, B., Foat, B.C., Bounoutas, A., Bussemaker, H.J., Kim, S.K., and Chalfie, M. (2002). Identification of genes expressed in C. elegans touch receptor neurons. Nature 418, 331-335. Abstract Article

Zhang, Y., and Maduzia, L.L. (2010). Mutations in Caenorhabditis elegans eIF2 $\beta$ permit translation initiation from non-AUG start codons. Genetics 185, 141-152. Abstract Article

Zhou, Y., Wong, C.O., Cho, K.J., van der Hoeven, D., Liang, H., Thakur, D.P., Luo, J., Babic, M., Zinsmaier, K.E., Zhu, M.X., et al. (2015). Membrane potential modulates plasma membrane phospholipid dynamics and K-Ras signaling. Science 349, 873-876. Abstract Article

Zhou, Z., Caron, E., Hartwieg, E., Hall, A., and Horvitz, H.R. (2001). The C. elegans PH domain protein CED-12 regulates cytoskeletal reorganization via a Rho/Rac GTPase signaling pathway. Dev. Cell 1, 477-489. Abstract Article

Zhu, H., Shen, H., Sewell, A.K., Kniazeva, M., and Han, M. (2013). A novel sphingolipid-TORC1 pathway critically promotes postembryonic development in Caenorhabditis elegans. eLife 2, e00429. Abstract Article

Zipkin, I.D., Kindt, R.M., and Kenyon, C.J. (1997). Role of a new Rho family member in cell migration and axon guidance in C. elegans. Cell 90, 883-894. Abstract Article

All WormBook content, except where otherwise noted, is licensed under a Creative

Commons Attribution License. 